\title{
POSITIVE GAUSSIAN KERNELS ALSO HAVE GAUSSIAN MINIMIZERS
}

\author{
FRANCK BARTHE AND PAWE€ WOLFF
}

\begin{abstract}
We study lower bounds on multilinear operators with Gaussian kernels acting on Lebesgue spaces, with exponents below one. We put forward natural conditions when the optimal constant can be computed by inspecting centered Gaussian functions only, and we give necessary and sufficient conditions for this constant to be positive. Our work provides a counterpart to Lieb's results on maximizers of multilinear operators with real Gaussian kernels, also known as the multidimensional Brascamp-Lieb inequality. It unifies and extends several inverse inequalities.
\end{abstract}

\section{INTRODUCTION}

1.1. Background and motivation. Our title hints at Lieb's article "Gaussian kernels have only Gaussian maximizers" [33]. This remarkable work studies operators with Gaussian kernels between Lebesgue spaces $L^{p}\left(\mathbb{R}^{n}, \mathbb{C}\right)$, with norm $\|f\|_{p}=\left(\int_{\mathbb{R}^{n}}|f(y)|^{p} d y\right)^{1 / p}$. These operators are of the following form: for any integrable $f: \mathbb{R}^{n} \rightarrow \mathbb{C}$,

$$
G f(x)=\int_{\mathbb{R}^{n}} e^{-\mathcal{Q}(x, y)} f(y) d y, \quad x \in \mathbb{R}^{m},
$$

where $\mathcal{Q}: \mathbb{R}^{m} \times \mathbb{R}^{n} \rightarrow \mathbb{C}$ is such that $\operatorname{Re}(\mathcal{Q}): \mathbb{R}^{m} \times \mathbb{R}^{n} \rightarrow \mathbb{R}$ is a semi-definite positive quadratic form and $\operatorname{Im}(\mathcal{Q}): \mathbb{R}^{m} \times \mathbb{R}^{n} \rightarrow \mathbb{R}$ is a quadratic form. Lieb has given conditions which ensure that the operator norm of $G: L^{p}\left(\mathbb{R}^{n}, \mathbb{C}\right) \rightarrow L^{q}\left(\mathbb{R}^{m}, \mathbb{C}\right)$ can be computed by inspecting centered Gaussian functions only, i.e. functions of the form $f(y)=e^{-q(y)}$ where $\operatorname{Re}(q)$ is $q$ positive definite quadratic form and $\operatorname{Im}(q)$ is a quadratic form (when $q$ is real-valued, $f$ is a real-valued Gaussian function). Here is a simplified version of his result:

Theorem 1.1 ([33]). With the above notation, the relationship

$$
\|G\|_{L_{p} \rightarrow L_{q}}=\sup \left\{\frac{\|G f\|_{q}}{\|f\|_{p}} ; g \text { centered Gaussian }\right\}
$$

holds in any of the following cases

- $1<p \leq q<+\infty$,

- $1<p, q<+\infty$ and the Gaussian kernel is real (i.e. $\mathcal{Q}$ is a quadratic form). In this case it is enough to consider real-valued Gaussian functions.

An important step of the proof consists in the study of the non-degenerate case when $\operatorname{Re}(\mathcal{Q})$ is definite positive: the operator is shown to be compact, weak topology arguments yield the existence of maximizers of the ratio $\|G f\|_{q} /\|f\|_{p}$, and a careful study of

2010 Mathematics Subject Classification: 26D15, $47 \mathrm{G} 10$.

This paper is partially based upon work supported by the National Science Foundation under Grant No. DMS-1440140 while the first named author was in residence at the Mathematical Sciences Research Institute in Berkeley, California, during the Fall 2017 semester.

Research of the second named author was partially supported by ANR-11-LABX-0040-CIMI within the program ANR-11-IDEX-0002-02 and by the National Science Center, Poland project number 2015/18/A/ST1/00553. 
the product operator $G \otimes G$ with kernel $e^{-\mathcal{Q}(x, y)} e^{-\mathcal{Q}\left(x^{\prime}, y^{\prime}\right)}$ allows to show that they are Gaussian. For further comparison, let us emphasize that these arguments use Banach space techniques, which only apply when $p \geq 1$.

Lieb's theorem extends and unifies many important analytic results. By considering the kernel $e^{i\langle x, y\rangle}$, it recovers the calculation of the norm of the Fourier transform from $L^{p}$ to $L^{p^{\prime}}$ for $p \in(1,2)$, which was first achieved by Beckner [13]. It also encompasses Nelson's theorem for the Ornstein-Uhlenbeck semigroup:

$$
P_{t} f(x)=\int_{\mathbb{R}^{n}} f\left(e^{-t} x+\sqrt{1-e^{-2 t}} y\right) d \gamma_{n}(x),
$$

where $\gamma_{n}$ is the standard Gaussian measure on $\mathbb{R}^{n}, d \gamma_{n}(x)=(2 \pi)^{-n / 2} \exp \left(-|x|^{2} / 2\right) d x$. Nelson's theorem [37] asserts that this operator is hypercontractive: for $p, q>1$ satisfying $e^{2 t} \geq \frac{q-1}{p-1}$, and every measurable function $f$,

$$
\left\|P_{t} f\right\|_{L^{q}\left(\gamma_{n}\right)} \leq\|f\|_{L^{p}\left(\gamma_{n}\right)} .
$$

Lieb's article also addresses multilinear operators with Gaussian kernels, and features an extension of the latter theorem in the case of real-valued kernels and functions. (From now on we only consider real-valued functions).

Theorem 1.2 ([33]). Let $m \geq 1$ and for $i=1, \ldots m$ let $p_{i} \geq 1$ and let $B_{i}: \mathbb{R}^{n} \rightarrow \mathbb{R}^{n_{i}}$ be a linear surjective map. Let $\mathcal{Q}$ be a semi-definite positive quadratic form on $\mathbb{R}^{n}$. For non-identically zero functions $f_{i} \in L^{p_{i}}\left(\mathbb{R}^{n_{i}}, \mathbb{R}\right)$, let

$$
H\left(f_{1}, \ldots, f_{m}\right)=\frac{\int_{\mathbb{R}^{n}} e^{-\mathcal{Q}(x)} \prod_{i=1}^{m} f_{i}\left(B_{i} x\right) d x}{\prod_{i=1}^{m}\left\|f_{i}\right\|_{p_{i}}} .
$$

Then the supremum of $H$ over all such functions is equal to its supremum over centered Gaussian functions only.

Setting $c_{i}=1 / p_{i}$ and replacing $f_{i}$ with $f_{i}^{c_{i}}$ gives an analogous result for the following functional on integrable functions:

$$
I\left(f_{1}, \ldots, f_{m}\right)=\frac{\int_{\mathbb{R}^{n}} e^{-\mathcal{Q}(x)} \prod_{i=1}^{m} f_{i}\left(B_{i} x\right)^{c_{i}} d x}{\prod_{i=1}^{m}\left(\int_{\mathbb{R}^{n_{i}}} f_{i}\right)^{c_{i}}} .
$$

The above theorem is a far-reaching extension of Hölder's inequalities. In the case when $\mathcal{Q}=0$ and the maps $B_{i}$ are linear forms (i.e. $n_{i}=1$ ), it recovers a celebrated inequality of Brascamp and Lieb [20], which allowed these authors to compute the optimal constants in Young's convolution inequality, independently of Beckner [13]. Indeed using duality

$$
\|f * g\|_{r} \leq C\|f\|_{p}\|g\|_{q}
$$

can be rewritten as

$$
\int_{\mathbb{R}^{2 n}} f(x-y) g(y) h(x) d x d y \leq C\|f\|_{p}\|g\|_{q}\|h\|_{r^{\prime}}
$$

The classical Loomis-Whitney inequality [34 and its extension by Finner [27] are also particular cases of Theorem 1.2. The Brascamp-Lieb inequalities found striking applications in convex geometry thanks to the work of K. Ball, see e.g. [4, 3, 5]. He put forward a situation where the optimal constant is 1 , and could use it in order to derive various sharp upper bounds on volumes of convex sets. The "geometric" Brascamp-Lieb inequality reads as follows: if $u_{1}, \ldots, u_{m}$ are unit vectors in $\mathbb{R}^{n}$ and if $c_{1}, \ldots, c_{m} \geq 0$ verify

$$
\sum_{i=1}^{m} c_{i} u_{i} \otimes u_{i}=\operatorname{Id}_{\mathbb{R}^{n}}
$$


where $u_{i} \otimes u_{i}$ is the orthogonal projection onto $\mathbb{R} u_{i}$ and $\operatorname{Id}_{\mathbb{R}^{n}}$ is the identity of $\mathbb{R}^{n}$, then for all integrable functions $f_{i}: \mathbb{R} \rightarrow \mathbb{R}^{+}$,

$$
\int_{\mathbb{R}^{n}} \prod_{i=1}^{m} f_{i}\left(\left\langle x, u_{i}\right\rangle\right)^{c_{i}} d x \leq \prod_{i=1}^{m}\left(\int_{\mathbb{R}} f_{i}\right)^{c_{i}} .
$$

Observe that when $m=n$ and $\left(u_{i}\right)_{i=1}^{n}$ is an orthonormal basis, the inequality becomes an equality by Fubini's theorem. So, in some sense, the geometric Brascamp-Lieb inequality describes an extremal property of orthonormal bases among sets of vectors which decompose the identity as in (1.1). An extension to functions of several variables appears in [11].

Over the years, several related inverse inequalities appeared in the literature. A first and very simple example is the inverse Hölder inequality (obviously, Hölder's inequalities are a particular case of Lieb's theorem): if $\lambda \geq 1$ and $f, g: \mathbb{R}^{n} \rightarrow \mathbb{R}^{+}$then

$$
\int_{\mathbb{R}^{n}} f^{\lambda} g^{1-\lambda} \geq\left(\int_{\mathbb{R}^{n}} f\right)^{\lambda}\left(\int_{\mathbb{R}^{n}} g\right)^{1-\lambda}
$$

provided $\int g>0$ and with the convention that $0 \cdot \infty=0$. By rearranging the terms, this is easily deduced from the usual inequality. The inverse Hölder inequality can also be rewritten as a sort of duality for the (non-normed) spaces $L^{p}$ when $p \in(-\infty, 0) \cup(0,1)$ : for $f, g: \mathbb{R}^{n} \rightarrow \mathbb{R}^{+}$,

$$
\int_{\mathbb{R}^{n}} f g \geq\|f\|_{p}\|g\|_{p^{\prime}}
$$

where $p^{\prime} \in(-\infty, 0) \cup(0,1)$ is still defined by $p^{-1}+\left(p^{\prime}\right)^{-1}=1$. Since the latter inequality is sharp, it follows that for $f \geq 0$,

$$
\|f\|_{p}=\inf _{g \geq 0} \frac{\int f g}{\|g\|_{p^{\prime}}}
$$

Another instance appears in the article of Brascamp and Lieb [20], where a sharp inverse Young inequality is proved: given $p, q, r \in(0,1]$ with $1+1 / r=1 / p+1 / q$, the best constant $C$ such for all positive functions

$$
\|f * g\|_{r} \geq C\|f\|_{p}\|g\|_{q}
$$

is described, and is achieved by Gaussian functions. Observe that thanks to (1.2), the latter can be rewritten as

$$
\int_{\mathbb{R}^{2 n}} f(x-y) g(y) h(x) d x d y \geq C\|f\|_{p}\|g\|_{q}\|h\|_{r^{\prime}} .
$$

Later on, Borell [18] proved a reverse form of Nelson's hypercontractivity: if $p, q \in$ $(-\infty, 1)$ and $e^{2 t} \geq \frac{1-q}{1-p}$ then for all positive functions $f$ on $\mathbb{R}^{n}$ :

$$
\left\|P_{t} f\right\|_{L^{q}\left(\gamma_{n}\right)} \geq\|f\|_{L^{p}\left(\gamma_{n}\right)} .
$$

This bound shows that the Ornstein-Uhlenbeck semigroup improves the positivity of functions (for $p<0,\|f\|_{p}=1 /\|1 / f\|_{|p|}$ and $q \leq p$ ).

Among the examples of reverse inequalities are the Prékopa-Leindler inequalities [38, 32] for all $\lambda \in(0,1)$ and all $f, g: \mathbb{R}^{n} \rightarrow \mathbb{R}^{+}$,

$$
\int_{\mathbb{R}^{n}}^{*} \sup _{z=\lambda x+(1-\lambda) y} f(x)^{\lambda} g(y)^{1-\lambda} d z \geq\left(\int_{\mathbb{R}^{n}} f\right)^{\lambda}\left(\int_{\mathbb{R}^{n}} g\right)^{1-\lambda},
$$

where the left hand side term is an outer integral and the supremum is over all $(x, y) \in$ $\left(\mathbb{R}^{n}\right)^{2}$ verifying $z=\lambda x+(1-\lambda) y$. This functional version of the Brunn-Minkowski 
inequality is actually a particular case of the more general reverse Brascamp-Lieb inequalities proved by the first-named author [9, 11]. For shortness, we only state the rank one geometric version of the reverse Brascamp-Lieb inequalities (and refer to Section 5 for more details): given unit vectors $u_{1}, \ldots, u_{m}$ in $\mathbb{R}^{n}$ and $c_{1}, \ldots, c_{m} \geq 0$ satisfying the decomposition of identity (1.1), for all integrable functions $f_{i}: \mathbb{R} \rightarrow \mathbb{R}^{+}$, it holds

$$
\int_{\mathbb{R}^{n}}^{*} \sup _{x=\sum_{i} c_{i} \theta_{i} u_{i}} \prod_{i=1}^{m} f_{i}\left(\theta_{i}\right)^{c_{i}} d x \geq \prod_{i=1}^{m}\left(\int_{\mathbb{R}} f_{i}\right)^{c_{i}} .
$$

This inequality allows to derive geometric properties which are dual to the ones that can be proved using the Brascamp-Lieb inequality (which was the motivation of K. Ball's conjecture of the reverse inequality). See [11, 10] for the first results in this direction. Again, centered Gaussian functions achieve (or almost achieve) the optimal constant. The reader may object that the latter inequality seems rather different from the other ones. Nevertheless, the supremum being an $L^{\infty}$ norm can be viewed as a limit of $L^{p}$ norms. Building on this idea, Brascamp and Lieb [20] were able to deduce the Prékopa-Leindler inequality as a limit case of their inverse Young inequality. In the same way, the firstnamed author proved in [6] an extension of the inverse Young inequality which recovers as a limit the geometric reverse Brascamp-Lieb inequalities. See [25] for further results in this direction. Actually, in view of the content of the present paper and of the dual features of their applications, a better name for reverse Brascamp-Lieb inequalities could be dual Brascamp-Lieb inequalities.

It is natural to ask for a general principle that would unify and explain these reverse inequalities. They definitely share some common features: they provide lower bounds on integrals involving products of positive functions in terms of their $L^{p}$ norms, often with $p<1$, and Gaussian functions play a special role. A significant progress in this direction was recently made by Chen, Dafnis and Paouris. One of their main results is stated in probabilistic terms:

Theorem $1.3([25])$. Let $n=n_{1}+n_{2}+\ldots+n_{m}$ be positive integers and $\left(X_{1}, \ldots, X_{m}\right)$ be a Gaussian random vector in $\mathbb{R}^{n}$ (where $X_{i} \in \mathbb{R}^{n_{i}}$ ), with covariance matrix $\Sigma$. For each $i$, let $\Sigma_{i}$ denote the covariance matrix of $X_{i}$ (which is a diagonal block of $\Sigma$ ). Let $p_{1}, \ldots, p_{m} \in \mathbb{R} \backslash\{0\}$ and consider the block diagonal matrix $P=\operatorname{diag}\left(p_{1} \Sigma_{1}, \ldots, p_{m} \Sigma_{m}\right)$. Then for all positive functions $f_{1}, \ldots, f_{m}$,

$$
\begin{aligned}
& \text { if } \Sigma \leq P, \text { then } \mathbb{E}\left(\prod_{i=1}^{m} f_{i}\left(X_{i}\right)\right) \leq \prod_{i=1}^{m} \mathbb{E}\left(f_{i}\left(X_{i}\right)^{p_{i}}\right)^{\frac{1}{p_{i}}}, \\
& \text { if } \Sigma \geq P, \text { then } \mathbb{E}\left(\prod_{i=1}^{m} f_{i}\left(X_{i}\right)\right) \geq \prod_{i=1}^{m} \mathbb{E}\left(f_{i}\left(X_{i}\right)^{p_{i}}\right)^{\frac{1}{p_{i}}},
\end{aligned}
$$

Here the order on matrices is for the cone of semi-definite positive matrices.

The first part of the theorem is actually a direct consequence of the general BrascampLieb inequality. The second part is a very neat reverse inequality. Observe that the condition $\Sigma \geq P$ implies, by restriction to diagonal blocks, that $1 \geq p_{i}$. The functional inequality can be rewritten as a lower bound on a multilinear operator with a generalized Gaussian kernel (i.e. the exponential of a quadratic form, without sign condition). Chen, Dafnis and Paouris use transformations of this inequality by linear changes of variables in order to get more, and doing so they succeed to recover most of the above mentioned reverse inequalities. Nevertheless, their results do not have full generality and involve conditions which are sometimes difficult to check. In the note [12], we have announced a 
general result on the optimal constant in inequalities of the form

$$
\int_{H} e^{-\mathcal{Q}(x)} \prod_{k=1}^{m} f_{k}^{c_{k}}\left(B_{k} x\right) d x \geq C \prod_{k=1}^{m}\left(\int_{H_{k}} f_{k}\right)^{c_{k}}
$$

for all positive functions $f_{i}$. The goal of the present paper is to give a full proof of the results of [12, and to provide an extensive answer to the following questions: when is it possible to calculate the best constant $C$ by considering only Gaussian functions? or only centered Gaussian functions? when is there a non-trivial inequality $(C>0)$ ?

1.2. Notation and main results. Here is a description of the setup of this article. Let $0 \leq m^{+} \leq m$ be integers and $H, H_{1}, \ldots, H_{m}$ be Euclidean spaces endowed with the usual Lebesgue measure. For $k=1, \ldots, m$ let $c_{k}$ be a real number satisfying $c_{i}>0$ for $i \leq m^{+}$ and $c_{j}<0$ for $j>m^{+}$, and let $B_{k}: H \rightarrow H_{k}$ be a surjective linear map. Further, let $\mathcal{Q}: H \rightarrow \mathbb{R}$ be a quadratic form with signature $\left(s^{+}(\mathcal{Q}), s^{-}(\mathcal{Q})\right)$. For measurable functions $f_{k}: H_{k} \rightarrow[0,+\infty]$ satisfying $0<\int_{H_{k}} f_{k}<+\infty$ define

$$
J\left(f_{1}, \ldots, f_{m}\right)=\frac{\int_{H} e^{-\mathcal{Q}(x)} \prod_{k=1}^{m} f_{k}^{c_{k}}\left(B_{k} x\right) d x}{\prod_{k=1}^{m}\left(\int_{H_{k}} f_{k}\right)^{c_{k}}}
$$

and assume the convention $0 \cdot \infty=0$ for the product $\prod_{k=1}^{m} f_{k}^{c_{k}}\left(B_{k} x\right)$.

Our goal is to study the minimization problem for the functional $J$. It turns out that centered Gaussian functions, i.e. the functions of the form $e^{-q(x)}$ for a positive definite quadratic form $q$, play a pivotal role. One of our main results is the following counterpart to Lieb's Theorem 1.2 .

Theorem 1.4. Let $c_{1}, \ldots, c_{m^{+}}>0, c_{m^{+}+1}, \ldots, c_{m}<0$ with $0 \leq m^{+} \leq m$. Assume that the map $x \mapsto\left(B_{1} x, \ldots, B_{m^{+}} x\right)$ from $H$ to $H_{1} \times \cdots \times H_{m^{+}}$is onto and that

$$
\operatorname{dim} H \geq s^{+}(\mathcal{Q})+\operatorname{dim} H_{1}+\cdots+\operatorname{dim} H_{m^{+}} .
$$

Then $\inf J=\inf _{\mathcal{C G}} J$, where the right-hand side stands for the infimum of $J$ over all choices of centered Gaussian functions $g_{k}$.

Hence a Gaussian minimizers principle holds under some hypotheses. It may fail when they are not fulfilled, but this happens only in degenerate situations. The purpose of Section 2 is to give a full picture of these degenerate cases. This is done via a careful inspection of the values of the functional $J$ on centered as well as general Gaussian functions (i.e. non necessarily centered) of the form $e^{-q+\ell}$, where $q$ is a positive definite quadratic form and $\ell$ is a linear form. This allows to put forward a natural and convenient non-degeneracy condition:

$$
\mathcal{Q}_{\mid \bigcap_{i=1}^{m+} \operatorname{ker} B_{i}} \text { is positive definite and } \operatorname{dim} H \geq s^{+}(\mathcal{Q})+\operatorname{dim} H_{1}+\cdots+\operatorname{dim} H_{m^{+}} \text {. }
$$

Section 3 gives a proof of the Gaussian minimizers principle under the above condition (1.4). The main tool is monotone transportation as in the proof of Brascamp-Lieb inequalities of [11], see also [7, 41]. The presence of negative exponents introduces substantial additional difficulties. A crucial technical step is to use a particular decomposition of the quadratic form $\mathcal{Q}$ which is adapted to the geometric structure of the problem, and is inspired by (1.4). One could have tried and follow other techniques which applied to Brascamp-Lieb inequalities, as semigroup interpolation or stochastic representations [24, 15, 19, 8, 14, 31, 25, 36, 30. Nevertheless the transportation technique has the advantage that it does not require any a priori structural study of extremizers.

In Section 4, we establish the analogue in our setting of the geometric Brascamp-Lieb inequality, and show that it is equivalent to the correlation inequality of Chen, Dafnis and 
Paouris presented here as Theorem 1.3. Our structural study allows a better analysis of equality conditions. Note however that their semigroup proof of the geometric inequality is simpler than ours (somehow for the transportation approach the geometric situation is not easier than the general case).

Section 5 presents a dual form of the inverse Brascamp-Lieb inequalities, which can be obtained from the very same proof. A brief summary of the various types of inequalities is provided.

The next sections are devoted to the question of existence of a non-trivial inverse Brascamp-Lieb inequality. In other words, when is it true that inf $J>0$ ? For BrascampLieb inequalities, the analogous question (of boundedness of multilinear Gaussian operators) was settled in the general case by Bennett, Carbery, Christ and Tao [15, 16] after other contributions in the rank one case [11, 24].

Section [ 6stablishes, for fixed geometric data $\left(\mathcal{Q},\left(B_{k}\right)_{k=1}^{m}\right)$, a convexity property of the set of exponents $\left(c_{k}\right)_{k=1}^{m}$ for which inf $J>0$. We call this set the positivity domain of $J$.

Section 7 gives a description of the positivity domain in the rank one case, i.e. when the maps $B_{k}$ are linear forms and when $s^{+}(\mathcal{Q}), s^{-}(\mathcal{Q}) \leq 1$. In this case, the proof is simple and based on explicit calculations on Gaussian functions. The positivity domain is a polyhedral convex cone which we can describe as an intersection of half-spaces or by generating vectors.

Section 8 deals with the general case. We follow the inductive approach of Bennett, Carbery, Christ and Tao [16]. In our setting, the fact that the quadratic form can have positive and negative parts (and thus corresponds to fixing two Gaussian functions instead of one) makes the analysis more delicate. For simplicity, we state here our characterization in the case when no kernel is involved (the general result is formulated as Theorem 8.9).

Theorem 1.5. Let $c_{1}, \ldots, c_{m^{+}}>0, c_{m^{+}+1}, \ldots, c_{m}<0$ with $0 \leq m^{+} \leq m$. Assume that the map $x \mapsto\left(B_{1} x, \ldots, B_{m^{+}} x\right)$ from $H$ to $H_{1} \times \cdots \times H_{m^{+}}$is a bijection. For any integrable functions $f_{k}: H_{k} \rightarrow[0,+\infty]$ with $\int f_{k}>0$, let

$$
J\left(f_{1}, \ldots, f_{m}\right)=\frac{\int_{H} \prod_{k=1}^{m} f_{k}^{c_{k}}\left(B_{k} x\right) d x}{\prod_{k=1}^{m}\left(\int_{H_{k}} f_{k}\right)^{c_{k}}} .
$$

Then inf $J>0$ if and only if the following two conditions are verified:

(1) $\operatorname{dim} H=\sum_{i=1}^{m} c_{k} \operatorname{dim} H_{k}$,

(2) For every linear subspace $V \subset H$ such that $\operatorname{dim} V=\sum_{i=1}^{m^{+}} \operatorname{dim} B_{i} V$, it holds

$$
\operatorname{dim} V \geq \sum_{k=1}^{m} c_{k} \operatorname{dim} B_{k} V .
$$

If $x \mapsto\left(B_{1} x, \ldots, B_{m^{+}} x\right)$ is not surjective then $\min J=0$. If it is surjective but not injective then $\inf J=+\infty$.

Let us emphasize that in the positivity domain, $c_{i} \geq 1$ for $i=1,2, \ldots, m^{+}$(see Proposition 8.13), which means that our results can be stated in terms of $L^{p_{k}}$-spaces with $p_{k}=1 / c_{k} \leq 1$ and possibly negative.

Let us conclude this introduction with some more notation and comments on the setting. In the rest of the paper, we use the notation $\inf _{\mathcal{G}} J$ for the infimum of $J$ on $m$-tuples of Gaussian functions (not necessarily centered).

We could only require the sets $H_{k}$ to be finite dimensional vector spaces equipped with a Lebesgue measure. Euclidean structures are not relevant for our problems, but working in Euclidean spaces is convenient for explicit calculations for Gaussian functions, as quadratic functions are represented by symmetric linear maps. Also Euclidean structures induce a 
canonical choice of Lebesgue measure. In a context of a Euclidean space, we will use $\langle\cdot, \cdot\rangle$ for the inner product, $|\cdot|$ for the Euclidean norm and for a linear map $L$ between Euclidean spaces, $L^{*}$ will stand for its adjoint. We denote by $Q$ a self-adjoint map on $H$ such that for all $x \in H$,

$$
\mathcal{Q}(x)=\pi\langle x, Q x\rangle .
$$

Eventually let us mention that we allow $H_{k}=\{0\}$ and choose by convention the Lebesgue measure to be the Dirac mass at 0 . For such a $k$, the terms involving $f_{k}$ can be canceled out from (1.3).

1.3. Acknowledgments. This work has benefited from discussions on related topics with several colleagues. We would like to thank in particular Jérôme Bertrand, Max Fathi, Piotr Miłoś, Krzysztof Oleszkiewicz, Grigoris Paouris.

\section{WELL-POSEDNESS OF THE MINIMIZATION PROBLEM AND THE MINIMUM VALUE}

We denote by $B_{+}$the linear map $\left(B_{1}, \ldots, B_{m^{+}}\right)$, that is

$$
\begin{aligned}
B_{+}: H & \rightarrow H_{1} \times \cdots \times H_{m^{+}} \\
x & \mapsto\left(B_{1} x, \ldots, B_{m^{+}} x .\right.
\end{aligned}
$$

2.1. A non-degeneracy condition. We put forward a simple condition on the above map $B_{+}$which allows the functional $J$ to vanish.

Lemma 2.1. (i) If the map $B_{+}$from $H$ to $H_{1} \times \cdots \times H_{m^{+}}$is not onto, then $\inf J=$ $\min J=0$.

(ii) Conversely, if the map $B_{+}$is onto, then all $f_{k}: H_{k} \rightarrow[0,+\infty]$ with $0<\int_{H_{k}} f_{k}<$ $+\infty$ one has $J\left(\left(f_{k}\right)\right) \in(0,+\infty]$.

Proof. (i) Consider a point $\left(a_{1}, \ldots, a_{m^{+}}\right) \in H_{1} \times \cdots \times H_{m^{+}}$, so that its Euclidean distance to the range of the non-surjective linear map $B_{+}$is at least $\sqrt{m^{+}}$. If we denote by $B_{H}(x, r)$ the open ball of center $x$ and radius $r$ in $H$, then

$$
\left(B_{H_{1}}\left(a_{1}, 1\right) \times \cdots \times B_{H_{m}^{+}}\left(a_{m^{+}}, 1\right)\right) \cap\left\{\left(B_{1} x, \ldots, B_{m^{+}} x\right) ; x \in H\right\}=\emptyset .
$$

For $1 \leq i \leq m^{+}$consider the function $f_{i}: H_{i} \rightarrow \mathbb{R}^{+}$defined as the characteristic function of $B_{H_{i}}\left(a_{i}, 1\right)$. Then the latter empty intersection ensures that for all $x \in H$,

$$
\prod_{i=1}^{m^{+}} f_{i}^{c_{i}}\left(B_{i} x\right)=0 .
$$

Therefore, for any choice of functions $\left(f_{j}\right)_{j>m^{+}}$, it holds $J\left(f_{1}, \ldots, f_{m}\right)=0$.

(ii) Since $\prod_{i \leq m^{+}} \int_{H_{i}} f_{i}>0$, the measure of points $\left(z_{1}, \ldots, z_{m^{+}}\right) \in H_{1} \times \cdots \times H_{m^{+}}$for which $\prod_{i \leq m^{+}} f_{i}^{c_{i}}\left(z_{i}\right)>0$ is positive. From the hypothesis that $B_{+}$is onto it follows that the measure of

$$
\left\{x \in H ; \prod_{1 \leq i \leq m^{+}} f_{i}^{c_{i}}\left(B_{i} x\right)>0\right\}
$$

is positive. To conclude it is enough to notice that integrability of $f_{j}$ (for $m^{+}<j<m$ ) implies that $\prod_{j=1+m^{+}}^{m} f_{j}^{c_{j}}\left(B_{j} x\right)>0 x$-a.e. in $H$.

As a consequence of the previous lemma, we will often work under the non-degeneracy assumption that $B_{+}$is surjective. 
2.2. Calculations for centered Gaussian functions. Recall the classical formula $\int_{\mathbb{R}} e^{-\pi t^{2}} d t=1$. From this, it follows that for any self-adjoint operator $A$ on $\mathbb{R}^{d}$ (or a $d$-dimensional Euclidean space),

$$
\int_{\mathbb{R}^{d}} e^{-\pi\langle x, A x\rangle} d x= \begin{cases}\operatorname{det}(A)^{-1 / 2} & \text { if } A \text { is positive definite } \\ +\infty & \text { otherwise. }\end{cases}
$$

When $A$ is positive definite, we denote $g_{A}$ the centered Gaussian function defined by

$$
g_{A}(x)=e^{-\pi\langle x, A x\rangle} .
$$

An elementary computation shows that

$$
J\left(g_{A_{1}}, \ldots, g_{A_{m}}\right)= \begin{cases}\left(\frac{\operatorname{det}\left(Q+\sum_{k=1}^{m} c_{k} B_{k}^{*} A_{k} B_{k}\right)}{\prod_{k=1}^{m}\left(\operatorname{det} A_{k}\right)^{c_{k}}}\right)^{-1 / 2} & \text { if }\left(A_{1}, \ldots, A_{m}\right) \in \Lambda, \\ \infty & \text { otherwise }\end{cases}
$$

where the set $\Lambda$ is defined as follows

$\Lambda=\left\{\left(A_{1}, \ldots, A_{m}\right): A_{k}: H_{k} \rightarrow H_{k}\right.$ and $Q+\sum_{k=1}^{m} c_{k} B_{k}^{*} A_{k} B_{k}: H \rightarrow H$ are positive definite $\}$.

Therefore the infimum of $J$ over centered Gaussian functions equals $D^{-1 / 2}$, where

$$
D=\sup \left\{\frac{\operatorname{det}\left(Q+\sum_{k=1}^{m} c_{k} B_{k}^{*} A_{k} B_{k}\right)}{\prod_{k=1}^{m}\left(\operatorname{det} A_{k}\right)^{c_{k}}}:\left(A_{1}, \ldots, A_{m}\right) \in \Lambda\right\}
$$

with the convention that $D=0$ for $\Lambda=\emptyset$ (and thus $D^{-1 / 2}=\infty$ ).

2.3. Ensuring finiteness for some functions. We investigate the existence of non-zero functions for which $J$ takes a finite value. The right setup for the functional $J$ to be non degenerate on centered Gaussian functions is the following condition:

$$
\mathcal{Q} \text { is positive definite on } \operatorname{ker} B_{+} \text {. }
$$

Proposition 2.2. The following assertions are equivalent:

(i) there exist centered Gaussian functions $g_{1}, \ldots, g_{m}$ with $J\left(g_{1}, \ldots, g_{m}\right)<+\infty$,

(ii) $\Lambda \neq \emptyset$,

(iii) $\mathcal{Q}_{\mid \operatorname{ker} B_{+}}$is positive definite.

Proof. The equivalence of $(i)$ and $(i i)$ is a direct consequence of Formula (2.2). Assertion (ii) is equivalent to the existence of positive maps $\left(A_{k}\right)_{k=1}^{m}$ such that $Q+\sum_{k=1}^{m} c_{k} B_{k}^{*} A_{k} B_{k}$ is positive definite. This can be rewritten as

$$
Q+\sum_{i=1}^{m^{+}} c_{i} B_{i}^{*} A_{i} B_{i}>\sum_{j=1+m^{+}}^{m}\left|c_{j}\right| B_{j}^{*} A_{j} B_{j}
$$

Since one may choose the matrices $A_{j}>0$ arbitrarily small, $(i i)$ is equivalent to the existence of positive maps $\left(A_{i}\right)_{i=1}^{m^{+}}$such that

$$
Q+\sum_{i=1}^{m^{+}} c_{i} B_{i}^{*} A_{i} B_{i}>0
$$

Similarly, one may choose each matrix $A_{i}$ as an arbitrarily large multiple of the identity on $H_{i}$. Hence $(i i)$ is equivalent to the existence of $D>0$ such that $Q+\sum_{i=1}^{m^{+}} D B_{i}^{*} B_{i}>0$, or in terms of quadratic forms

$$
x \mapsto\langle x, Q x\rangle+D \sum_{i=1}^{m^{+}}\left|B_{i} x\right|^{2}=\langle x, Q x\rangle+D\left|B_{+} x\right|^{2}
$$


is positive definite. We may conclude thanks to Lemma 2.3 below for $L=B_{+}$.

Lemma 2.3. Let $\mathcal{R}$ be a quadratic form on $\mathbb{R}^{d}$. Let $L: \mathbb{R}^{d} \rightarrow \mathbb{R}^{k}$ be a linear map between Euclidean spaces. Then the following assertions are equivalent:

(i) There exists $D>0$ such that the quadratic form $x \mapsto \mathcal{R}(x)+D|L x|^{2}$ is positive definite,

(ii) $\mathcal{R}_{\mid \operatorname{ker} L}$ is positive definite.

Proof. If $(i)$ holds then for $D$ large enough $x \mapsto \mathcal{R}(x)+D|L x|^{2}$ is positive definite. Hence its restriction to ker $L$ is also positive definite, namely $\mathcal{R}_{\mid \operatorname{ker} L}$ is positive definite.

Next, let us show that $(i i)$ implies $(i)$, by contradiction. If $(i)$ is not true then for every integer $N$ there exists a unit vector $x_{N} \in S^{d-1} \subset \mathbb{R}^{d}$ such that

$$
\mathcal{R}\left(x_{N}\right)+N\left|L x_{N}\right|^{2} \leq 0 .
$$

By compactness of the unit sphere, one can find a converging subsequence $\left(x_{N_{k}}\right)$. Let $x \in S^{d-1}$ denote its limit.

Since $\mathcal{R}\left(x_{N_{k}}\right) \leq-N_{k}\left|L x_{N_{k}}\right|^{2} \leq 0$, passing to the limit gives $\mathcal{R}(x) \leq 0$. Moreover

$$
\left|L x_{N_{k}}\right|^{2} \leq-\frac{\mathcal{R}\left(x_{N_{k}}\right)}{N_{k}} \leq-\frac{\min _{S^{d-1}} \mathcal{R}}{N_{k}}
$$

so by continuity, letting $k$ go to infinity $|L x|=0$. Hence $x \in \operatorname{ker} L \backslash\{0\}$ verifies $\mathcal{R}(x) \leq 0$, meaning that the restriction of $\mathcal{R}$ to $\operatorname{ker} L$ is not positive definite.

Combined with the above proposition, the forthcoming one shows that if the map $B_{+}$ defined in (2.1) is surjective then the following holds: there exists functions for which $J$ is finite if and only if there exists centered Gaussian functions for which $J$ is finite.

Proposition 2.4. Assume that the map $B_{+}$is surjective. If $\mathcal{Q}_{\mid \operatorname{ker} B_{+}}$is not positive definite, then for every functions $f_{k}: H_{k} \rightarrow \mathbb{R}^{+}$with $\int f_{k} \in(0,+\infty)$, the quantity $J\left(\left(f_{k}\right)_{1 \leq k \leq m}\right)$ is $+\infty$.

Proof. Without loss of generality, we consider arbitrary functions $f_{k}$ with $\int f_{k}=1$. By our hypothesis, there exists a unit vector $v \in H$ such that $\langle v, Q v\rangle \leq 0$ and for all $1 \leq i \leq m^{+}$, $B_{i} v=0$. Let $S \subset H$ be any linear complement of $\mathbb{R} v$. Then there is a positive constant $c_{S}$ such that, decomposing each element of $H$ as $x=y+t v$ with $y \in S$ and $t \in \mathbb{R}$

$$
\begin{aligned}
J\left(\left(f_{k}\right)\right) & =c_{S} \int e^{-\pi\left(\langle y, Q y\rangle+2 t\langle Q y, v\rangle+t^{2}\langle v, Q v\rangle\right)} \prod_{1 \leq i \leq m^{+}} f_{i}^{c_{i}}\left(B_{i} y\right) \prod_{m^{+}<j \leq m} f_{j}^{c_{j}}\left(B_{j} y+t B_{j} v\right) d t d y \\
& \geq c_{S} \int_{S} e^{-\pi\langle y, Q y\rangle} \prod_{1 \leq i \leq m^{+}} f_{i}^{c_{i}}\left(B_{i} y\right)\left(\int_{\mathbb{R}} e^{-2 \pi t\langle Q y, v\rangle} \prod_{m^{+}<j \leq m} f_{j}^{c_{j}}\left(B_{j} y+t B_{j} v\right) d t\right) d y
\end{aligned}
$$

Let us prove that $y$-almost everywhere in $S$, the inner integral equals $+\infty$. To do this, we prove that $y$-a.e. in $S$, the non-negative function $t \mapsto \prod_{m^{+}<j \leq m} f_{j}^{c_{j}}\left(B_{j} y+t B_{j} v\right)$ is bounded from below by a positive constant, except maybe on a set of finite Lebesgue measure. Here are the details:

If $m^{+}<j \leq m$ is such that $B_{j} v=0$ then for all $t, f_{j}^{c_{j}}\left(B_{j} y+t B_{j} v\right)=f_{j}^{c_{j}}\left(B_{j} y\right)$. Since $B_{j}: H \rightarrow H_{i}$ is surjective and $B_{j} v=0$ it follows that the restriction of $B_{j}$ to $S$ is also surjective. Since $\int_{H_{j}} f_{j}<+\infty$, we know that $f_{j}<+\infty$ a.e. in $H_{j}$. As the preimage of a Lebesgue negligible set by a linear surjection is also Lebesgue negligible, we deduce that $y$-a.e in $S, f_{j}\left(B_{j} y\right)<+\infty$. Using that $c_{j}$ is negative, we get that $y$-a.e. in $S, f_{j}^{c_{j}}\left(B_{j} y\right)>0$.

If $m^{+}<j \leq m$ is such that $B_{j} v \neq 0$ we proceed differently. First, for each $y$, one can decompose $B_{j} y$ using orthogonal projections as follows

$$
B_{j} y=P_{\left(\mathbb{R} B_{j} v\right)^{\perp}} B_{j} y+P_{\mathbb{R} B_{j} v} B_{j} y=L_{j} y+t_{j}(y) B_{j} v
$$


where $L_{j}=P_{\left(\mathbb{R} B_{j} v\right)^{\perp}} B_{j}$. By translation invariance of Lebesgue's measure

$$
\begin{aligned}
\operatorname{vol}_{1}\left(\left\{t \in \mathbb{R} ; f_{j}^{c_{j}}\left(B_{j} y+t B_{j} v\right) \leq 1\right\}\right) & =\operatorname{vol}_{1}\left(\left\{t \in \mathbb{R} ; f_{j}\left(L_{j} y+\left(t+t_{j}(y)\right) B_{j} v\right) \geq 1\right\}\right) \\
& =\operatorname{vol}_{1}\left(\left\{t \in \mathbb{R} ; f_{j}\left(L_{j} y+t B_{j} v\right) \geq 1\right\}\right) .
\end{aligned}
$$

Next

$$
1=\int_{H_{j}} f_{j}=\left|B_{j} v\right| \int_{\left(\mathbb{R} B_{j} v\right)^{\perp}} \int_{\mathbb{R}} f_{j}\left(z+t B_{j} v\right) d t d z,
$$

hence $z$-a.e. in $\left(\mathbb{R} B_{j} v\right)^{\perp}$, the inner integral is finite and therefore

$$
\operatorname{vol}_{1}\left(\left\{t \in \mathbb{R} ; f_{j}\left(z+t B_{j} v\right) \geq 1\right\}\right)<+\infty .
$$

Since by construction the above map $L_{j}: S \rightarrow\left(\mathbb{R} B_{j} v\right)^{\perp}$ is linear and onto, it follows that $y$-a.e. in $S,\left\{t \in \mathbb{R} ; f_{j}^{c_{j}}\left(B_{j} y+t B_{j} v\right) \leq 1\right\}$ has finite Lebesgue measure.

Putting everything together, we obtain as claimed that $y$-a.e. in $S, t \mapsto \prod_{j>m^{+}} f_{j}^{c_{j}}\left(B_{j} y+\right.$ $\left.t B_{j} v\right)$ is bounded from below by 1 , except for a set of finite Lebesgue measure. Lemma 2.5 below then yields that $y$-a.e. in $S$ the inner integral in the latter expression for $J\left(\left(f_{k}\right)\right)$ is infinite. Consequently

$$
J\left(\left(f_{k}\right)\right) \geq c_{S} \int_{S} e^{-\pi\langle y, Q y\rangle} \prod_{1 \leq i \leq m^{+}} f_{i}^{c_{i}}\left(B_{i} y\right) \times(+\infty) d y .
$$

So $J\left(\left(f_{k}\right)\right)=+\infty$ provided the set of elements $y \in S$ for which $\prod_{i<m^{+}} f_{i}^{c_{i}}\left(B_{i} y\right)>0$ has positive measure (for at least one choice of $S$ ). To this end we use the hypothesis that $B_{+}$ is surjective and Lemma 2.1(ii) to obtain that $J\left(\left(f_{k}\right)\right)>0$, which readily implies that the set of $x \in H$ for which $\prod_{i \leq m^{+}} f_{i}^{c_{i}}\left(B_{i} x\right)>0$ has positive measure. By integrating over the Grassmannian of hyperplanes in $H$ there exists a non-negligible set of hyperplanes $\mathcal{S}$ such that for each $S \in \mathcal{S}$, the set of $y \in S$ for which $\prod_{i \leq m^{+}} f_{i}^{c_{i}}\left(B_{i} y\right)>0$ has positive measure. Since the set of hyperplanes of $H$ containing $v$ is negligible, there must be a hyperplane not containing $v$ in $\mathcal{S}$.

Lemma 2.5. Let $A \subset \mathbb{R}$ be a Borel set. If $A^{c}$ has finite Lebesgue measure then $\int_{A} e^{t} d t=$ $+\infty$

Proof. Assume on the contrary that $\int_{A} e^{t} d t=C<+\infty$. Then for every $N \in \mathbb{N}$, $e^{N} \operatorname{vol}_{1}(A \cap[N, N+1)) \leq C$. Hence $\operatorname{vol}_{1}\left(A^{c} \cap[N, N+1)\right) \geq 1-C e^{-N}$. Summing over $N \in \mathbb{N}$ gives that $A^{c}$ has infinite measure.

2.4. On the effect of translating Gaussian functions and consequences of positivity. In order to explain the relevance of the hypothesis

$$
\operatorname{dim} H \geq s^{+}(\mathcal{Q})+\operatorname{dim} H_{1}+\cdots+\operatorname{dim} H_{m^{+}}
$$

which appears in Theorem 1.4, we study the value of the functional $J$ on non-centered Gaussian functions.

In order to handle the Gaussian kernel $\exp (-\mathcal{Q})$ as two additional (fixed) Gaussian functions (one function corresponding to a positive exponent and the other corresponding to a negative exponent), we will decompose the quadratic form $\mathcal{Q}$ into a positive and negative part. To this end, note the following simple fact:

Lemma 2.6. Let $S: H \rightarrow X$ and $T: H \rightarrow Y$ be linear maps. The map $(S, T): H \rightarrow$ $X \times Y$ is surjective if and only if $S$ and $T$ are surjective and

$$
\operatorname{ker} S+\operatorname{ker} T=H \text {. }
$$

It is a linear isomorphism if and only if $S$ and $T$ are surjective and $\operatorname{ker} S \oplus \operatorname{ker} T=H$. 
Proof. Assume that the map $(S, T)$ is surjective. Then $S$ and $T$ are surjective too. For (2.6), consider any $x \in H$ and we aim to decompose it into $\operatorname{ker} S$ and $\operatorname{ker} T$. By surjectivity of the map $(S, T)$, there exists $y \in H$ such that $(S y, T y)=(S x, 0)$. Therefore $x-y \in \operatorname{ker} S$ and $y \in \operatorname{ker} T$, hence

$$
x=(x-y)+y \in \operatorname{ker} S+\operatorname{ker} T .
$$

For the other implication, take any $x, y \in H$. From the hypothesis (2.6) it follows that there exists $v \in \operatorname{ker} S$ and $w \in \operatorname{ker} T$ such that $x-y=v+w$, i.e.

$$
x-v=y+w .
$$

Denote $z=x-v$. We clearly have

$$
S z=S(x-v)=S x \quad \text { and } \quad T z=T(y+w)=T y,
$$

i.e. $(S, T) z=(S x, T y)$. This means that $(S, T)$ is surjective, since $(S x, S y)$ is arbitrary in $S H \times T H=X \times Y$, by surjectivity of $S$ and $T$.

The second part of the lemma follows from $\operatorname{ker}(S, T)=\operatorname{ker} S \cap \operatorname{ker} T$.

In what follows we consider any decomposition of $\mathcal{Q}$ of the form

$$
\mathcal{Q}(x)=c_{0} \mathcal{Q}_{+}\left(B_{0} x\right)+c_{m+1} \mathcal{Q}_{-}\left(B_{m+1} x\right)
$$

where $c_{0}>0>c_{m-1}$ are real numbers, $B_{0}: H \rightarrow H_{0}$ and $B_{m+1}: H \rightarrow H_{m+1}$ are surjective linear maps onto Euclidean spaces $H_{0}$ and $H_{m+1}$ (respectively), such that the map $\left(B_{0}, B_{m+1}\right)$ is surjective, or equivalently, by Lemma 2.6.

$$
\text { ker } B_{0}+\operatorname{ker} B_{m+1}=H
$$

and $\mathcal{Q}_{+}, \mathcal{Q}_{-}$are positive definite quadratic forms on $H_{0}$ and $H_{m+1}$ (respectively).

The existence of such decomposition is obvious by considering an eigenvalue decomposition of the self-adjoint map $Q$. Then $B_{0}$ can be taken as the orthogonal projection of $H$ onto $H_{0}$ being a subspace spanned by eigenvectors corresponding to positive eigenvalues of $Q$, and similarly $B_{m+1}$. One can take $c_{0}=1$ and $c_{m+1}=-1$. Condition (2.8) follows from orthogonality of $H_{0}$ and $H_{m+1}$ in $H$. Moreover, we clearly have

$$
s^{+}(\mathcal{Q})=\operatorname{dim} H_{0}, \quad s^{-}(\mathcal{Q})=\operatorname{dim} H_{m+1} .
$$

Conversely, any decomposition of $\mathcal{Q}$ as in (2.7) satisfies (2.9). Indeed, by (2.8), one can find a complement subspace $V$ of ker $B_{0}$ in $H$ which satisfies $V \subseteq \operatorname{ker} B_{m+1}$ and hence $\mathcal{Q}$ is positive definite on $V$. This yields $s^{+}(\mathcal{Q}) \geq \operatorname{dim} V=\operatorname{dim} H-\operatorname{dim} \operatorname{ker} B_{0}=\operatorname{dim} H_{0}$. On the other hand, $\mathcal{Q}$ is negative semi-definite on ker $B_{0}$, hence $s^{+}(\mathcal{Q}) \leq \operatorname{dim} H$-dim ker $B_{0}=$ $\operatorname{dim} H_{0}$. The same argument shows the second assertion of (2.9).

The starting point of the forthcoming calculations is that for any self-adjoint map $A$ on $\mathbb{R}^{d}$ and any vector $b \in \mathbb{R}^{d}$,

$$
\int_{\mathbb{R}^{d}} e^{-\pi\langle x, A x\rangle+2 \pi\langle b, x\rangle} d x= \begin{cases}e^{\pi\left\langle A^{-1} b, b\right\rangle} \operatorname{det}(A)^{-1 / 2} & \text { if } A \text { is positive definite } \\ +\infty & \text { otherwise. }\end{cases}
$$

For $k=1, \ldots, m$, let $A_{k}$ be a positive definite map on $H_{k}$. Moreover, let $A_{0}$ be positive definite map $H_{0}$ such that $\mathcal{Q}_{+}(x)=\pi\left\langle x, A_{0} x\right\rangle$, and similarly define $A_{m+1}$ for $\mathcal{Q}_{-}$. With this notation (2.7) becomes

$$
Q=\sum_{k \in\{0, m+1\}} c_{k} B_{k}^{*} A_{k} B_{k} .
$$

For $k=0, \ldots, m+1$ fix any $b_{k} \in H_{k}$. Since the map $\left(B_{0}, B_{m+1}\right)$ is surjective, we can find a vector $b \in H$ such that $B_{0} b=b_{0}$ and $B_{m+1} b=b_{m+1}$. 
We calculate the value of $J$ on the translated Gaussian functions $g_{A_{k}}\left(\cdot+b_{k}\right)$. By translation invariance of Lebesgue's measure, $\int g_{A_{k}}\left(\cdot+b_{k}\right)=\operatorname{det}\left(A_{k}\right)^{-1 / 2}$. In order to introduce a translation also in the Gaussian kernel, we perform a change of variable $y=x+b$ in the integral

$$
\begin{aligned}
J\left(\left(g_{A_{k}}\left(\cdot+b_{k}\right)\right)\right) \prod_{k=1}^{m} \operatorname{det}\left(A_{k}\right)^{-c_{k} / 2} & =\int_{H} e^{-\pi\langle y, Q y\rangle} \prod_{k=1}^{m} g_{A_{k}}^{c_{k}}\left(B_{k} y+b_{k}\right) d y \\
& =\int_{H} e^{-\pi\langle x+b, Q(x+b)\rangle} \prod_{k=1}^{m} g_{A_{k}}^{c_{k}}\left(B_{k} x+B_{k} b+b_{k}\right) d x \\
& =\int_{H} e^{-\pi \sum_{k \in\{0, m+1\}}\left\langle B_{k} x+b_{k}, A_{k}\left(B_{k} x+b_{k}\right)\right\rangle} \prod_{k=1}^{m} g_{A_{k}}^{c_{k}}\left(B_{k} x+B_{k} b+b_{k}\right) d x .
\end{aligned}
$$

Here it is convenient to set $u_{k}=B_{k} b+b_{k}$ for $k=1,2, \ldots, m^{+}$and $u_{k}=b_{k}$ for $k \in\{0, m+1\}$ (for the sake of consistency of notation). Developing all the quadratic terms shows that the latter integral is equal to

$$
\begin{aligned}
& \int_{H} e^{-\pi\left(\sum_{k=0}^{m+1} c_{k}\left\langle A_{k}\left(B_{k} x+u_{k}\right), B_{k} x+u_{k}\right\rangle\right)} d x \\
= & \int_{H} e^{-\pi\left(\langle x, A x\rangle+2\langle x, v\rangle+\sum_{k=0}^{m+1} c_{k}\left\langle A_{k} u_{k}, u_{k}\right\rangle\right)} d x,
\end{aligned}
$$

where we have set $A=\sum_{k=0}^{m+1} c_{k} B_{k}^{*} A_{k} B_{k}$ and $v=\sum_{k=0}^{m+1} c_{k} B_{k}^{*} A_{k} u_{k}$. From the above calculations and (2.10) it follows that

$$
\left.J\left(\left(g_{A_{k}}\left(\cdot+b_{k}\right)\right)\right)<+\infty \Longleftrightarrow A \text { is positive definite (i.e. }\left(A_{k}\right)_{k=1}^{m} \in \Lambda\right)
$$

and in case $A$ is positive definite,

$$
J\left(\left(g_{A_{k}}\left(\cdot-B_{k} b+u_{k}\right)\right)\right)=\left(\frac{\operatorname{det}(A)}{\prod_{k=1}^{m} \operatorname{det}\left(A_{k}\right)^{c_{k}}}\right)^{-\frac{1}{2}} e^{\pi\left(\left\langle A^{-1} v, v\right\rangle-\sum_{k=0}^{m+1} c_{k}\left\langle A_{k} u_{k}, u_{k}\right\rangle\right)} .
$$

In terms of the translation parameters $u_{k}$ (for $k=0, \ldots, m+1$ ), the term inside the exponential is a quadratic form. Hence its infimum is 0 if the quadratic form is positive semi-definite and $-\infty$ else. In the latter case, we get that the infimum of $J$ is zero because of certain non-centered Gaussian functions, while in the former case we get that $J$ takes smaller values on centered Gaussians $\left(g_{A_{k}}\right)$ than on their translates. In short,

$$
\inf _{\mathcal{G}} J \in\left\{0, \inf _{\mathcal{C}} J\right\} .
$$

Proposition 2.7. Suppose that $\mathcal{Q}$ is positive definite on ker $B_{+}$(which guarantees that $J$ is finite for some centered Gaussian functions) and that $\inf _{\mathcal{G}} J>0$. Assuming the notation that is involved in (2.7) and (2.11), the following assertions hold true:

(1) If $\left(A_{k}\right)_{k=1}^{m} \in \Lambda$ then for all $v_{k} \in H_{k}, k=0, \ldots, m+1$, setting $A=\sum_{k=0}^{m+1} c_{k} B_{k}^{*} A_{k} B_{k}$ and $v=\sum_{k=0}^{m+1} c_{k} B_{k}^{*} v_{k}$, it holds

$$
\left\langle A^{-1} v, v\right\rangle \geq \sum_{k=0}^{m+1} c_{k}\left\langle A_{k}^{-1} v_{k}, v_{k}\right\rangle
$$

(2) The map $x \mapsto\left(B_{0} x, B_{1} x, \ldots, B_{m^{+}} x\right)$ from $H$ to $H_{0} \times \cdots \times H_{m^{+}}$is onto.

(3) $\operatorname{dim} H \geq s^{+}(\mathcal{Q})+\sum_{i=1}^{m^{+}} \operatorname{dim} H_{i}$. 
Proof. Since $\inf _{\mathcal{G}} J>0$, reasoning as above on the argument of the exponential term in (2.13) shows that if $v=\sum_{k=1}^{m} c_{k} B_{k}^{*} A_{k} u_{k}$ then

$$
\left\langle A^{-1} v, v\right\rangle \geq \sum_{k=0}^{m+1} c_{k}\left\langle A_{k} u_{k}, u_{k}\right\rangle
$$

Applying this to $u_{k}=A^{-1} v_{k}(k=0,1, \ldots, m+1)$ concludes the proof of the first item.

Let us address the second part of the claim. By duality, our goal is to show that the $\operatorname{map}\left(v_{0}, \ldots, v_{m^{+}}\right) \mapsto \sum_{i=0}^{m^{+}} c_{i} B_{i}^{*} v_{i}$ is injective. So we assume that $\sum_{i=0}^{m^{+}} c_{i} B_{i}^{*} v_{i}=0$, and we want to prove that $v_{0}=\cdots=v_{m^{+}}=0$ (recall that $c_{i} \neq 0$ ). If we set $v_{j}=0$ for $m^{+}<j \leq m+1$, it holds that $0=\sum_{k=0}^{m+1} c_{k} B_{k}^{*} v_{k}$. Thanks to Proposition 2.2, we may find $\left(A_{k}\right)_{k=1}^{m} \in \Lambda$ and apply the first item of the present Proposition 2.7, it gives that

$$
0=\left\langle A^{-1} 0,0\right\rangle \geq \sum_{k=0}^{m+1} c_{k}\left\langle A_{k}^{-1} v_{k}, v_{k}\right\rangle=\sum_{i=0}^{m^{+}} c_{i}\left\langle A_{i}^{-1} v_{i}, v_{i}\right\rangle
$$

Since $c_{i}>0$ for $0 \leq i \leq m^{+}$, we deduce that $\left\langle A_{i}^{-1} v_{i}, v_{i}\right\rangle=0$, thus $v_{i}=0$.

Eventually the third point of the claim is a direct consequence of the second one (surjectivity implies that the dimension of the target space is not bigger than that of the initial space, and $s^{+}(\mathcal{Q})=\operatorname{dim} H_{0}$ by $\left.(2.9)\right)$.

Proposition 2.8. Assume that $\mathcal{Q}$ is positive definite on $\operatorname{ker} B_{+}$and that $\inf _{\mathcal{C G}} J>0$. Then $B_{+}$is surjective.

Proof. We proceed by contradiction. Assume that $B_{+}$is not onto. Then for some $i \in$ $\left\{1,2, \ldots, m^{+}\right\}$and $v \in H_{i} \backslash\{0\}$, the vector

$$
(0, \ldots, 0, \underbrace{v}_{i \text {-th component }}, 0, \ldots, 0) \in H_{1} \times \cdots \times H_{m^{+}}
$$

is not in the image of the $B_{+}=\left(B_{1}, \ldots, B_{m^{+}}\right): H \rightarrow H_{1} \times \cdots \times H_{m^{+}}$. Fix such $i$ and $v$ and let $P_{(\mathbb{R} v)^{\perp}}: H_{i} \rightarrow H_{i} \cap(\mathbb{R} v)^{\perp}$ be an orthogonal projection. Put $\tilde{H}_{i}=H_{i} \cap(\mathbb{R} v)^{\perp}$ and

$$
\tilde{B}_{i}=P_{(\mathbb{R} v)^{\perp}} B_{i}: H \rightarrow \tilde{H}_{i} .
$$

From the surjective maps $B_{1}, \ldots, B_{i-1}, \tilde{B}_{i}, B_{i+1}, \ldots, B_{m^{+}}$, we construct a map $\tilde{B}_{+}=$ $\left(B_{1}, \ldots, B_{i-1}, \tilde{B}_{i}, B_{i+1}, \ldots, B_{m^{+}}\right)$from $H$ to $H_{1} \times \cdots \times H_{i-1} \times \tilde{H}_{i} \times H_{i+1} \times \cdots \times H_{m^{+}}$.

Now we show that $Q$ is positive definite on ker $\tilde{B}_{+}$. To this end, take any $x \in H$ for which $\left(B_{1} x, \ldots B_{i-1} x, \tilde{B}_{i} x, B_{i+1} x, \ldots, B_{m^{+}} x\right)=(0, \ldots, 0)$. Hence

$$
B_{+} x=\left(B_{1} x, \ldots, B_{m^{+}} x\right) \in(0, \ldots, 0, \underbrace{\mathbb{R} v}_{i \text {-th component }}, 0, \ldots, 0) \text {, }
$$

but since $(0, \ldots, 0, v, 0, \ldots, 0)$ is not in the image of $B_{+}=\left(B_{1}, \ldots, B_{m^{+}}\right)$, we must have $B_{+} x=0$. By assumption, $Q$ is positive definite on ker $B_{+}$, which gives $\langle Q x, x\rangle>0$ if $x \neq 0$.

Applying Proposition 2.2 to $Q$ and the maps $B_{1}, \ldots, B_{i-1}, \tilde{B}_{i}, B_{i+1}, \ldots, B_{m}$ we have positive maps $A_{k}: H_{k} \rightarrow H_{k}$ for $k \neq i$ and $\tilde{A}_{i}: \tilde{H}_{i} \rightarrow \tilde{H}_{i}$ such that the map

$$
Q+c_{i} \tilde{B}_{i}^{*} \tilde{A}_{i} \tilde{B}_{i}+\sum_{\substack{1 \leq k \leq m \\ k \neq i}} c_{k} B_{k}^{*} A_{k} B_{k} \quad \text { is positive. }
$$

For $t>0$ define a positive map $A_{i}^{(t)}=P_{(\mathbb{R} v)^{\perp}}^{*} \tilde{A}_{i} P_{(\mathbb{R} v)^{\perp}}+t v v^{*}: H_{i} \rightarrow H_{i}$. Note that

$\lim _{t \rightarrow 0^{+}} \operatorname{det}\left(Q+c_{i} B_{i}^{*} A_{i}^{(t)} B_{i}+\sum_{\substack{1 \leq k \leq m \\ k \neq i}} c_{k} B_{k}^{*} A_{k} B_{k}\right)=\operatorname{det}\left(Q+c_{i} \tilde{B}_{i}^{*} \tilde{A}_{i} \tilde{B}_{i}+\sum_{\substack{1 \leq k \leq m \\ k \neq i}} c_{k} B_{k}^{*} A_{k} B_{k}\right)>0$ 
while $\lim _{t \rightarrow 0+} \operatorname{det} A_{i}^{(t)}=0$. Therefore using the formula (2.2) we see that

$$
\lim _{t \rightarrow 0^{+}} J\left(g_{A_{1}}, \ldots, g_{A_{i-1}}, g_{A_{i}^{(t)}}, g_{A_{i+1}}, \ldots, g_{A_{m}}\right)=0,
$$

where $g_{A}(x)$ is a centered Gaussian function $e^{-\pi\langle A x, x\rangle}$.

2.5. Case analysis and non-degeneracy hypotheses. The goal of this section is to give a full view of the cases when the best constant in inverse Brascamp-Lieb inequalities can be computed with Gaussian functions only.

Case 0.0: The restriction of $\mathcal{Q}$ to $\operatorname{ker} B_{+}$is not positive definite and $B_{+}$is not surjective. In this case, Lemma 2.1(i) implies that $\min J=0$. On the other hand, Proposition 2.2 implies that $\inf _{\mathcal{C G}} J=+\infty$, or equivalently $\Lambda=\emptyset$, which combined with (2.12) implies that also $\inf _{\mathcal{G}} J=+\infty$. Gaussian functions do not allow to compute the infimum of $J$.

Case 0.1: The restriction of $\mathcal{Q}$ to $\operatorname{ker} B_{+}$is not positive definite and $B_{+}$is surjective. Proposition 2.4 ensures that $\inf J=+\infty$. The functional is always infinite. In a very degenerate sense, centered Gaussian functions allow to compute the infimum of $J$.

Case 1.0.0: The restriction of $\mathcal{Q}$ to $\operatorname{ker} B_{+}$is positive definite, $\operatorname{dim} H<s^{+}(\mathcal{Q})+$ $\sum_{i=1}^{m^{+}} \operatorname{dim} H_{i}$ and $B_{+}$is not surjective. By Lemma 2.1(i), min $J=0$. Proposition 2.8 ensures that $\inf _{\mathcal{C G}} J=0$.

Case 1.0.1: $\mathcal{Q}$ is positive definite on $\operatorname{ker} B_{+}, \operatorname{dim} H<s^{+}(\mathcal{Q})+\sum_{i=1}^{m^{+}} \operatorname{dim} H_{i}$ and $B_{+}$is surjective. Proposition 2.7 gives $\inf _{\mathcal{G}} J=0$. However, in this case the value of $\inf _{\mathcal{C} G} J$ is not always 0 . We will give examples later.

Case 1.1: $\mathcal{Q}$ is positive definite on $\operatorname{ker} B_{+}$and $\operatorname{dim} H \geq s^{+}(\mathcal{Q})+\sum_{i=1}^{m^{+}} \operatorname{dim} H_{i}$. This is our last case, and in a sense the only non-degenerate one. Dealing with it is the main part of the work. We postpone the proof of the following statement to the next section, in order to discuss its consequences first.

Theorem 2.9. If $\mathcal{Q}$ is positive definite on $\operatorname{ker} B_{+}$and

$$
\operatorname{dim} H \geq s^{+}(\mathcal{Q})+\sum_{i=1}^{m^{+}} \operatorname{dim} H_{i}
$$

then $\inf J=\inf _{\mathcal{C G}} J$.

So under the above hypothesis, centered Gaussian functions allow to compute the optimal constant in inverse Brascamp-Lieb inequalities. When the hypothesis of the theorem is not verified, $\inf J$ can only be 0 or $+\infty$.

Remark 2.10. Assume (2.7), (2.8) and the notation (2.11). Then

$$
\mathcal{Q}(x)=\pi c_{0}\left\langle A_{0} B_{0} x, B_{0} x\right\rangle+\pi c_{m+1}\left\langle A_{m+1} B_{m+1} x, B_{m+1} x\right\rangle,
$$

which ensures that

$$
\operatorname{ker}\left(B_{0}, \ldots, B_{m^{+}}\right) \subset\{x \in H: \mathcal{Q}(x) \leq 0\} \cap \bigcap_{i=1}^{m^{+}} \operatorname{ker} B_{i}
$$

Hence $\left(B_{0}, \ldots, B_{m^{+}}\right)$is injective when $\mathcal{Q}$ is positive on ker $B_{+}$. Together with (2.9), this implies that $\operatorname{dim} H \leq s^{+}(\mathcal{Q})+\operatorname{dim} H_{1}+\cdots+\operatorname{dim} H_{m^{+}}$. Since the hypotheses of the above theorem provide the converse inequality, they imply that $\operatorname{dim} H=s^{+}(\mathcal{Q})+\operatorname{dim} H_{1}+\cdots+$ $\operatorname{dim} H_{m^{+}}$, and that $\left(B_{0}, \ldots, B_{m^{+}}\right)$is a bijection. 


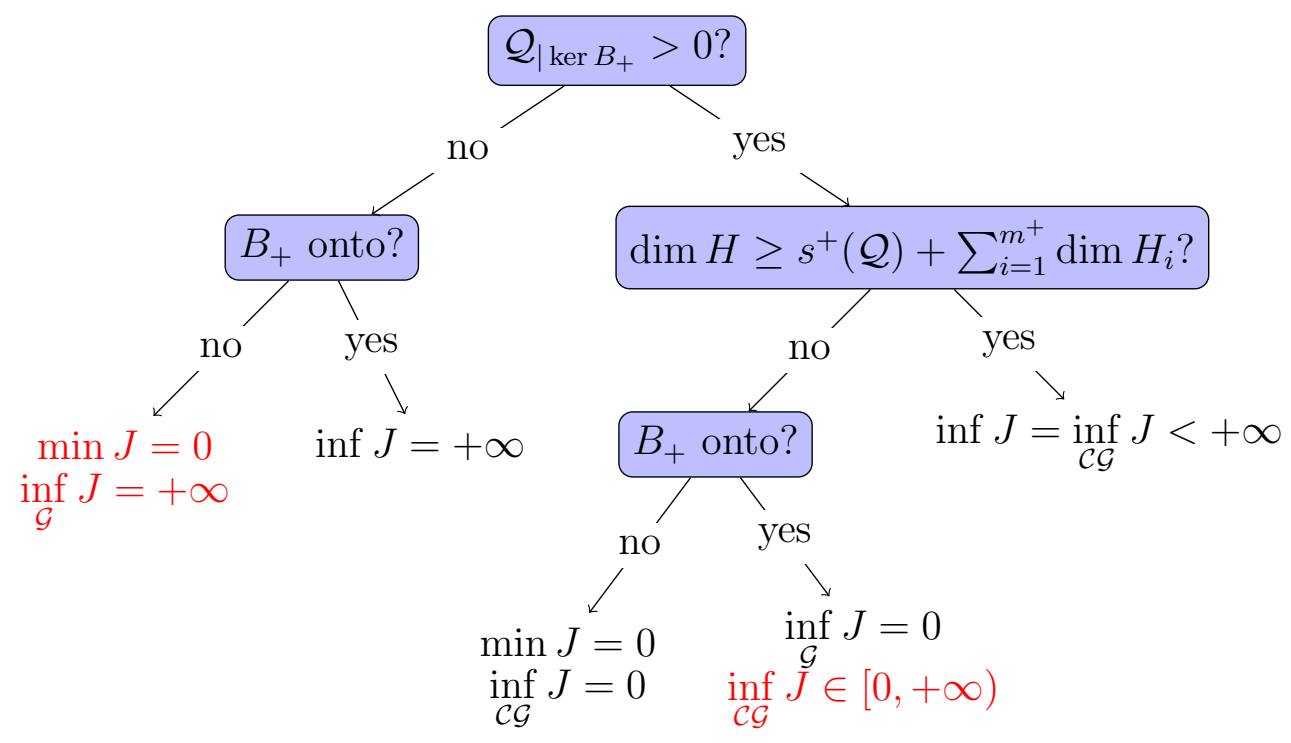

FiguRE 1. Summary of the case analysis

Let us mention variants of the above theorem, which consist in grouping a bit differently the various possible cases. A first variant is Theorem 1.4, as stated in the introduction. Another one is given next. It means that under the assumption that the functional $J$ is finite for some Gaussian functions, the optimal constant can be computed using noncentered Gaussian functions only.

Theorem 2.11. If $\mathcal{Q}$ is positive definite on $\operatorname{ker} B_{+}$then $\inf J=\inf _{\mathcal{G}} J$.

Next we provide examples of the cases when the Gaussian minimizers principle fails.

Example 2.12. Consider the very simple case of the functional

$$
J(f, g):=\frac{\int_{\mathbb{R}^{2}} f(x) g(x) d x d y}{\int_{\mathbb{R}} f \times \int_{\mathbb{R}} g} .
$$

Here $m^{+}=m=2, c_{1}=c_{2}=1, \mathcal{Q}=0$ and $B_{1}(x, y)=B_{2}(x, y)=x$. The map $B_{+}: \mathbb{R}^{2} \rightarrow \mathbb{R}^{2}$ is given by $B_{+}(x, y)=(x, x)$. It is not surjective, and $\mathcal{Q}$ is not positive definite on $\operatorname{ker} B_{+}=\{0\} \times \mathbb{R}$. So we are in the setting of Case 0.0 above.

By Fubini $J(f, g)=+\infty \times \int_{\mathbb{R}} f(x) g(x) d x /\left(\int f \times \int g\right)$ which is equal to 0 if the supports of $f$ and $g$ are disjoint, and is equal to $+\infty$ for Gaussian functions.

Example 2.13 (Reversed hypercontractivity). Borell's reverse Gaussian hypercontractivity [18] states that for any $p, q \in(-\infty, 1)$ the operators of the Ornstein-Uhlenbeck semigroup $P_{t} f(x)=\int_{\mathbb{R}} f\left(e^{-t} x+\sqrt{1-e^{-2 t}} y\right) \gamma(d y)$, where $\gamma$ is a standard Gaussian measure, satisfy

$$
\left\|P_{t} f\right\|_{L^{q}(\gamma)} \geq\|f\|_{L^{p}(\gamma)}
$$

for all positive functions $f \in L^{1}(\gamma)$ if and only if $e^{-2 t} \leq \frac{1-p}{1-q}$. Excluding the case when either $p, q$ or $t$ is 0 and using the fact that for $q \in(-\infty, 1)$ and $h \in L^{q},\|h\|_{L^{q}}=$ $\inf \left\{\int h k: k>0, \int k^{q^{\prime}}=1\right\}$ where $q^{\prime}=q /(q-1)$, the above estimate can be restated as follows: let $n=2, m=2, n_{1}=n_{2}=1, B_{1}\left(x_{1}, x_{2}\right)=x_{1}, B_{2}\left(x_{1}, x_{2}\right)=x_{2}, c_{1}=1 / p \in$ $\mathbb{R} \backslash[0,1], c_{2}=1 / q^{\prime} \in \mathbb{R} \backslash[0,1], t>0$ and

$$
Q=\frac{1}{2 \pi\left(1-e^{-2 t}\right)}\left(\begin{array}{cc}
1-\left(1-e^{-2 t}\right) c_{1} & -e^{-t} \\
-e^{-t} & 1-\left(1-e^{-2 t}\right) c_{2}
\end{array}\right)
$$


Then for the corresponding functional

$$
J(f, g)=\int_{\mathbb{R}^{2}} e^{-\pi\langle Q x, x\rangle} f^{c_{1}}\left(x_{1}\right) g^{c_{2}}\left(x_{2}\right) d x_{1} d x_{2}\left(\int f\right)^{-c_{1}}\left(\int g\right)^{-c_{2}},
$$

we have inf $J=(2 \pi)^{1-\frac{c_{1}+c_{2}}{2}} \sqrt{1-e^{-2 t}}$ if and only if $c_{1} c_{2} \operatorname{det} Q \geq 0$.

Now, let us focus on the a specific example $c_{1}=c_{2}=2$. In this case $B_{+}\left(x_{1}, x_{2}\right)=$ $\left(x_{1}, x_{2}\right)$. Hence $B_{+}$is surjective and $Q$ is positive definite on $\operatorname{ker} B_{+}=\{0\}$. Condition (2.5) is violated if and only if $s^{+}(Q)>0$, which is equivalent to

$$
\operatorname{tr}(Q)>0 \text { or } \operatorname{det}(Q)<0 \text {. }
$$

Actually, in our case $\operatorname{tr}(Q)>0$ implies $\operatorname{det}(Q)<0$. A simple calculation shows that $s^{+}(Q)>0$ holds if and only if $e^{-2 t}>1 / 4$. Thus, whenever $e^{-2 t}>1 / 4$, we are in Case 1.0.1 above, and $\inf J=\inf _{\mathcal{G}} J=0$. Besides, Borell's result asserts that inf $J=$ $(2 \pi)^{-1} \sqrt{1-e^{-2 t}}$ provided $e^{-2 t} \leq 1 / 4$. Next we claim that

$$
\inf _{\mathcal{C} \mathcal{G}} J= \begin{cases}(2 \pi)^{-1} \sqrt{1-e^{-2 t}} & \text { if } e^{-2 t} \in\left(\frac{1}{4}, \frac{1}{2}\right] \\ 0 & \text { if } e^{-2 t} \in\left(\frac{1}{2}, 1\right) .\end{cases}
$$

This is an illustration of Case 1.0.1 above: $\inf J=\inf _{\mathcal{G}} J=0$ but $\inf _{\mathcal{C G}} J$ can be 0 in some cases, and positive in some other cases.

It remains to prove the claim. Put $f(x)=e^{-a x^{2} / 2}$ and $g(x)=e^{-b x^{2} / 2}$ for some $a, b>0$. Then

$$
J(f, g)^{2}= \begin{cases}\frac{(2 \pi)^{2-\left(c_{1}+c_{2}\right)} a^{c_{1}} b^{c_{2}}\left(1-e^{-2 t}\right)^{2}}{\operatorname{det}\left(\begin{array}{cc}
1+\left(1-e^{-2 t}\right) c_{1}(a-1) & -e^{-t} \\
-e^{-t} & 1+\left(1-e^{-2 t}\right) c_{2}(b-1)
\end{array}\right)} & \text { if det }>0, \\
+\infty & \text { otherwise. }\end{cases}
$$

Restricting our attention to the case $c_{1}=c_{2}=2$,

$J(f, g)^{2}= \begin{cases}(2 \pi)^{-2}\left(1-e^{-2 t}\right) \frac{a^{2} b^{2}}{4(1+a b)\left(1-e^{-2 t}\right)-3+2\left(2 e^{-2 t}-1\right)(a+b)} & \text { if the denominator is positive } \\ +\infty & \text { otherwise. }\end{cases}$

In the case $e^{-2 t} \in(1 / 2,1)$ we show that $\inf _{a, b>0} J(f, g)^{2}=0$ by checking that

$$
\begin{array}{r}
\sup _{a, b>0} \frac{4(1+a b)\left(1-e^{-2 t}\right)-3+2\left(2 e^{-2 t}-1\right)(a+b)}{a^{2} b^{2}} \\
\geq \sup _{a>0, b=1 / a} 8\left(1-e^{-2 t}\right)-3+2\left(2 e^{-2 t}-1\right)(a+1 / a)=+\infty .
\end{array}
$$

In the case $e^{-2 t} \in(1 / 4,1 / 2]$ we will have $\inf _{a, b>0} J(f, g)=(2 \pi)^{-1} \sqrt{1-e^{-2 t}}$ if we show

$$
\sup _{a, b>0} \frac{4(1+a b)\left(1-e^{-2 t}\right)-3+2\left(2 e^{-2 t}-1\right)(a+b)}{a^{2} b^{2}}=1 .
$$

Put $\lambda=2-4 e^{-2 t} \in[0,1)$. Since $a+b$ is multiplied by the coefficient $2\left(2 e^{-2 t}-1\right)=-\lambda \leq 0$, we can use the inequality $a+b \geq 2 \sqrt{a b}$ to calculate the supremum in (2.14) as follows:

$$
\sup _{a, b>0} \frac{\lambda-1-\lambda(a+b)+(\lambda+2) a b}{a^{2} b^{2}}=\sup _{x=(a b)^{-1 / 2}>0}(\lambda-1) x^{4}-2 \lambda x^{3}+(\lambda+2) x^{2}=: \sup _{x>0} \varphi(x) \text {. }
$$

Since

$$
\varphi^{\prime}(x)=-4(1-\lambda) x(x-1)\left(x-\frac{\lambda+2}{2(\lambda-1)}\right),
$$

$\varphi$ is increasing on $(0,1]$ and decreasing on $[1, \infty)$ and hence $\sup _{x>0} \varphi(x)=\varphi(1)=1$. 
We conclude this section with the analysis of degenerate and non-degenerate cases for the inverse convolution inequality.

Example 2.14 (Reverse Young inequality). As mentioned in the introduction: for $p, q, r \in$ $(0,1]$ such that $1+1 / r=1 / p+1 / q$, and positive functions on $\mathbb{R}^{n}$, Brascamp and Lieb have proved that

$$
\|f * g\|_{r} \geq\left(\frac{C_{p} C_{q}}{C_{r}}\right)^{n}\|f\|_{p}\|g\|_{q}
$$

holds where $C_{t}=|t|^{1 / t} /\left|t^{\prime}\right|^{1 / t^{\prime}}$, and the constant is optimal. Our goal here is to discuss extensions to negative exponents.

Using a duality type argument, a change of functions and the fact that $C_{p^{\prime}}=1 / C_{p}$ we can reformulate the above result as follows: if $p, q \in(0,1]$ and $r^{\prime} \in(-\infty, 0)$ verify $1 / p+1 / q+1 / r^{\prime}=2$ then for all positive integrable functions $f, g, h$,

$$
\int_{\left(\mathbb{R}^{n}\right)^{2}} f(x-y)^{\frac{1}{p}} g(y)^{\frac{1}{q}} h(x)^{\frac{1}{r^{\prime}}} d x d y \geq\left(C_{p} C_{q} C_{r^{\prime}}\right)^{n}\left(\int f\right)^{\frac{1}{p}}\left(\int g\right)^{\frac{1}{g}}\left(\int h\right)^{\frac{1}{r^{\prime}}} .
$$

Simple changes of variables as $\int F(x-y) G(y) H(x) d x d y=\int F(z) G(x-z) H(x) d x d z$ show that $p, q$ and $r^{\prime}$ play symmetric roles. Therefore the convolution inequality is also true when $1 / p+1 / q+1 / r^{\prime}=2$ and among the three numbers $p, q, r^{\prime}$, two are in $(0,1]$ and one is in $(-\infty, 0)$, (which is more general than $p, q, r \in(0,1])$. However no non-trivial inequality holds beyond this range of indices, as we show next.

The condition $1 / p+1 / q+1 / r^{\prime}=2$ is necessary (applying the inequality to $f(\lambda \cdot), g(\lambda \cdot)$, $h(\lambda \cdot)$ for $\lambda>0$ and changing variables $(x, y)=\lambda^{-1}(X, Y)$ gives it). Consider the three surjective maps from $\mathbb{R}^{2 n}$ to $\mathbb{R}^{n}$ defined by $B_{1}(x, y)=x-y, B_{2}(x, y)=y, B_{3}(x, y)=x$, and the numbers $c_{1}=1 / p, c_{2}=1 / q$ and $c_{3}=1 / r^{\prime}$. The above analysis of degenerate cases shows that one should focus on the map $B_{+}=\left(B_{i}\right)_{i: c_{i}>0}$. If $p, q, r^{\prime}$ are positive then $B_{+}$ is not surjective and the only possible constant in the convolution inequality is 0 . If only one among the three number $p, q, r^{\prime}$ is positive, then $B_{+}$is surjective but not injective and we are in Case 0.1, meaning that the functional under study never takes finite values.

\section{Proof of Theorem 2.9}

3.1. Decomposition of the kernel $\exp (-\mathcal{Q})$. The positive and negative parts of a quadratic form $\mathcal{Q}$ play different roles, as do the functions $f_{i}$ with $i \leq m^{+}$and the functions $f_{j}$ with $j>m^{+}$. Although there is no canonical decomposition of $H$ into subspaces on which $\mathcal{Q}$ is, respectively, positive and negative definite, Condition (2.4) provides a natural candidate for a subspace on which $\mathcal{Q}$ is positive definite. This leads to the following result:

Lemma 3.1. The following two assertions are equivalent:

(1) (i) $\mathcal{Q}$ is positive definite on $\operatorname{ker} B_{+}$and (ii) $\operatorname{dim} H \geq s^{+}(\mathcal{Q})+\sum_{i=1}^{m^{+}} \operatorname{dim} H_{i}$.

(2) There exist vector spaces $H_{0}, H_{m+1}$, surjective linear maps $B_{0}: H \rightarrow H_{0}$ and $B_{m+1}: H \rightarrow H_{m+1}$, and positive definite quadratic forms $\mathcal{Q}_{+}$on $H_{0}$ and $\mathcal{Q}_{-}$on $H_{m+1}$ such that:

- $\left(B_{0}, B_{+}\right): H \rightarrow H_{0} \times \cdots \times H_{m^{+}}$is bijective,

- ker $B_{+} \subset \operatorname{ker} B_{m+1}$,

- for all $x \in H, \quad \mathcal{Q}(x)=\mathcal{Q}_{+}\left(B_{0} x\right)-\mathcal{Q}_{-}\left(B_{m+1} x\right)$.

Remark 3.2. The above decomposition of $\mathcal{Q}$ is more specific than the ones introduced in Subsection 2.4 in (2.7). Although we have used for convenience the same notation $B_{0}$ and $B_{m+1}$ there, they do not necessarily represent the same maps as in the above 
lemma. However no confusion will be possible, since from now on we will only use the decomposition of Lemma 3.1.

Proof. We start with $(2) \Longrightarrow(1)$ : For any $x \in \operatorname{ker} B_{+} \subset$ ker $B_{m+1}$, it holds

$$
\mathcal{Q}(x)=\mathcal{Q}_{+}\left(B_{0} x\right)-\mathcal{Q}_{-}\left(B_{m+1} x\right)=\mathcal{Q}_{+}\left(B_{0} x\right) \geq 0 .
$$

Moreover, if $\mathcal{Q}(x)=0$, using that $\mathcal{Q}_{+}$is definite positive, we deduce that $B_{0} x=0$. It follows that $x$ belongs to $\operatorname{ker} B_{0} \cap \operatorname{ker} B_{+}$, which is equal to $\{0\}$ by hypothesis. Thus we have shown that $\mathcal{Q}$ is positive definite on $\operatorname{ker} B_{+}$.

It remains to prove (1)(ii). By hypothesis, $\left(B_{0}, B_{+}\right)$is a linear isomorphism, which implies that

$$
\operatorname{dim} H=\sum_{i=0}^{m^{+}} \operatorname{dim} H_{i} .
$$

Therefore, it is enough to show that $s^{+}(\mathcal{Q}) \leq \operatorname{dim} H_{0}$. Since $\mathcal{Q}_{-}$is positive definite, $\mathcal{Q}$ is negative semi-definite on $\operatorname{ker} B_{0}$, and hence

$$
s^{+}(\mathcal{Q}) \leq \operatorname{dim} H-\operatorname{dim} \operatorname{ker} B_{0}=\operatorname{dim} H_{0} .
$$

Now we prove that $(1) \Longrightarrow(2)$. Set

$$
H_{0}=\operatorname{ker} B_{+}=\bigcap_{i=1}^{m^{+}} \operatorname{ker} B_{i}
$$

Note that (1) implies $\operatorname{dim} H_{0}=s^{+}(\mathcal{Q})$. Indeed,

$$
\operatorname{dim} H_{0}=\operatorname{dim} \operatorname{ker} B_{+}=\operatorname{dim} H-\operatorname{dim} \operatorname{Im} B_{+} \geq \operatorname{dim} H-\sum_{i=1}^{m^{+}} \operatorname{dim} H_{i} \geq s^{+}(\mathcal{Q}),
$$

where the last inequality follows from (1)(ii). The converse inequality follows from Sylvester's theorem since $\mathcal{Q}$ is positive definite on $H_{0}$.

Now consider the subspace

$$
H_{0}^{\perp_{\mathcal{Q}}}=\left\{x \in H: \forall y \in H_{0}, \mathcal{Q}(x, y)=0\right\},
$$

where we also denote by $\mathcal{Q}(\cdot, \cdot)$ the symmetric bilinear form associated with the quadratic form $\mathcal{Q}$. Since $\mathcal{Q}$ is positive definite on $H_{0}$, we have

$$
H_{0} \cap H_{0}^{\perp \mathcal{Q}} \subseteq\left\{x \in H_{0}: \mathcal{Q}(x, x)=0\right\}=\{0\} .
$$

As a general fact, $\operatorname{dim} H_{0}^{\perp_{\mathcal{Q}}} \geq \operatorname{dim} H-\operatorname{dim} H_{0}$, therefore

$$
H_{0} \oplus H_{0}^{\perp_{\mathcal{Q}}}=H \text {. }
$$

Consider the projection $P: H \rightarrow H$ onto $H_{0}$ with kernel $H_{0}^{\perp}{ }^{\mathcal{Q}}$. Then Id $-P: H \rightarrow H$ is the projection onto $H_{0}^{\perp \mathcal{Q}}$ with $\operatorname{ker}(\mathrm{Id}-P)=H_{0}$ and

$$
\mathcal{Q}(x)=\mathcal{Q}(P x)+\mathcal{Q}((\operatorname{Id}-P) x) .
$$

Next, note that $\mathcal{Q}$ is negative semi-definite on $H_{0}^{\perp \mathcal{Q}}$. Indeed, suppose that for some $0 \neq x \in H_{0}^{\perp_{\mathcal{Q}}}, \mathcal{Q}(x)>0$. Then for all $\lambda \in \mathbb{R}$ and $y \in H_{0}$,

$$
\mathcal{Q}(\lambda x+y)=\lambda^{2} \mathcal{Q}(x)+2 \lambda \mathcal{Q}(x, y)+\mathcal{Q}(y)=\lambda^{2} \mathcal{Q}(x)+\mathcal{Q}(y)>0
$$

whenever $\lambda \neq 0$ or $y \neq 0$, which thanks to (3.3) is equivalent to $\lambda x+y \neq 0$. In this way $\mathcal{Q}$ would be positive definite on the subspace $H_{0} \oplus \operatorname{span}\{x\}$ which has dimension strictly larger than $s^{+}(\mathcal{Q})$ and thus it contradicts Sylvester's theorem. 
Further on, as a general fact, the radical of $\mathcal{Q}$

$$
\operatorname{rad} \mathcal{Q}=\{x \in H: \forall y \in H, \mathcal{Q}(x, y)=0\}
$$

is a subspace in $H_{0}^{\perp_{\mathcal{Q}}}$. Consider

$$
H_{m+1}=H_{0}^{\perp \mathcal{Q}} / \mathrm{rad} \mathcal{Q}
$$

and the maps $B_{0}: H \rightarrow H_{0}$ and $B_{m+1}: H \rightarrow H_{m+1}$ defined for $x \in H$ by

$$
\begin{aligned}
& B_{0}(x)=P(x), \\
& B_{m+1}(x)=\pi_{H_{0}^{\perp \mathcal{Q}} \rightarrow H_{0}^{\perp \mathcal{Q}} / \operatorname{rad} \mathcal{Q}}((\operatorname{Id}-P)(x)),
\end{aligned}
$$

where $\pi_{H_{0}^{\perp \mathcal{Q}} \rightarrow H_{0}^{\perp \mathcal{Q}} / \mathrm{rad} \mathcal{Q}}$ is the natural quotient map from $H_{0}^{\perp \mathcal{Q}}$ to $H_{0}^{\perp \mathcal{Q}} / \mathrm{rad} \mathcal{Q}$. Finally, consider the following positive definite quadratic forms

$$
\begin{aligned}
& \mathcal{Q}_{+}=\left.\mathcal{Q}\right|_{H_{0}}: H_{0} \rightarrow \mathbb{R}, \\
& \mathcal{Q}_{-}: H_{m+1} \rightarrow \mathbb{R}, \quad \mathcal{Q}_{-}(x+\operatorname{rad} \mathcal{Q})=-\mathcal{Q}(x) \text { for } x \in H_{0}^{\perp_{\mathcal{Q}}} .
\end{aligned}
$$

Then the decomposition (3.4) becomes

$$
\mathcal{Q}(x)=\mathcal{Q}_{+}\left(B_{0} x\right)-\mathcal{Q}_{-}\left(B_{m+1} x\right) .
$$

Next, let us establish the claimed properties of the linear maps which appear in the above decomposition of $\mathcal{Q}$.

The non-degeneracy conditions (1)(i) and (1)(ii) imply that the map

$$
B_{0+}:=\left(B_{0}, B_{1}, \ldots, B_{m^{+}}\right): H \rightarrow H_{0} \times H_{1} \times \cdots \times H_{m^{+}} \text {is a linear isomorphism. }
$$

Indeed ker $B_{0} \cap$ ker $B_{+}=H_{0}^{\perp Q} \cap H_{0}=\{0\}$, hence $B_{0+}$ is injective. The dimension condition (1)(ii), once rewritten as $\operatorname{dim} H \geq \sum_{i=0}^{m^{+}} \operatorname{dim} H_{i}$, shows that $B_{0+}$ is an isomorphism.

Note also that ker $B_{m+1}=H_{0}+\operatorname{rad} \mathcal{Q}$ and therefore

$$
\text { ker } B_{+}=H_{0} \subseteq \operatorname{ker} B_{m+1} .
$$

This concludes the proof of the claimed properties.

The simple lemma stated below establishes the following consequence of (3.2):

$$
\text { if } F \subseteq H_{1} \times \cdots \times H_{m^{+}} \text {is compact, then } B_{m+1}\left(B_{+}^{-1}(F)\right) \text { is compact. }
$$

Lemma 3.3. Let $S: X \rightarrow Y$ and $T: X \rightarrow Z$ be linear maps between finite dimensional linear spaces $X, Y, Z$ and $F \subseteq Y$ be a compact set. If $\operatorname{ker} S \subseteq \operatorname{ker} T$ then $T\left(S^{-1}(F)\right)$ is a compact subset of $Z$.

Proof. Put $V=\operatorname{ker} S$ and let $\pi: X \rightarrow X / V$ be the natural quotient map. Define $\tilde{S}: X / V \rightarrow$ $Y$ and $\tilde{T}: X / V \rightarrow Z$ as

$$
\tilde{S}(x+V)=S x, \quad \tilde{T}(x+V)=T x
$$

(these definitions are correct since the kernels of $S$ and $T$ contain $V$ ), i.e. $\tilde{S} \circ \pi=S$ and $\tilde{T} \circ \pi=T$. Note that ker $\tilde{S}$ is trivial, hence $\tilde{S}$ is a linear an isomorphism onto its range, and thus $G=\tilde{S}^{-1}(F)$ is a compact subset of $X / V$. Finally write

$$
T\left(S^{-1}(F)\right)=\tilde{T}\left(\pi\left(\pi^{-1}\left(\tilde{S}^{-1}(F)\right)\right)\right)=\tilde{T}\left(\tilde{S}^{-1}(F)\right)=\tilde{T}(G)
$$

and use that $\tilde{T}(G)$ is also compact. 
3.2. More on quadratic forms. We start with recalling a simple inequality, which appears in the transportation proof of the Brascamp-Lieb inequalities.

Lemma 3.4. Let $I$ be a finite set, and for each $i \in I$ let $d_{i}>0, L_{i}: H \rightarrow H_{i}$ be linear and onto and $K_{i}: H_{i} \rightarrow H_{i}$ be a linear symmetric definite positive map. Assume that $K:=\sum_{i} d_{i} L_{i}^{*} K_{i} L_{i}>0$. Let $w \in H$, then for all $y_{i} \in H_{i}$ verifying $w=\sum_{i} d_{i} L_{i}^{*} y_{i}$, the following holds

$$
\left\langle K^{-1} w, w\right\rangle \leq \sum_{i} d_{i}\left\langle K_{i}^{-1} y_{i}, y_{i}\right\rangle
$$

There is equality if one chooses $y_{i}:=K_{i} L_{i} K^{-1} w$.

Proof. This is a direct application of the Cauchy-Schwarz inequality:

$$
\begin{aligned}
\left\langle K^{-1} w, w\right\rangle & =\sum_{i} d_{i}\left\langle K^{-1} w, L_{i}^{*} y_{i}\right\rangle=\sum_{i} d_{i}\left\langle K_{i}^{1 / 2} L_{i} K^{-1} w, K_{i}^{-1 / 2} y_{i}\right\rangle \\
& \leq\left(\sum_{i} d_{i}\left\langle K_{i}^{1 / 2} L_{i} K^{-1} w, K_{i}^{1 / 2} L_{i} K^{-1} w\right)^{\frac{1}{2}}\left(\sum_{i} d_{i}\left\langle K_{i}^{-1 / 2} y_{i}, K_{i}^{-1 / 2} y_{i}\right\rangle\right)^{\frac{1}{2}}\right. \\
& =\left(\left\langle\left(\sum_{i} d_{i} L_{i}^{*} K_{i} L_{i}\right) K^{-1} w, K^{-1} w\right\rangle\right)^{\frac{1}{2}}\left(\sum_{i} d_{i}\left\langle K_{i}^{-1} y_{i}, y_{i}\right\rangle\right)^{\frac{1}{2}} \\
& =\left\langle K^{-1} w, w\right\rangle^{\frac{1}{2}}\left(\sum_{i} d_{i}\left\langle K_{i}^{-1} y_{i}, y_{i}\right\rangle\right)^{\frac{1}{2}}
\end{aligned}
$$

When proving Proposition 2.7, we have shown that the quadratic inequality stated as its first conclusion implies that the map $\left(B_{0}, \ldots, B_{m^{+}}\right)$is onto. Our next task is to prove a converse statement, for further use.

Lemma 3.5. Let $c_{1}, \ldots, c_{m^{+}}>0>c_{m^{+}+1}, \ldots, c_{m}$. For $k=1, \ldots, m$, let $B_{k}: H \rightarrow H_{k}$ be a linear surjective map, and let $A_{k}: H_{k} \rightarrow H_{k}$ be symmetric definite positive operator. Assume that $\left(B_{1}, \ldots, B_{m+}\right): H \rightarrow H_{1} \times \cdots \times H_{m^{+}}$is onto.

If $A:=\sum_{k=1}^{m} c_{k} B_{k}^{*} A_{k} B_{k}>0$ and $y=\sum_{k=1}^{m} c_{k} B_{k}^{*} y_{k}$ for some $y_{k} \in H_{k}$, then

$$
\left\langle A^{-1} y, y\right\rangle \geq \sum_{k=1}^{m} c_{k}\left\langle A_{k}^{-1} y_{k}, y_{k}\right\rangle .
$$

There is equality if one chooses $y_{k}:=A_{k} B_{k} A^{-1} y$.

Proof. The statement is derived from the former lemma, after rearranging the terms. By the surjectivity hypothesis, there exits $z \in H$ such that for all $i \leq m^{+}, y_{i}=A_{i} B_{i} z$.

The relationship $y=\sum_{k=1}^{m} c_{k} B_{k}^{*} y_{k}$ can be rewritten as

$$
y+\sum_{m^{+}<j \leq m}\left|c_{j}\right| B_{j}^{*} y_{j}=\left(\sum_{i \leq m^{+}} c_{i} B_{i}^{*} A_{i} B_{i}\right) z .
$$

If we set $K:=\sum_{i \leq m^{+}} c_{i} B_{i}^{*} A_{i} B_{i}, w:=K z, H_{m+1}:=H, B_{m+1}:=\operatorname{Id}_{H}, A_{m+1}:=A$, $y_{m+1}:=y$ and $c_{m+1}=1$, we obtain that

$$
w=\sum_{m^{+}<j \leq m+1}\left|c_{j}\right| B_{j}^{*} y_{j}
$$

With this notation, we may also rewrite the relationship $A=\sum_{k=1}^{m} c_{k} B_{k}^{*} A_{k} B_{k}$ as

$$
K=\sum_{i \leq m^{+}} c_{i} B_{i}^{*} A_{i} B_{i}=A+\sum_{m^{+}<j \leq m}\left|c_{j}\right| B_{j}^{*} A_{j} B_{j}=\sum_{m^{+}<j \leq m+1}\left|c_{j}\right| B_{j}^{*} A_{j} B_{j} .
$$


Observe that $K>0$ holds, as a consequence of $A>0$. Therefore (3.6) and (3.7) allow to apply Lemma 3.4 and to get

$$
\sum_{m^{+}<j \leq m+1}\left|c_{j}\right|\left\langle A_{j}^{-1} y_{j}, y_{j}\right\rangle \geq\left\langle K^{-1} w, w\right\rangle .
$$

By our definitions for $w, K$ and $z$,

$$
\left\langle K^{-1} w, w\right\rangle=\langle K z, z\rangle=\left\langle\sum_{i \leq m^{+}} c_{i} B_{i}^{*} A_{i} B_{i} z, z\right\rangle=\sum_{i \leq m^{+}} c_{i}\left\langle A_{i} B_{i} z, B_{i} z\right\rangle=\sum_{i \leq m^{+}} c_{i}\left\langle y_{i}, A_{i}^{-1} y_{i}\right\rangle .
$$

Since $\left|c_{m+1}\right|\left\langle A_{m+1}^{-1} y_{m+1}, y_{m+1}\right\rangle=\left\langle A^{-1} y, y\right\rangle$ and $c_{j}<0$ for $m^{+}<j \leq m$, the statement of (3.8) gives the claimed inequality. The case of equality is easily verified.

3.3. Preliminaries and general strategy of the proof of Theorem 2.9. The very first step in the proof of Theorem 2.9 is to consider a decomposition of the Gaussian kernel $\exp (-\mathcal{Q})$ as explained before. Namely, thanks to the hypothesis of Theorem 2.9 the assertion (2) from Lemma 3.1 holds true. Therefore we will consider the quadratic forms $\mathcal{Q}_{+}$and $\mathcal{Q}_{-}$together with the maps $B_{0}: H \rightarrow H_{0}$ and $B_{m+1}: H \rightarrow H_{m+1}$ whose existence is ensured by that assertion. Further consider self-adjoint maps $Q_{+}: H_{0} \rightarrow H_{0}$ and $Q_{-}: H_{m+1} \rightarrow H_{m+1}$ which represent the respective quadratic forms, i.e.

$$
\begin{array}{ll}
\mathcal{Q}_{+}(x)=\pi\left\langle Q_{+} x, x\right\rangle & \text { for } x \in H_{0}, \\
\mathcal{Q}_{-}(x)=\pi\left\langle Q_{-} x, x\right\rangle & \text { for } x \in H_{m+1}
\end{array}
$$

For $k=1,2, \ldots, m$ fix measurable functions $f_{k}: H_{k} \rightarrow[0, \infty]$ of integral one. We will deal with the Gaussian kernel $\exp (-\mathcal{Q})$ as two additional functions, namely

$$
\exp (-\mathcal{Q}(x))=\sqrt{\frac{\operatorname{det} Q_{-}}{\operatorname{det} Q_{+}}} f_{0}^{c_{0}}\left(B_{0} x\right) f_{m+1}^{c_{m+1}}\left(B_{m+1} x\right),
$$

where $f_{0}: H_{0} \rightarrow[0, \infty]$ and $f_{m+1}: H_{m+1} \rightarrow[0, \infty]$ are defined as

$$
\begin{aligned}
f_{0}(x) & =\sqrt{\operatorname{det} Q_{+}} \exp \left(-\pi\left\langle Q_{+} x, x\right\rangle\right), \\
f_{m+1}(x) & =\sqrt{\operatorname{det} Q_{-}} \exp \left(-\pi\left\langle Q_{-} x, x\right\rangle\right)
\end{aligned}
$$

and $c_{0}=1, c_{m+1}=-1$. With this notation we have

$$
J\left(f_{1}, \ldots, f_{m}\right)=\sqrt{\frac{\operatorname{det} Q_{-}}{\operatorname{det} Q_{+}}} \int_{H} \prod_{k=0}^{m+1} f_{k}^{c_{k}}\left(B_{k} x\right) d x .
$$

The general strategy is similar to the one of the proof of the direct and reverse BrascampLieb inequality from [11]. Namely we will consider a tuple of centered Gaussian functions $g_{k}$ on $H_{k}(k=0,1, \ldots, m+1)$ of integral one and optimal transport maps $H_{k} \ni x \mapsto y=$ $T_{k}(x) \in H_{k}$ which push forward the density $f_{k}(x) d x$ onto the density $g_{k}(y) d y$. Starting from the maps $T_{k}$, we will build a change of variable map $\theta: H \rightarrow H$ which will allow us to pass from $J\left(f_{1}, \ldots, f_{k}\right)$ in the form of (3.9) to the integral over $H$ involving a Gaussian function only. However, since we aim to bound (3.9) from below, it is crucial that the map $\theta$ is surjective. This point is a substantial technical difficulty which is not present in the transportation proof of the Brascamp-Lieb inequality with positive exponents.

In order to make the above strategy work, we need to restrict $f_{k}$ to carefully chosen classes of functions. There are two reasons behind this: first, we need to ensure existence of optimal transport maps; moreover, it will be convenient to have some regularity of these maps and to have the Monge-Ampère equation satisfied in the classical sense. The second reason is that we need to ensure surjectivity of the map $\theta$. This can be done 
by appropriate choice of supports of the test functions. Finally, the inequality for any integrable functions $f_{k}$ will be obtained via an approximation argument.

We close this subsection with notation and facts concerning convex functions on Euclidean spaces. A standard reference is [39]. In the sequel we write $\operatorname{int} A, \operatorname{cl} A, \operatorname{bd} A$ for the interior, closure and boundary of $A$.

Let $\varphi: \mathbb{R}^{n} \rightarrow \mathbb{R} \cup\{+\infty\}$ be a convex function. The domain of $\varphi$ is

$$
\operatorname{dom} \varphi=\left\{x \in \mathbb{R}^{n}: \varphi(x)<+\infty\right\} .
$$

We say that $\varphi$ is proper if $\operatorname{dom} \varphi \neq \emptyset$. We say that $\varphi$ is closed if the epigraph of $\varphi$, i.e. the set $\left\{(x, y) \in \mathbb{R}^{n+1}: x \in \operatorname{dom} \varphi, y \geq \varphi(x)\right\}$, is a closed subset of $\mathbb{R}^{n}$. A convex function $\varphi$ is closed if and only if it is lower semi-continuous (or, equivalently, for every $\alpha \in \mathbb{R}$, $\left\{x \in \mathbb{R}^{n}: \varphi(x) \leq \alpha\right\}$ is a closed subset of $\mathbb{R}^{n}$.

For $x \in \mathbb{R}^{n}$, the subdifferential $\partial \varphi(x)$ is the set of all vectors $x^{*} \in \mathbb{R}^{n}$, called subgradients, which satisfy

$$
f(y) \geq f(x)+\left\langle x^{*}, y-x\right\rangle \text { for all } y \in H
$$

For $A \subseteq \mathbb{R}^{n}$

$$
\partial \varphi(A)=\bigcup_{x \in A} \partial \varphi(x)
$$

Note that $\partial \varphi(x) \neq \emptyset$ for all $x \in \operatorname{int} \operatorname{dom} \varphi$ (actually for all $x$ in the relative interior of $\operatorname{dom} \varphi)$ and that if $\varphi$ is proper then $\partial \varphi(x)=\emptyset$ for all $x \notin \operatorname{dom} \varphi$. If $\varphi$ is differentiable at $x \in \mathbb{R}^{n}$ then $\partial \varphi(x)$ contains exactly one vector $\nabla \varphi(x)$. The converse statement is also true: for a convex function having a unique subgradient at a given point implies differentiability at that point (see [39, Theorem 25.1]).

If $\varphi$ is proper, we define the Legendre conjugate of $\varphi$ as

$$
\varphi^{*}(y)=\sup _{x \in \operatorname{dom} \varphi}\langle x, y\rangle-\varphi(x),
$$

which is a proper closed convex function on $\mathbb{R}^{n}$. If $\varphi$ is proper and closed then $\left(\varphi^{*}\right)^{*}$ coincides with $\varphi$.

If $\varphi$ is proper and closed then the multi-valued maps $\partial \varphi$ and $\partial \varphi^{*}$ are inverses of each other, i.e.

$$
y \in \partial \varphi(x) \quad \Longleftrightarrow \quad x \in \partial \varphi^{*}(y)
$$

(see [39, Corollary 23.5.1]). In particular, $y \in \partial \varphi\left(\mathbb{R}^{n}\right)$ if and only if $\partial \varphi^{*}(y) \neq \emptyset$ which readily implies that if $\varphi$ is proper and closed then

$$
\operatorname{int} \operatorname{dom} \varphi^{*} \subseteq \partial \varphi\left(\mathbb{R}^{n}\right) \subseteq \operatorname{dom} \varphi^{*}
$$

3.4. Optimal transport map. Here we present a result (formulated as Corollary 3.12) on existence of smooth solutions to the Monge-Ampère equation related to certain class of optimal transport problems. Although the result is most probably well-known to specialists in the theory of the Monge-Ampère equation, we were not able to find a reference where it is explicitly stated. For this reason we explain below how the result can be derived from well-established results in optimal transport and regularity theory of the Monge-Ampère equation.

Let us begin our discussion with the following result of McCann [35], which is a refinement of an earlier result of Brenier [21] (see also references in [35] for related developments):

Theorem 3.6 (Brenier, McCann). Let $\mu$ and $\nu$ be Borel probability measures on $\mathbb{R}^{n}$. 
(i) There exist a Borel probability measure $\gamma$ on $\mathbb{R}^{n} \times \mathbb{R}^{n}$ whose marginals are $\mu$ and $\nu$ (namely, for any Borel set $A \in \mathbb{R}^{n}, \mu(A)=\gamma\left(A \times \mathbb{R}^{n}\right)$ and $\nu(A)=\gamma\left(\mathbb{R}^{n} \times A\right)$ ) and a closed convex function $\varphi: \mathbb{R}^{n} \rightarrow \mathbb{R} \cup\{+\infty\}$ such that the measure $\gamma$ is supported on the graph of the subdifferential of $\varphi$, i.e. the set

$$
\left\{(x, y) \in \mathbb{R}^{n} \times \mathbb{R}^{n}: y \in \partial \varphi(x)\right\} .
$$

(ii) In addition to (i), if $\mu$ vanishes on Borel subsets of $\mathbb{R}^{n}$ Hausdorff dimension $n-1$, then $\varphi$ is differentiable $\mu$-a.e. and the map $\nabla \varphi$ (defined in points where $\varphi$ is differentiable) pushes $\mu$ forward to $\nu$, i.e. for every Borel subset $B \subseteq \mathbb{R}^{n}$,

$$
\nu(B)=\mu\left((\nabla \varphi)^{-1}(B)\right)
$$

(and in fact, the map (id, $\nabla \varphi$ ): $\mathbb{R}^{n} \rightarrow \mathbb{R}^{n} \times \mathbb{R}^{n}$ pushes $\mu$ forward to $\gamma$ ). Moreover, the map $\nabla \varphi$ satisfying (3.12) is uniquely determined $\mu$-a.e. among gradients of convex functions on $\mathbb{R}^{n}$.

The map $\nabla \varphi$ from Theorem 3.6 (ii) is called the Brenier map.

Remark 3.7. $\quad$ (i) For a closed convex function the graph of its subdifferential is a closed subset of $\mathbb{R}^{n} \times \mathbb{R}^{n}$.

(ii) From Theorem 3.6(i) it follows that

$$
\operatorname{supp}(\mu) \subseteq \operatorname{cl}\left\{x \in \mathbb{R}^{n}: \partial \varphi(x) \neq \emptyset\right\}=\operatorname{cldom} \varphi
$$

and

$$
\operatorname{supp}(\nu) \subseteq \operatorname{cl} \partial \varphi\left(\mathbb{R}^{n}\right) .
$$

(iii) The assumption on $\mu$ in Theorem 3.6(ii) is satisfied if $\mu$ is absolutely continuous with respect to the Lebesgue measure on $\mathbb{R}^{n}$.

(iv) Using (3.12) and continuity of the (sub)gradient of a convex function (see e.g. [39, Corollary 24.5.1]) one can show that

$$
\operatorname{supp}(\nu)=\operatorname{cl} \nabla \varphi(\operatorname{supp}(\mu))
$$

(see e.g. the proof of Theorem 2.12 in [42] for details).

(v) By the above item (ii), the exterior of the domain of $\varphi$ has measure $\mu$ zero, and the boundary of $\operatorname{dom} \varphi$ (as the boundary of a convex set in $\mathbb{R}^{n}$ ) has Hausdorff dimension at most $n-1$. Therefore, $\mu$-a.e. differentiability of $\varphi$ follows from the result of Anderson and Klee [2] which says that a convex function on $\mathbb{R}^{n}$ is differentiable everywhere in its domain except for a set of Hausdorff dimension at most $n-1$.

From now on we assume that $\mu$ is a probability measure on $\mathbb{R}^{n}$ with a density $f>0$, and $\nu$ is a probability measure on $\mathbb{R}^{n}$ with a density $g$ which is positive in an open bounded convex set $\Omega$ and $g \equiv 0$ in $\Omega^{c}$. Thanks to Theorem 3.6 we consider a closed convex function $\varphi$ for which $\nabla \varphi$ is the Brenier map which pushes $\mu$ forward to $\nu$. Let us discuss some basic properties of $\varphi$ in this context:

(i) Since $\operatorname{supp}(\mu)=\mathbb{R}^{n}$, it follows from (3.13) that $\operatorname{dom} \varphi=\mathbb{R}^{n}$.

(ii) By the hypothesis that $f>0$, the Lebesgue measure on $\mathbb{R}^{n}$ is absolutely continuous with respect to $\mu$ and hence $\varphi$ is differentiable a.e. in $\mathbb{R}^{n}$.

(iii) By (3.15) it is clear that

$$
\nabla \varphi(x) \in \operatorname{cl} \Omega \text { for all } x \in \mathbb{R}^{n} \text { for which } \nabla \varphi(x) \text { is defined. }
$$

Moreover, by (3.12),$\mu\left((\nabla \varphi)^{-1}(\operatorname{bd} \Omega)\right)=\nu(\operatorname{bd} \Omega)=0$, hence the set $(\nabla \varphi)^{-1}(\operatorname{bd} \Omega)$ has zero Lebesgue measure. Therefore

$$
x \text {-a.e. the following holds: } \varphi \text { is differentiable at } x \text { and } \nabla \varphi(x) \in \Omega \text {. }
$$


Thanks to the regularity theory of the Monge-Ampère equation it is known that under some additional assumptions on the densities $f$ and $g$, the Brenier map $\nabla \varphi$ is defined everywhere on $\mathbb{R}^{n}$ and is a $\mathcal{C}^{1}$ diffeomorphism onto $\Omega$. In such case, the change of variable formula justifies that (3.12) is equivalent to the fact that $\varphi$ is a solution to the MongeAmpère equation

$$
\operatorname{det} \operatorname{Hess} \varphi(x)=\frac{f(x)}{g(\nabla \varphi(x))} .
$$

To this end we follow the argument of Caffarelli as presented in the paper [1].

First note that due to (3.17) the right hand side of (3.18) is defined $x$-a.e. Second, we use the result of Caffarelli [23] (see also Theorems 4.8 and 4.10 in [42]): since $\mu$ and $\nu$ are absolutely continuous with respect to the Lebesgue measure and the support of $\nu$ is convex, we have that $\varphi$ satisfies (3.18) in the Aleksandrov sense, i.e. the Hessian measure $\operatorname{det}_{H} \operatorname{Hess} \varphi$ associated to $\varphi$, defined by

$$
\operatorname{det}_{H} \operatorname{Hess} \varphi(A)=\operatorname{vol}_{n}(\partial \varphi(A)) \text { for any Borel set } A \subseteq \mathbb{R}^{n},
$$

is absolutely continuous with respect to the Lebesgue measure and its density coincides almost everywhere with the right-hand side of (3.18). For a proof of this result the following relation is crucial:

$$
\partial \varphi\left(\mathbb{R}^{n}\right) \subseteq \operatorname{cl} \Omega
$$

To see (3.19), use (3.16) and a general result on a subdifferential of a (closed) convex function [39, Theorem 25.6] which in our case says that for any $x \in \mathbb{R}^{n}, \partial \varphi(x)$ lies in the closure of the convex hull of limits of $\nabla \varphi\left(x_{k}\right)$, where $\left(x_{k}\right)$ runs through all sequences of points of differentiability of $\varphi$ which converge to $x$. Since $\operatorname{cl} \Omega$ is already convex and closed, $\partial \varphi(x) \subseteq \operatorname{cl} \Omega$ for any $x \in \mathbb{R}^{n}$.

Further on, we assume additionally that the functions $f$ and $1 / f$ are bounded on compact subsets of $\mathbb{R}^{n}$ and $g$ is bounded and bounded away from zero on $\Omega$. Then for any $R>0$ there exists $0<c(R)<C(R)<\infty$ such that the right hand side of (3.18) is between $c(R)$ and $C(R)$ almost everywhere in the ball $B(0, R)$. Since $\varphi$ satisfies (3.18) in the Aleksandrov sense, it follows that $\operatorname{det}_{H} \operatorname{Hess} \varphi$ has a density which is bounded and bounded away from zero on compact sets. With these a priori bounds on $\operatorname{det}_{H} \operatorname{Hess} \varphi$ we apply a geometric lemma of Caffarelli [22, Theorem 1] (see also [28, Chapter 5]) which will allow us to prove that $\varphi$ is strictly convex.

Lemma 3.8 (Caffarelli [22, Theorem 1]). Let $\Gamma \subseteq \mathbb{R}^{n}$ be an open bounded convex set and $\psi: \Gamma \rightarrow \mathbb{R}$ be a non-negative convex function. Suppose for some constants $0<c<C<\infty$,

$$
c \operatorname{vol}_{n}(A) \leq \operatorname{vol}_{n}(\partial \psi(A)) \leq C \operatorname{vol}_{n}(A) \text { for any Borel set } A \subseteq \Gamma .
$$

If the (convex) set $\{x \in \Gamma: \psi(x)=0\}$ is non-empty and contains more than one point, then it has no extremal points.

Corollary 3.9. $\varphi$ is strictly convex.

Proof. Assume the opposite. Then there exists $x_{0} \in \mathbb{R}^{n}$ and a supporting hyperplane $l$ of $\varphi$ at $x_{0}$ such the closed convex set that $F=\left\{x \in \mathbb{R}^{n}: \varphi(x)=l(x)\right\}$ contains some other point $z \neq x_{0}$.

First suppose that $F$ contains an extreme point, say $x_{1}$ (not necessarily distinct from $x_{0}$ ), and take $\Gamma$ to be an (open) ball $B(0, R)$ large enough to contain $x_{0}, z$ and the extreme point $x_{1}$. Let $\psi$ be the non-negative convex function $\varphi-l$ restricted to $\Gamma$. By translation invariance of the Lebesgue measure, $\operatorname{vol}_{n}(\partial \psi(A))=\operatorname{vol}_{n}(\partial \varphi(A))$ for any Borel set $A \subset \Gamma$ and thus we can apply Lemma 3.8. The set $\{x \in \Gamma: \psi(x)=0\}$ coincides with $F \cap \Gamma$ and since $F \cap \Gamma$ contains two distinct points $x_{0}$ and $z$, it follows from the lemma that 
$x_{1}$ cannot be an extreme point of $F \cap \Gamma$. This clearly contradicts the fact that $x_{1}$ is an extreme point of $F$.

Therefore $F$ is a non-empty closed convex set which has no extreme points. Hence it must contain a line (see e.g. [39, Corollary 18.5.3]). In consequence, the graph of $\varphi$ must contain a line and hence all hyperplanes supporting $\varphi$ are parallel to that line. This means that $\partial \varphi\left(\mathbb{R}^{n}\right)$ is contained in an affine subset of $\mathbb{R}^{n}$ of dimension at most $n-1$, which clearly contradicts (3.14).

Having established strict convexity of $\varphi$ we can conclude with a stronger statement than (3.19), namely that $\partial \varphi\left(\mathbb{R}^{n}\right)=\Omega$. It is based on a convexity argument.

Lemma 3.10. Let $\psi$ be a convex function on $\mathbb{R}^{n}$ with $\operatorname{dom} \psi=\mathbb{R}^{n}$. If $\psi$ is strictly convex then $\partial \psi\left(\mathbb{R}^{n}\right)=\operatorname{int} \operatorname{dom} \psi^{*}$.

Proof. In the view of (3.11) it is enough to prove that $\partial \psi\left(\mathbb{R}^{n}\right)$ is disjoint from bd dom $\psi^{*}$. To this end suppose $x \in \mathbb{R}^{n}$ and $y \in \partial \psi(x) \cap \operatorname{bd} \operatorname{dom} \psi^{*}$. Since $y \in \operatorname{bddom} \psi^{*}$, by convexity of $\operatorname{dom} \psi^{*}$ there exists $u \in \mathbb{R}^{n}$ such that

$$
\langle u, v-y\rangle \leq 0 \quad \text { for all } v \in \operatorname{dom} \psi^{*} .
$$

On the other hand, consider $z=x+\lambda u$ with any $\lambda>0$. By strict convexity of $\psi$,

$$
\begin{aligned}
& \psi(z)-\psi(x)>\langle z-x, y\rangle \\
& \psi(x)-\psi(z)>\langle x-z, v\rangle,
\end{aligned}
$$

where $v$ is an arbitrary vector from $\partial \psi(z)$. Adding up the two above inequalities yields $\langle z-x, y-v\rangle=\lambda\langle u, y-v\rangle<0$ which means that $\langle u, v-y\rangle>0$. Since by (3.11) the vector $v \in \partial \psi(z)$ belongs to dom $\psi^{*}$, it contradicts (3.20).

Applying the above lemma to $\varphi$ we get that $\partial \varphi\left(\mathbb{R}^{n}\right)$ is an open convex subset of $\mathbb{R}^{n}$. The fact that $\partial \varphi\left(\mathbb{R}^{n}\right)$ is open combined with (3.19) implies that $\partial \varphi\left(\mathbb{R}^{n}\right) \subseteq$ int $\operatorname{cl} \Omega=\Omega$. On the other hand, since $\partial \varphi\left(\mathbb{R}^{n}\right)$ is open and convex, then (3.14) yields $\Omega \subseteq \operatorname{int} \operatorname{cl} \partial \varphi\left(\mathbb{R}^{n}\right)=$ $\partial \varphi\left(\mathbb{R}^{n}\right)$. Therefore the three open convex sets

$$
\partial \varphi\left(\mathbb{R}^{n}\right)=\operatorname{int} \operatorname{dom} \varphi^{*}=\Omega
$$

coincide.

Finally, we use the following

Theorem 3.11 (Caffarelli [1, Theorem 1.3]). Let $\mu(d x)=f(x) d x$ and $\nu(d x)=g(x) d x$ be two probability measures on $\mathbb{R}^{n}$. Assume that $f$ is locally Hölder and strictly positive on $\mathbb{R}^{n}$. Assume that the restriction of $g$ to an open bounded convex set $\Omega$ is locally Hölder, bounded and bounded away from zero, and that $g \equiv 0$ in $\Omega^{c}$. Then any convex function $\varphi$ on $\mathbb{R}^{n}$ that induces the Brenier map $\nabla \varphi$ which pushes $\mu$ forward to $\nu$ belongs locally to the Hölder class $\mathcal{C}^{2, \alpha}$ for some $\alpha>0$ and satisfies (3.18) for all $x \in \mathbb{R}^{n}$.

We will also need a $\mathcal{C}^{2}$ convex function whose gradient pushes forward $\nu$ to $\mu$. Clearly, a natural candidate is $\varphi^{*}$. In the corollary below we state the final result we will use in the sequel.

Corollary 3.12. Assume $f$ and $g$ are as in Theorem 3.11.

(i) There exists a strictly convex function $\varphi \in \mathcal{C}^{2}\left(\mathbb{R}^{n}\right)$ with Hess $\varphi$ positive definite everywhere whose gradient $\nabla \varphi$ maps $\mathbb{R}^{n}$ onto $\Omega$, pushes $\mu$ forward to $\nu$ and thus satisfies the Monge-Ampère equation (3.18). 
(ii) For $\varphi$ as in (i), the Legendre conjugate $\varphi^{*}$ has int $\operatorname{dom} \varphi^{*}=\Omega$, belongs to $\mathcal{C}^{2}(\Omega)$, $\partial \varphi^{*}(y)=\emptyset$ for all $y \notin \Omega$. Moreover $\nabla \varphi^{*}$ pushes $\nu$ forward to $\mu$, Hess $\varphi^{*}$ is positive definite everywhere in $\Omega$ and $\varphi^{*}$ satisfies

$$
\operatorname{det} \operatorname{Hess} \varphi^{*}(y)=\frac{g(y)}{f\left(\nabla \varphi^{*}(y)\right)} \quad \text { for all } y \in \Omega .
$$

Proof. Consider $\varphi$ as in Theorem 3.11. In order to complete the proof of (i) it is enough to note that (3.21) becomes $\nabla \varphi\left(\mathbb{R}^{n}\right)=\operatorname{int} \operatorname{dom} \varphi^{*}=\Omega$.

(ii) Strict convexity of $\varphi$ allows us to conclude that $\nabla \varphi$ is a $\mathcal{C}^{1}$ bijection from $\mathbb{R}^{n}$ onto $\Omega$. Combining it with (3.10) shows that $\partial \varphi^{*}(y)=\left\{(\nabla \varphi)^{-1}(y)\right\}$ for $y \in \Omega$ and $\partial \varphi^{*}(y)=\emptyset$ for $y \notin \Omega$. The uniqueness of subgradient of $\varphi^{*}$ at each point of $\Omega$ implies that $\varphi^{*}$ is differentiable everywhere in int $\operatorname{dom} \varphi^{*}=\Omega$ and the map $\nabla \varphi^{*}: \Omega \rightarrow \mathbb{R}^{n}$ is the inverse map of $\nabla \varphi$. This is already sufficient to justify that $\nabla \varphi^{*}$ pushes $\nu$ forward to $\mu$. In order to show that $\varphi^{*}$ is in fact $\mathcal{C}^{2}(\Omega)$ and satisfies (3.22), it is enough to use that the Jacobian of the map $\nabla \varphi$ (i.e. $\operatorname{det} \operatorname{Hess} \varphi$ ) does not vanish and thus use the inverse function theorem to obtain that the map $\nabla \varphi^{*}$ is $\mathcal{C}^{1}(\Omega)$ and its derivative Hess $\varphi^{*}(y)$ equals (Hess $\left.\varphi(x)\right)^{-1}$ where $y \in \Omega$ and $x=\nabla \varphi^{*}(y) \in \mathbb{R}^{n}$. Thus (3.22) follows from (3.18).

3.5. Classes of test functions. For each $k=1,2, \ldots, m$ fix a measurable function $f_{k}: H_{k} \rightarrow[0, \infty]$ of integral one such that

- for $1 \leq i \leq m^{+}, f_{i}$ is locally Lipschitz, bounded and bounded away from zero on some bounded open convex subset of $H_{i}$, and vanishes outside this set;

- for $m^{+}<j \leq m, f_{j}$ is locally Lipschitz and strictly positive in its whole domain $H_{j}$.

The target functions are chosen as follows. Fix $R>0$ and consider any $m$-tuple $\left(A_{1}, \ldots, A_{m}\right) \in$ $\Lambda$ (for the definition of $\Lambda$ refer to Subsection 2.2) and also put $A_{0}=Q_{+}$and $A_{m+1}=Q_{-}$. For $k=0,1, \ldots, m+1$ define

$$
g_{k}(y)=\left(\operatorname{det} A_{k}\right)^{-1 / 2} \exp \left(-\pi\left\langle-A_{k}^{-1} y, y\right\rangle\right) .
$$

The target functions will be $\tilde{g}_{k}$ defined as

$$
\begin{aligned}
\tilde{g}_{i} & =g_{i} \quad \text { for } 1 \leq i \leq m^{+}, \\
\tilde{g}_{0} & =g_{0} \\
\tilde{g}_{m+1} & =g_{m+1}, \\
\tilde{g}_{j} & =\lambda_{j} g_{j} \mathbf{1}_{B_{H_{j}}(0, R)} \quad \text { for } m^{+}<j \leq m,
\end{aligned}
$$

where $\lambda_{j}>1$ is a normalizing constant (such that $\int_{H_{j}} \tilde{g}_{j}=1$ ).

3.6. Transportation argument. For each $i=1,2, \ldots, m^{+}$let $\varphi_{i}: H_{i} \rightarrow \mathbb{R} \cup\{+\infty\}$ be the function $\varphi^{*}$ from Corollary 3.12(ii) for probability measures $\mu$ and $\nu$ on $H_{i}$ having the densities $\tilde{g}_{i}$ and $f_{i}$ respectively. Each $\varphi_{i}$ belongs to $\mathcal{C}^{2}\left(\operatorname{int} \operatorname{dom} \varphi_{i}\right)$, is lower semi-continuous (as the Legendre transform of a convex function) and satisfies

$$
\partial \varphi_{i}(x)=\emptyset \quad \text { for all } x \notin \operatorname{int} \operatorname{dom} \varphi_{i} \text {. }
$$

For each $j=1+m^{+}, \ldots, m$ let $\varphi_{j}: H_{j} \rightarrow \mathbb{R}$ be the function $\varphi$ from Corollary 3.12(i) for probability measures $\mu$ and $\nu$ of $H_{j}$ having the densities $f_{j}$ and $\tilde{g}_{j}$ respectively. Each $\varphi_{j}$ is $\mathcal{C}^{2}\left(H_{j}\right)$ and

$$
\nabla \varphi_{j}(x) \in B_{H_{j}}(0, R) \quad \text { for all } x \in H_{j} .
$$

Additionally put $\varphi_{0}(x)=\frac{1}{2}\left\langle Q_{+} x, x\right\rangle$ and $\varphi_{m+1}(x)=\frac{1}{2}\left\langle Q_{-} x, x\right\rangle$. 
For $k=0,1,2, \ldots, m+1$, put $T_{k}=\nabla \varphi_{k}$ and note that by Corollary [3.12, for all $x \in \operatorname{int} \operatorname{dom} \varphi_{k}$,

$$
f_{k}(x)=\tilde{g}_{k}\left(T_{k}(x)\right) \operatorname{det} d T_{k}(x)
$$

and

$$
d T_{k}(x)=\operatorname{Hess} \varphi_{k}(x) \text { is symmetric positive definite }
$$

for all $x \in \operatorname{int} \operatorname{dom} \varphi_{k}$.

For $x \in H$ put

$$
\begin{aligned}
\varphi_{+}(x) & =\sum_{1 \leq i \leq m^{+}} c_{i} \varphi_{i}\left(B_{i} x\right), \\
\varphi_{-}(x) & =\sum_{m^{+}<j \leq m}\left(-c_{j}\right) \varphi_{j}\left(B_{j} x\right), \\
\varphi(x) & =\varphi_{0}\left(B_{0} x\right)+\varphi_{+}(x)-\varphi_{-}(x)-\varphi_{m+1}\left(B_{m+1} x\right)=\sum_{k=0}^{m+1} c_{k} \varphi_{k}\left(B_{k} x\right) .
\end{aligned}
$$

On the open domain $S=\bigcap_{i=1}^{m^{+}} B_{i}^{-1}\left(\operatorname{int} \operatorname{dom} \varphi_{i}\right) \subset H$, which is non-empty thanks to surjectivity of $B_{+}$, define the change of variable map $\theta: S \rightarrow H$ by

$$
\theta(x)=\nabla \varphi(x)=\sum_{k=0}^{m+1} c_{k} B_{k}^{*} T_{k}\left(B_{k} x\right) .
$$

This map is $\mathcal{C}^{1}$ and its differential equals

$$
\begin{aligned}
d \theta(x) & =\operatorname{Hess} \varphi(x)=\sum_{k=0}^{m+1} c_{k} B_{k}^{*} d T_{k}\left(B_{k} x\right) B_{k} \\
& =B_{0}^{*} Q_{+} B_{0}-B_{m+1}^{*} Q_{-} B_{m+1}+\sum_{k=1}^{m} c_{k} B_{k}^{*} d T_{k}\left(B_{k} x\right) B_{k} \\
& =Q+\sum_{k=1}^{m} c_{k} B_{k}^{*} d T_{k}\left(B_{k} x\right) B_{k} .
\end{aligned}
$$

Combining the above with (2.3) and (3.26) we obtain that for $x \in S$,

$$
\operatorname{det} d \theta(x) \leq D \prod_{k=1}^{m}\left(\operatorname{det} d T_{k}\left(B_{k}\right)\right)^{c_{k}}
$$

whenever $d \theta(x)$ is positive definite. Since (3.29) remains true for $d \theta(x)$ being positive semi-definite, we will consider the subdomain of $S$,

$$
S_{+}=\{x \in S: d \theta(x) \text { is positive semi-definite }\}
$$

on which (3.29) is valid. Note that by continuity of the map $S \ni x \mapsto d \theta(x), S_{+}$is a closed subset of $S$ and in particular $S_{+}$is a measurable subset of $H$.

As announced above, the following lemma is crucial in our argument. We defer its proof to Subsection 3.7.

Lemma 3.13. The map $\theta_{\mid S_{+}}: S_{+} \rightarrow H$ is surjective. 
Now we are in the position to establish a sharp lower bound on $J\left(f_{1}, \ldots, f_{m}\right)$. Starting from (3.9) and using the Monge-Ampère equations (3.25) we get

$$
\begin{aligned}
J\left(f_{1}, \ldots, f_{m}\right) & \geq \sqrt{\frac{\operatorname{det} Q_{-}}{\operatorname{det} Q_{+}}} \int_{S_{+}} \prod_{k=0}^{m+1} f_{k}^{c_{k}}\left(B_{k} x\right) d x \\
& \left.=\sqrt{\frac{\operatorname{det} Q_{-}}{\operatorname{det} Q_{+}}} \int_{S_{+}} \prod_{k=0}^{m+1}\left(\tilde{g}_{k}\left(T_{k}\left(B_{k} x\right)\right) \operatorname{det} d T_{k}\left(B_{k} x\right)\right)\right)^{c_{k}} d x \\
& =\sqrt{\frac{\operatorname{det} Q_{+}}{\operatorname{det} Q_{-}}} \int_{S_{+}}\left(\prod_{k=0}^{m+1} \tilde{g}_{k}^{c_{k}}\left(T_{k}\left(B_{k} x\right)\right)\right)\left(\prod_{k=1}^{m}\left(\operatorname{det} d T_{k}\left(B_{k} x\right)\right)^{c_{k}}\right) d x \\
& \geq D^{-1} \sqrt{\frac{\operatorname{det} Q_{+}}{\operatorname{det} Q_{-}}} \int_{S_{+}}\left(\prod_{k=0}^{m+1} \tilde{g}_{k}^{c_{k}}\left(T_{k}\left(B_{k} x\right)\right)\right) \operatorname{det} d \theta(x) d x \\
& =(*),
\end{aligned}
$$

where the latter inequality comes from (3.29). Setting $\lambda=\prod_{j=m^{+}+1}^{m} \lambda_{j}^{c_{j}}$ and using the point-wise estimate $\tilde{g}_{j}^{c_{j}} \geq \lambda_{j}^{c_{j}} g_{j}^{c_{j}}$ for $m^{+}<j \leq m$ we continue with the bound

$$
\begin{aligned}
(*) & \geq \lambda D^{-1} \sqrt{\frac{\operatorname{det} Q_{+}}{\operatorname{det} Q_{-}}} \int_{S_{+}}\left(\prod_{k=0}^{m+1} g_{k}^{c_{k}}\left(T_{k}\left(B_{k} x\right)\right)\right) \operatorname{det} d \theta(x) d x \\
& \geq \lambda D^{-1} \sqrt{\frac{\operatorname{det} Q_{+}}{\operatorname{det} Q_{-}}} \int_{S_{+}}\left(\inf _{\theta(x)=\sum_{k=0}^{m+1} c_{k} B_{k}^{*} y_{k}} \prod_{k=0}^{m+1} g_{k}^{c_{k}}\left(y_{k}\right)\right) \operatorname{det} d \theta(x) d x \\
& \geq \lambda D^{-1} \sqrt{\frac{\operatorname{det} Q_{+}}{\operatorname{det} Q_{-}}} \int_{H} \inf _{z=\sum_{k=0}^{m+1} c_{k} B_{k}^{*} y_{k}} \prod_{k=0}^{m+1} g_{k}^{c_{k}}\left(y_{k}\right) d z \\
& =\lambda D^{-1}\left(\prod_{k=1}^{m}\left(\operatorname{det} A_{k}\right)^{-c_{k} / 2}\right) \int_{H} \exp \left(-\pi \sup _{z=\sum_{k=0}^{m+1} c_{k} B_{k}^{*} y_{k}} \sum_{k=0}^{m+1} c_{k}\left\langle A_{k}^{-1} y_{k}, y_{k}\right\rangle\right) d z \\
& =(* *)
\end{aligned}
$$

where $\lambda=\prod_{j=m^{+}+1}^{m} \lambda_{j}^{c_{j}}$ and the last inequality follows from the area formula for $\mathcal{C}^{1}$ maps [26, Theorem 3.2.5] and the fact that the map $\theta \mid S_{+}: S_{+} \rightarrow H$ is surjective (Lemma 3.13). Using Lemma 3.5, we finish the above estimate with

$$
\begin{aligned}
(* *) & =\lambda D^{-1}\left(\prod_{k=1}^{m}\left(\operatorname{det} A_{k}\right)^{-c_{k} / 2}\right) \int_{H} \exp \left(-\pi\left\langle\left(\sum_{k=0}^{m+1} c_{k} B_{k}^{*} A_{k} B_{k}\right)^{-1} z, z\right\rangle\right) d z \\
& =\lambda D^{-1}\left(\frac{\operatorname{det}\left(Q+\sum_{k=1}^{m} c_{k} B_{k}^{*} A_{k} B_{k}\right)}{\prod_{k=1}^{m}\left(\operatorname{det} A_{k}\right)^{c_{k}}}\right)^{1 / 2} .
\end{aligned}
$$

Thanks to (2.3) , taking supremum over $\left(A_{k}\right)_{k=1}^{m} \in \Lambda$ and the limit $R \rightarrow \infty$ (which results in $\lambda \uparrow 1)$ yields the desired inequality, i.e. $J\left(f_{1}, \ldots, f_{m}\right) \geq D^{-1 / 2}$.

\subsection{Surjectivity of the map $\theta$.}

Lemma 3.14. Let $f: H \rightarrow \mathbb{R} \cup\{+\infty\}$ be convex, lower semi-continuous and $g: H \rightarrow \mathbb{R}$ be convex. Assume that $\operatorname{dom} f \neq \emptyset$ and $\partial f(x)=\emptyset$ at every $x \in \operatorname{bd} \operatorname{dom} f$. If $f(x)-g(x) \rightarrow$ $+\infty$ as $|x| \rightarrow+\infty$ then $f-g$ attains its infimum at a point in $\operatorname{int} \operatorname{dom} f$. 
Proof. Note that $f-g$ is lower semi-continuous which combined with the hypothesis that $f-g \rightarrow+\infty$ at infinity implies that the sets $A_{r}=\{x \in H: f(x)-g(x) \leq r\}$ are compact for all $r \in \mathbb{R}$. Since $\operatorname{dom} f \neq \emptyset, A_{r}$ is non-empty for $r$ large enough. Therefore $f-g$ attains its infimum at some point $x \in \operatorname{dom} f$. Suppose $x \in \operatorname{bd} \operatorname{dom} f$. Take any $x^{*} \in \partial g(x)$ (note that the subdifferential of $g$ is everywhere non-empty). By hypothesis, $\partial f(x)=\emptyset$ hence we can find $y \in H$ such that

$$
f(y)<f(x)+\left\langle x^{*}, y-x\right\rangle .
$$

On the other hand we have

$$
g(y) \geq g(x)+\left\langle x^{*}, y-x\right\rangle,
$$

which combined with (3.31) gives

$$
f(y)-g(y)<f(x)-g(x)=\inf (f-g)
$$

and hence contradicts the assumption that $x \in \operatorname{bd} \operatorname{dom} f$.

We will need one more lemma, about the function $\varphi$, defined in (3.27).

Lemma 3.15. The function $\varphi$ is superlinear, i.e.

$$
\lim _{|x| \rightarrow \infty} \frac{\varphi(x)}{|x|}=+\infty
$$

Proof. Consider the compact set

$$
F=\prod_{i=1}^{m^{+}} \operatorname{cl} \operatorname{dom} \varphi_{i}
$$

Obviously

$$
\operatorname{dom} \varphi_{+}=\bigcap_{i=1}^{m^{+}} B_{i}^{-1}\left(\operatorname{dom} \varphi_{i}\right) \subseteq B_{+}^{-1}(F)
$$

Using (3.5) we get

$$
\sup _{x \in \operatorname{dom} \varphi_{+}} \varphi_{m+1}\left(B_{m+1} x\right)=C_{1}<\infty .
$$

For each $1 \leq i \leq m^{+}$, since $\operatorname{dom} \varphi_{i}$ is bounded, we have $\inf _{H_{i}} \varphi_{i}>-\infty$ and thus $\inf _{H} \varphi_{+}>-\infty$. Therefore for some constant $C_{2}<\infty$ we have

$$
\varphi_{+}(x) \geq\left|B_{+} x\right|^{2}-C_{2}
$$

for all $x \in H$ (it is enough to ensure this inequality on the set $B_{+}^{-1}(F)$, inside which $\left|B_{+} x\right|^{2}-C_{2}$ has finite supremum).

Combining (3.33) with the fact that $\varphi_{0}$ is a positive definite quadratic function on $H_{0}$, we get

$$
\varphi_{0}\left(B_{0} x\right)+\varphi_{+}(x) \geq \varepsilon\left|B_{0} x\right|^{2}+\left|B_{+} x\right|^{2}-C_{2} \geq \varepsilon^{\prime}|x|^{2}-C_{2},
$$

for some $\varepsilon, \varepsilon^{\prime}>0$, where in the last inequality we used injectivity of the map $B_{0+}=$ $\left(B_{0}, B_{+}\right)$. Combining the above estimate with (3.32) we get

$$
\varphi(x)+\varphi_{-}(x)=\varphi_{0}\left(B_{0} x\right)+\varphi_{+}(x)-\varphi_{m+1}\left(B_{m+1} x\right) \geq \varepsilon^{\prime}|x|^{2}-C_{1}-C_{2},
$$

hence the function $\varphi+\varphi_{-}$is superlinear.

Due to (3.24) the function $\varphi_{-}$is Lipschitz. Therefore $\varphi$ is also superlinear.

Now we are ready to establish our claim about the map $\theta$. 
Proof of Lemma 3.13. Consider the function $f: H \rightarrow \mathbb{R} \cup\{+\infty\}$ defined by

$$
f(x)=\varphi_{0}\left(B_{0} x\right)+\varphi_{+}(x)=\sum_{i=0}^{m^{+}} c_{i} \varphi_{i}\left(B_{i} x\right) .
$$

Clearly $f$ is convex and lower semi-continuous. Note also that

$$
\operatorname{dom} f=\bigcap_{1 \leq i \leq m^{+}} \operatorname{dom}\left(\varphi_{i} \circ B_{i}\right)=\bigcap_{1 \leq i \leq m^{+}} B_{i}^{-1}\left(\operatorname{dom} \varphi_{i}\right)
$$

and

$$
\operatorname{int} \operatorname{dom} f=\bigcap_{1 \leq i \leq m^{+}} B_{i}^{-1}\left(\operatorname{int} \operatorname{dom} \varphi_{i}\right),
$$

i.e. int dom $f$ coincides with the domain $S$.

Using Theorems 23.8 and 23.9 from [39] we express the subdifferential of $f$ in terms of subdifferentials of $\varphi_{0}$ and $\varphi_{i}$ with $1 \leq i \leq m^{+}$. Namely for all $x \in H$ we have

$$
\partial f(x)=\sum_{0 \leq i \leq m^{+}} c_{i} B_{i}^{*} \partial \varphi_{i}\left(B_{i} x\right)
$$

where the summation means the Minkowski sum of sets (in $H$ ). (The above formula is an equality rather than merely an inclusion $\supset$ if e.g. the set $\bigcap_{i=0}^{m^{+}} \operatorname{dom}\left(\varphi_{i} \circ B_{i}\right) \neq \emptyset$; this follows from the fact that the domain $S$ is non-empty). Combining the above with (3.23) we obtain that if $x \notin S$ (i.e. for some $\left.1 \leq i \leq m^{+}, B_{i} x \notin \operatorname{int} \operatorname{dom} \varphi_{i}\right)$ then $\partial f(x)=\emptyset$.

Fix any $y_{0} \in H$. We claim that the $\mathcal{C}^{2}$ function $S \ni x \mapsto \varphi(x)-\left\langle x, y_{0}\right\rangle$ attains a local minimum at, say, $x_{0} \in S$. This will allow to establish the lemma. Indeed, since $S$ is an open set, the gradient of this function vanishes at $x_{0}$, i.e. $\nabla \varphi\left(x_{0}\right)-y_{0}=0$ and $\operatorname{Hess} \varphi\left(x_{0}\right)$ is positive semi-definite, which means that $\theta\left(x_{0}\right)=y_{0}$ and $x_{0} \in S_{+}$.

Eventually, let us prove the claim that $\varphi(\cdot)-\left\langle\cdot, y_{0}\right\rangle: S \rightarrow H$ attains its infimum. Beside the function $f$ defined in (3.34), consider a convex function $g: H \rightarrow \mathbb{R}$,

$$
g(x)=\varphi_{-}(x)+\varphi_{m+1}\left(B_{m+1} x\right)+\left\langle x, y_{0}\right\rangle .
$$

Obviously

$$
f(x)-g(x)=\varphi(x)-\left\langle x, y_{0}\right\rangle
$$

By Lemma 3.15 and the fact that $\left\langle\cdot, y_{0}\right\rangle$ is Lipschitz we obtain that $f-g$ is superlinear at infinity, so in particular $f(x)-g(x) \rightarrow \infty$ as $|x| \rightarrow \infty$. Since the subdifferential of $f$ is empty outside $S=\operatorname{int} \operatorname{dom} f$, we can use Lemma 3.14 in order to conclude.

3.8. Approximation argument. For non-negative, integrable functions $f_{i}(1 \leq i \leq$ $\left.m^{+}\right)$and $f_{j}\left(m^{+}<j \leq m\right)$ denote

$$
\mathcal{I}\left(\left(f_{i}\right),\left(f_{j}\right)\right)(x)=e^{-\mathcal{Q}_{+}\left(B_{0} x\right)} e^{\mathcal{Q}_{-}\left(B_{m+1} x\right)} \prod_{i=1}^{m^{+}} f_{i}^{c_{i}}\left(B_{i} x\right) \prod_{j=1+m^{+}}^{m} f_{j}^{c_{j}}\left(B_{j} x\right) \quad \text { for } x \in H .
$$

We proved that under the hypothesis of Theorem 2.9,

$$
\int_{H} \mathcal{I}\left(\left(f_{i}\right),\left(f_{j}\right)\right) \geq K \prod_{1 \leq i \leq m^{+}}\left(\int_{H_{i}} f_{i}\right)^{c_{i}} \prod_{m^{+}<j \leq m}\left(\int_{H_{j}} f_{j}\right)^{c_{j}}
$$

for all $f_{i} \in \mathcal{F}_{i}^{0}\left(1 \leq i \leq m^{+}\right)$and for all $f_{j} \in \mathcal{F}_{j}^{0}\left(m^{+}<j \leq m\right)$, where $K=\inf _{\mathcal{C G}} J$ and

- $\mathcal{F}_{i}^{0}$ is the class of non-negative functions on $H_{i}$ which are locally Lipschitz, bounded and bounded away from zero on an open bounded convex subset of $H_{i}$, and vanish outside this set,

- $\mathcal{F}_{j}^{0}$ is the class of strictly positive and locally Lipschitz functions on $H_{j}$. 
We proceed in three steps $s=1,2,3$. In each step we consider different classes of functions $\mathcal{F}_{i}^{s}$ and $\mathcal{F}_{j}^{s}$ for which we prove (3.36) to be valid. At the final step $s=3$, the classes $\mathcal{F}_{i}^{3}$, $\mathcal{F}_{j}^{3}$ will consist of all non-negative, integrable functions.

Step 1. Fix $f_{i} \in \mathcal{F}_{i}^{1}\left(1 \leq i \leq m^{+}\right)$and $f_{j} \in \mathcal{F}_{j}^{1}\left(m^{+}<j \leq m\right)$ where

- $\mathcal{F}_{i}^{1}$ is the class of non-negative bounded measurable functions on $H_{i}$ with compact support,

- $\mathcal{F}_{j}^{1}$ is the class of positive bounded Lipschitz functions $f_{j}$ on $H_{j}$ for which $f_{j}(y)^{-1}$ is bounded from above by a polynomial in $|y|$.

Note that $f_{j}$ belongs to $\mathcal{F}_{j}^{0}$ as well. For each $i$, take $R_{i}>0$ such that the ball $B_{H_{i}}\left(0, R_{i}\right)$ contains the support of $f_{i}$. Consider the sequence of functions

$$
f_{i, n}=f_{i} * \phi_{i, n}+\frac{1}{n} \mathbf{1}_{B_{H_{i}}\left(0, R_{i}+1\right)},
$$

where $\phi_{i, n}(x)=c_{i} n^{\operatorname{dim} H_{i}} \operatorname{dist}\left(x, H_{i} \backslash B_{H_{i}}(0,1 / n)\right)$ and $c_{i}$ is such that $\int_{H_{i}} \phi_{i, n}=1$.

Since $\phi_{i, n}$ are bounded, Lipschitz and $f_{i}$ are non-negative and measurable with compact support, $f_{i, n} \in \mathcal{F}_{i}^{0}$. Moreover $\int_{H_{i}} f_{i, n} \rightarrow \int_{H_{i}} f_{i}$ and by the Lebesgue differentiation theorem, $f_{i, n} \rightarrow f_{i}$ a.e.. In other words the set $\Omega_{i} \subset H_{i}$ where the latter convergence holds has a negligible complement. Then the convergence $\mathcal{I}\left(\left(f_{i, n}\right),\left(f_{j}\right)\right) \rightarrow \mathcal{I}\left(\left(f_{i}\right),\left(f_{j}\right)\right)$ holds for all points in the set $\Omega:=\bigcap_{i=1}^{m^{+}} B_{i}^{-1}\left(\Omega_{i}\right)$. Since the maps $B_{i}$ are surjective, the complement of $\Omega$ is negligible. Hence $\mathcal{I}\left(\left(f_{i, n}\right),\left(f_{j}\right)\right) \rightarrow \mathcal{I}\left(\left(f_{i}\right),\left(f_{j}\right)\right)$ a.e.

In order to verify (3.36) it is enough to ensure that

$$
\lim _{n \rightarrow \infty} \int_{H} \mathcal{I}\left(\left(f_{i, n}\right),\left(f_{j}\right)\right)=\int_{H} \mathcal{I}\left(\left(f_{i}\right),\left(f_{j}\right)\right) .
$$

To this end we find an integrable function on $H$ which dominates $\mathcal{I}\left(\left(f_{i, n}\right),\left(f_{j}\right)\right)$ uniformly in $n$ and then apply the Lebesgue dominated convergence theorem.

First, since $f_{i}$ are bounded, all $f_{i, n}$ are bounded uniformly in $n$ and thus for some constant $C>0$ and a compact set $F \subseteq H_{1} \times \cdots \times H_{m^{+}}$,

$$
\prod_{i=1}^{m^{+}} f_{i, n}^{c_{i}}\left(B_{i} x\right) \leq C \mathbf{1}_{F}\left(B_{+} x\right) \quad \text { for all } x \in H
$$

Second, by (3.5) $), B_{m+1}\left(B_{+}^{-1}(F)\right)$ is compact, hence after adjusting the constant $C$, we also have

$$
e^{\mathcal{Q}_{-}\left(B_{m+1} x\right)} \prod_{i=1}^{m^{+}} f_{i, n}^{c_{i}}\left(B_{i} x\right) \leq C \mathbf{1}_{F}\left(B_{+} x\right) \quad \text { for all } x \in H .
$$

Since the map $\left(B_{0}, B_{+}\right)$is a linear isomorphism, $\mathcal{I}\left(\left(f_{i, n}\right),\left(f_{j}\right)\right)$ would be compactly supported if only the function $e^{-\mathcal{Q}_{+}}$was compactly supported. Obviously it is not (unless $\mathcal{Q}_{+}$is trivial), but we can still use a compactness argument by decomposing $e^{-\mathcal{Q}_{+}}$into slices, namely

$$
\begin{aligned}
e^{-\mathcal{Q}_{+}(y)} & =\int_{0}^{1} \mathbf{1}_{\left\{e^{-\mathcal{Q}_{+}} \geq u\right\}}(y) d u=\int_{0}^{\infty} 2 t e^{-t^{2}} \mathbf{1}_{\left\{\mathcal{Q}_{+} \leq t^{2}\right\}}(y) d t \\
& =\int_{0}^{\infty} 2 t e^{-t^{2}} \mathbf{1}_{t\left\{\mathcal{Q}_{+} \leq 1\right\}}(y) d t .
\end{aligned}
$$


Combining the above with (3.38), we can bound $\mathcal{I}\left(\left(f_{i, n}\right),\left(f_{j}\right)\right)(x)$ pointwise and uniformly in $n$ by a constant times

$$
\begin{array}{r}
\int_{0}^{\infty} t e^{-t^{2}} \mathbf{1}_{t\left\{\mathcal{Q}_{+} \leq 1\right\}}\left(B_{0} x\right) \mathbf{1}_{F}\left(B_{+} x\right) d t \prod_{j=1+m^{+}}^{m} f_{j}^{c_{j}}\left(B_{j} x\right) \\
\leq \int_{0}^{\infty} t e^{-t^{2}} \mathbf{1}_{(t+1) B_{H}(0, R)}(x) d t \prod_{j=1+m^{+}}^{m} f_{j}^{c_{j}}\left(B_{j} x\right)
\end{array}
$$

with $R>0$ large enough. Since $f_{j}(y)^{-1}$ is bounded from above by a polynomial in $|y|$, we finally obtain a pointwise upper bound

$$
\mathcal{I}\left(\left(f_{i, n}\right),\left(f_{j}\right)\right)(x) \leq \int_{0}^{\infty}\left(C_{1} t^{\alpha}+C_{2}\right) e^{-t^{2}} \mathbf{1}_{(t+1) B_{H}(0, R)}(x) d t
$$

with some constants $C_{1}, C_{2}, \alpha>0$, which is clearly an integrable function of $x \in H$.

Step 2. Fix $f_{i} \in \mathcal{F}_{i}^{2}\left(1 \leq i \leq m^{+}\right)$and $f_{j} \in \mathcal{F}_{j}^{2}\left(m^{+}<j \leq m\right)$ where

- $\mathcal{F}_{i}^{2}=\mathcal{F}_{i}^{1}$

- $\mathcal{F}_{j}^{2}$ is the class of non-negative integrable functions on $H_{j}$.

Let $\phi(u)=\frac{1}{\pi\left(1+u^{2}\right)}$ be the density of the standard Cauchy distribution and for each $j=1+m^{+}, \ldots, m$ and $\lambda>0$ put

$$
\phi_{j, \lambda}(x)=\lambda^{\operatorname{dim} H_{j}} \prod_{l=1}^{\operatorname{dim} H_{j}} \phi\left(\lambda x_{l}\right) .
$$

Fix $\varepsilon>0$ and for each $j$ and $n$ put

$$
f_{j, n}=\left(f_{j}+\varepsilon \phi_{j, 1}\right) * \phi_{j, n} .
$$

For each $j$ and $n, \phi_{j, n}$ is bounded and Lipschitz and $f_{j}$ is integrable, hence $f_{j, n}$ is also bounded and Lipschitz. From the estimate

$$
f_{j, n} \geq \varepsilon \phi_{j, 1} * \phi_{j, n}=\varepsilon \phi_{j, \frac{n}{n+1}} \geq \varepsilon 2^{-\operatorname{dim} H_{j}} \phi_{j, 1}
$$

(the equality above follows from the fact that the Cauchy distribution is 1-stable), we obtain that

$$
f_{j, n}(y)^{-1} \text { is bounded from above by a polynomial in }|y| \text { uniformly in } n \text {. }
$$

Hence we proved that $f_{j, n} \in \mathcal{F}_{j}^{1}$.

By the result of Step 1 (i.e. (3.36) for $\left(f_{i}\right),\left(f_{j, n}\right)$ ),

$$
\begin{aligned}
\int_{H} \mathcal{I}\left(\left(f_{i}\right),\left(f_{j, n}\right)\right) & \geq K \prod_{1 \leq i \leq m^{+}}\left(\int_{H_{i}} f_{i}\right)^{c_{i}} \prod_{m^{+}<j \leq m}\left(\int_{H_{j}} f_{j, n}\right)^{c_{j}} \\
& =K \prod_{1 \leq i \leq m^{+}}\left(\int_{H_{i}} f_{i}\right)^{c_{i}} \prod_{m^{+}<j \leq m}\left(\varepsilon+\int_{H_{j}} f_{j}\right)^{c_{j}},
\end{aligned}
$$

where the last equality follows from $\int_{H_{j}} f_{j, n}=\varepsilon+\int_{H_{j}} f_{j}$. Obviously

$$
\int_{H} \mathcal{I}\left(\left(f_{i}\right),\left(f_{j}\right)\right) \geq \int_{H} \mathcal{I}\left(\left(f_{i}\right),\left(f_{j}+\varepsilon \phi_{j, 1}\right)\right)
$$

so proving

$$
\lim _{n \rightarrow \infty} \int_{H} \mathcal{I}\left(\left(f_{i}\right),\left(f_{j, n}\right)\right)=\int_{H} \mathcal{I}\left(\left(f_{i}\right),\left(f_{j}+\varepsilon \phi_{j, 1}\right)\right)
$$


would yield

$$
\int_{H} \mathcal{I}\left(\left(f_{i}\right),\left(f_{j}\right)\right) \geq K \prod_{1 \leq i \leq m^{+}}\left(\int_{H_{i}} f_{i}\right)^{c_{i}} \prod_{m^{+}<j \leq m}\left(\int_{H_{j}} f_{j}+\varepsilon\right)^{c_{j}}
$$

and in consequence (3.36) by letting $\varepsilon \rightarrow 0$.

Since $f_{j, n} \rightarrow f_{j}+\varepsilon \phi_{j, 1}$ a.e., we have $\mathcal{I}\left(\left(f_{i}\right),\left(f_{j, n}\right)\right) \rightarrow \mathcal{I}\left(\left(f_{i}\right),\left(f_{j}+\varepsilon \phi_{j, 1}\right)\right)$ a.e. In the view of (3.39) and the fact that $f_{i}$ are bounded with compact support, we can proceed as in Step 1 to find an integrable function on $H$ which dominates $\mathcal{I}\left(\left(f_{i}\right),\left(f_{j, n}\right)\right)$ for all $n$ and conclude with (3.40).

Step 3. Fix $f_{i} \in \mathcal{F}_{i}^{3}\left(1 \leq i \leq m^{+}\right)$and $f_{j} \in \mathcal{F}_{j}^{3}\left(m^{+}<j \leq m\right)$ where

- $\mathcal{F}_{i}^{3}$ is the class of non-negative integrable functions on $H_{i}$,

- $\mathcal{F}_{j}^{3}=\mathcal{F}_{j}^{2}$.

We approximate $f_{i}$ with $f_{i, n}=\min \left(f_{i}, n\right) \mathbf{1}_{B_{H_{i}}(0, n)}$ which belong to $\mathcal{F}_{i}^{2}$. The convergence as in (3.37) follows from the monotone convergence theorem. We conclude with (3.36) by using the result of Step 2 for the functions $\left(f_{i, n}\right),\left(f_{j}\right)$.

\section{Geometric Brascamp-Lieb inequality}

We study specific non-degenerate situations for which inf $J=1$ and some extremizing functions can be identified. They are related to geometric Brascamp-Lieb inequalities and the decomposition of the identity (1.1). More precisely, they are characterized by the following conditions:

$$
\begin{aligned}
B_{k} B_{k}^{*} & =\operatorname{Id}_{H_{k}} \quad \text { for } k=1, \ldots, m, \\
Q+\sum_{k=1}^{m} c_{k} B_{k}^{*} B_{k} & =\operatorname{Id}_{H} .
\end{aligned}
$$

4.1. Finding $\inf _{\mathcal{C G}} J$. The aim of this subsection is to prove that if the non-degeneracy condition (1.4) and the geometric conditions (4.1) and (4.2) hold then the infimum of $J$ on centered Gaussian functions is equal to 1 , and is achieved when for all $k, f_{k}(\cdot)=$ $\exp \left(-\pi|\cdot|^{2}\right)$. The crucial result here is Proposition 4.4 which establishes a concavity property of a function related to Formula (2.2).

First, let us put forward two useful facts and a lemma.

Fact 4.1 (see e.g. [29, Theorem 7.7.6]). Let $A$ be $n \times n$ real symmetric matrix and $C$ be $m \times m$ real symmetric matrix and $B$ be an $n \times m$ real matrix. Let $X=\left(\begin{array}{cc}A & B \\ B^{*} & C\end{array}\right)$. If $C>0$ then

(i) $X \geq 0$ if and only if $A-B C^{-1} B^{*} \geq 0$;

(ii) $X>0$ if and only if $A-B C^{-1} B^{*}>0$.

Below $\mathrm{I}_{n}$ denotes the $n \times n$ identity matrix.

Fact 4.2 (Woodbury formula). For an $m \times n$ matrix $A$,

$$
A^{*}\left(\mathrm{I}_{m}+A A^{*}\right)^{-1} A=\mathrm{I}_{n}-\left(\mathrm{I}_{n}+A^{*} A\right)^{-1} \text {. }
$$

Proof. Direct calculation.

Lemma 4.3. Let $R$ be a $p \times n$ real matrix and $S$ be an $r \times n$ real matrix. Consider the $(p+r) \times(p+r)$ matrix

$$
M=\left(\begin{array}{cc}
-\mathrm{I}_{p}+R R^{*} & R S^{*} \\
S R^{*} & \mathrm{I}_{r}+S S^{*}
\end{array}\right)
$$


(i) $M \geq 0$ if and only if $R\left(\mathrm{I}_{n}+S^{*} S\right)^{-1} R^{*} \geq \mathrm{I}_{p}$.

(ii) If $n=p$ and $R$ is invertible then $M \geq 0$ if and only if $R^{*} R-S^{*} S \geq \mathrm{I}_{n}$.

Proof. Applying Fact 4.1(i) we obtain that $M \geq 0$ is equivalent to

$$
-\mathrm{I}_{p}+R R^{*}-R S^{*}\left(\mathrm{I}_{r}+S S^{*}\right)^{-1} S R^{*} \geq 0 .
$$

Using Fact 4.2 for $A=S$ the above can be rephrased as

$$
-\mathrm{I}_{p}+R R^{*}-R\left(\mathrm{I}_{n}-\left(\mathrm{I}_{n}+S^{*} S\right)^{-1}\right) R^{*} \geq 0,
$$

which finishes the proof of (i).

If $n=p$ and $R$ is invertible, then $M \geq 0$ is also equivalent to

$$
\left(\mathrm{I}_{n}+S^{*} S\right)^{-1} \geq R^{-1} R^{-*}=\left(R^{*} R\right)^{-1}
$$

which in turn is equivalent to

$$
\mathrm{I}_{n}+S^{*} S \leq R^{*} R
$$

In the context of Brascamp-Lieb inequalities the following easy consequence of the Cauchy-Binet formula is useful, see Proposition 6 of [11]: if $d \leq n$ and $U$ is an $n \times d$ matrix, then the map

$$
x \in \mathbb{R}^{n} \mapsto \log \operatorname{det}\left(U^{*} \operatorname{diag}\left(\left(e^{x_{i}}\right)_{i \leq n}\right) U\right)
$$

is convex. The next property is a counterpart for inverse Brascamp-Lieb inequalities.

Proposition 4.4. Let $m \geq n \geq 1$ and $U$ be an invertible $n \times n$ real matrix and $V$ be a real $(m-n) \times n$ matrix. Let

$$
\Omega=\left\{\left(x_{1}, \ldots, x_{m}\right) \in \mathbb{R}^{m}: U^{*} \operatorname{diag}\left(\left(e^{x_{i}}\right)_{i \leq n}\right) U-V^{*} \operatorname{diag}\left(\left(e^{x_{j}}\right)_{j>n}\right) V>0\right\} .
$$

Then $\Omega$ is convex and the map $\phi: \Omega \rightarrow \mathbb{R}$,

$$
\phi\left(x_{1}, \ldots, x_{m}\right)=\log \operatorname{det}\left(U^{*} \operatorname{diag}\left(\left(e^{x_{i}}\right)_{i \leq n}\right) U-V^{*} \operatorname{diag}\left(\left(e^{x_{j}}\right)_{j>n}\right) V\right)
$$

is concave.

Proof. First we show that $\Omega$ is convex. Take any $x=\left(x_{1}, \ldots, x_{m}\right) \in \Omega, y=\left(y_{1}, \ldots, y_{m}\right) \in$ $\Omega$ and $\lambda \in(0,1)$. Let $X=\operatorname{diag}\left(\left(e^{x_{k}} / 2\right)_{1 \leq k \leq m}\right), Y=\operatorname{diag}\left(\left(e^{y_{k} / 2}\right)_{1 \leq k \leq m}\right)$ and $X_{+}, X_{-}$be the diagonal blocks of $X$ of size $n \times n$ and $(m-n) \times(m-n)$ (resp.), similarly $Y_{+}, Y_{-}$.

From $x \in \Omega$ it follows that $U^{*} X_{+}^{2} U>V^{*} X_{-}^{2} V$. By invertibility of $U$,

$$
\mathrm{I}_{n}>X_{+}^{-1} U^{-*} V^{*} X_{-}^{2} V U^{-1} X_{+}^{-1}=\left(X_{-} V U^{-1} X_{+}^{-1}\right)^{*}\left(X_{-} V U^{-1} X_{+}^{-1}\right)
$$

which is equivalent to $\left\|X_{-} V U^{-1} X_{+}^{-1}\right\|<1(\|\cdot\|$ denotes the operator norm). Similarly, $y \in S$ implies $\left\|Y_{-} V U^{-1} Y_{+}^{-1}\right\|<1$.

Put $A=Y_{-} V U^{-1} X_{+}^{-1}$ and $B=X_{-} Y_{-}^{-1}$ and $C=X_{+} Y_{+}^{-1}$. Then we have

$$
\|B A\|<1, \quad\|A C\|<1 \text {. }
$$

Now use [17, Corollary IX.5.3] which asserts that

$$
\left\|B^{\lambda} A C^{1-\lambda}\right\| \leq\|B A\|^{\lambda}\|A C\|^{1-\lambda}
$$

to obtain

$$
\mathrm{I}_{n}>\left(B^{\lambda} A C^{1-\lambda}\right)^{*}\left(B^{\lambda} A C^{1-\lambda}\right)=Y_{+}^{\lambda-1} X_{+}^{-\lambda} U^{-*} V^{*} X_{-}^{2 \lambda} Y_{-}^{2(1-\lambda)} V U^{-1} X_{+}^{-\lambda} Y_{+}^{\lambda-1},
$$

which is equivalent to

$$
U^{*}\left(X_{+}^{2}\right)^{\lambda}\left(Y_{+}^{2}\right)^{1-\lambda} U>V^{*}\left(X_{-}^{2}\right)^{\lambda}\left(Y_{-}^{2}\right)^{1-\lambda} V
$$

Since $\left(X^{2}\right)^{\lambda}\left(Y^{2}\right)^{1-\lambda}$ is the diagonal matrix with the entries $e^{\lambda x_{k}+(1-\lambda) y_{k}}$, (4.3) ensures that $\lambda x+(1-\lambda) y \in \Omega$. 
Next we establish concavity of $\phi$ on $\Omega$. For $x=\left(x_{1}, \ldots, x_{m}\right) \in \mathbb{R}^{m}$, set $x_{+}=$ $\left(x_{1}, \ldots, x_{n}\right) \in \mathbb{R}^{n}$ and $x_{-}=\left(x_{n+1}, \ldots, x_{m}\right) \in \mathbb{R}^{m-n}$. Let $A: \mathbb{R}^{m} \rightarrow \mathbb{R}^{n \times n}$ be defined as

$$
A(x)=U^{*} e^{\operatorname{diag}\left(x_{+}\right)} U-V^{*} e^{\operatorname{diag}\left(x_{-}\right)} V .
$$

Then $\phi(x)=\log \operatorname{det} A(x)$ for $x \in \Omega$. Since $\phi$ is a smooth function, we can analyze the Hessian of $\phi$. To this end, we will use the following formulas:

$$
\begin{aligned}
\partial \log \operatorname{det} X & =\operatorname{tr}\left(X^{-1} \partial X\right), \quad \text { for } X>0 \\
\partial X^{-1} & =-X^{-1}(\partial X) X^{-1}, \quad \text { for } X>0 \\
\partial A(x) & =U^{*} e^{\operatorname{diag}\left(x_{+}\right)} \operatorname{diag}\left((\partial x)_{+}\right) U-V^{*} e^{\operatorname{diag}\left(x_{-}\right)} \operatorname{diag}\left((\partial x)_{-}\right) V .
\end{aligned}
$$

Specialization of (4.6) to partial derivatives gives

$$
\begin{aligned}
& \partial_{i} A(x)=e^{x_{i}} U^{*} e_{i} e_{i}^{*} U \quad \text { for } i \leq n, \\
& \partial_{j} A(x)=-e^{x_{j}} V^{*} f_{j-n} f_{j-n}^{*} V \quad \text { for } j>n,
\end{aligned}
$$

where for $i \leq n, e_{i}$ is a column matrix with $n$ rows, a coefficient 1 in the $i$-th row and all other coefficients equal to 0 . Similarly, for $\ell \leq m-n, f_{\ell}$ is a column matrix with $m-n$ rows, with a 1 in its $\ell$-th row and zeroes elsewhere. Fix $x \in \Omega$. Using (4.4) and (4.7), for $i \leq n$ we obtain

$$
\partial_{i} \phi(x)=\operatorname{tr}\left(A^{-1}(x) \partial_{i} A(x)\right)=e^{x_{i}} e_{i}^{*} U A^{-1}(x) U^{*} e_{i},
$$

Similarly, for $j>n$,

$$
\partial_{j} \phi(x)=-e^{x_{j}} e_{j-n}^{*} V A^{-1}(x) V^{*} e_{j-n} .
$$

In order to calculate second order partial derivatives, we use (4.5) combined with (4.7) or (4.8). For $i_{1}, i_{2} \leq n$ and $i_{1} \neq i_{2}$ we have

$$
\begin{aligned}
\partial_{i_{1} i_{2}}^{2} \phi(x) & =-e^{x_{i_{1}}} e_{i_{1}}^{*} U A^{-1}(x) \partial_{i_{2}} A(x) A^{-1} U^{*} e_{i_{1}} \\
& =-e^{x_{i_{1}}+x_{i_{2}}} e_{i_{1}}^{*} U A^{-1}(x) U^{*} e_{i_{2}} e_{i_{2}}^{*} U A^{-1}(x) U^{*} e_{i_{1}}
\end{aligned}
$$

Denoting

$$
R=e^{\operatorname{diag}\left(x_{+}\right) / 2} U A^{-1 / 2}(x)
$$

we can write the above second order mixed partial derivative in a more compact way

$$
\partial_{i_{1} i_{2}}^{2} \phi(x)=-\left(R R^{*}\right)_{i_{1} i_{2}}\left(R R^{*}\right)_{i_{2} i_{1}}=-\left(R R^{*}\right)_{i_{1} i_{2}}^{2},
$$

and for $i \leq n$ we have

$$
\partial_{i i}^{2} \phi(x)=\partial_{i} \phi(x)-\left(R R^{*}\right)_{i i}^{2}=\left(R R^{*}\right)_{i i}-\left(R R^{*}\right)_{i i}^{2} .
$$

Combining the two above formulas we can write that for any $i_{1}, i_{2} \leq n$,

$$
\partial_{i_{1} i_{2}}^{2} \phi(x)=\left(R R^{*}\right)_{i_{1} i_{2}}\left(\mathrm{I}_{n}-R R^{*}\right)_{i_{1} i_{2}} .
$$

If we denote

$$
S=e^{\operatorname{diag}\left(x_{-}\right) / 2} V A^{-1 / 2}(x),
$$

then by similar calculations we get that for $j_{1}, j_{2}>n$,

$$
\partial_{j_{1}, j_{2}}^{2} \phi(x)=-\left(S S^{*}\right)_{j_{1}-n, j_{2}-n}\left(\mathrm{I}_{m-n}+S S^{*}\right)_{j_{1}-n, j_{2}-n} .
$$

Lastly, for $i \leq n$ and $j>n$,

$$
\begin{aligned}
\partial_{i j}^{2} \phi(x) & =-e^{x_{i}} e_{i}^{*} U A^{-1}(x) \partial_{j} A(x) A^{-1}(x) U^{*} e_{i} \\
& =e^{x_{i}+x_{j}} e_{i}^{*} U A^{-1}(x) V^{*} f_{j-n} f_{j-n}^{*} V A^{-1}(x) U^{*} e_{i} \\
& =\left(R S^{*}\right)_{i, j-n}\left(S R^{*}\right)_{j-n, i}=\left(R S^{*}\right)_{i, j-n}^{2}=\left(S R^{*}\right)_{j-n, i}^{2}
\end{aligned}
$$


As a result,

$$
\begin{aligned}
\operatorname{Hess} \phi(x) & =-\left(\begin{array}{cc}
\left(R R^{*}\right) \circ\left(-\mathrm{I}_{n}+R R^{*}\right) & -\left(R S^{*}\right) \circ\left(R S^{*}\right) \\
-\left(S R^{*}\right) \circ\left(S R^{*}\right) & \left(S S^{*}\right) \circ\left(\mathrm{I}_{m-n}+S S^{*}\right)
\end{array}\right) \\
& =-\underbrace{\left(\begin{array}{cc}
R R^{*} & -R S^{*} \\
-S R^{*} & S S^{*}
\end{array}\right)}_{M} \underbrace{\left(\begin{array}{cc}
-\mathrm{I}_{n}+R R^{*} & R S^{*} \\
S R^{*} & \mathrm{I}_{m-n}+S S^{*}
\end{array}\right)}_{N},
\end{aligned}
$$

where $A \circ B$ denotes the Hadamard product (i.e. entry-wise product) of $A$ and $B$.

Note that $M$ is positive semi-definite. Indeed, $M=\left(\begin{array}{c}R \\ -S\end{array}\right)\left(R^{*},-S^{*}\right) \geq 0$. Now we argue that also $N \geq 0$. From definitions of the matrices $A(x)$ and $R$ and $S$ it follows immediately that

$$
R^{*} R-S^{*} S=\mathrm{I}_{n}
$$

Since $U$ is invertible, so does $R$ and Lemma 4.3(ii) implies $N \geq 0$.

Now it is enough to apply the Schur product theorem (see e.g. [29, Theorem 7.5.3]) which asserts that if $M$ and $N$ are positive semi-definite then $M \circ N$ is also positive semi-definite. Therefore Hess $\phi(x)$ is negative semi-definite at any $x \in \Omega$ and hence $\phi$ is concave.

The next theorem uses the notation from Subsection 2.2.

Theorem 4.5. Assume the non-degeneracy condition (1.4) holds. Let $\left(A_{1}, \ldots, A_{m}\right) \in$ $\Lambda$. Put $A=Q+\sum_{k=1}^{m} c_{k} B_{k}^{*} A_{k} B_{k}>0$. Then the supremum in (2.3) is attained at $\left(A_{1}, \ldots, A_{m}\right)$, i.e.

$$
D=\frac{\operatorname{det} A}{\prod_{i=k}^{m}\left(\operatorname{det} A_{k}\right)^{c_{k}}}
$$

if and only if

$$
A_{k}^{-1}-B_{k} A^{-1} B_{k}^{*}=0 \quad \text { for all } k=1, \ldots, m \text { for which } c_{k} \neq 0 .
$$

In particular, if (4.1) and (4.2) hold then $D=1$.

Remark 4.6. If (4.10) holds, then $\tilde{Q}:=A^{-1 / 2} Q A^{-1 / 2}$ and $\tilde{B}_{k}:=A_{k}^{1 / 2} B_{k} A^{-1 / 2}$ satisfy the generalized geometric conditions (4.1) and (4.2). This allows to show that up to linear isomorphisms (by $A^{1 / 2}$ on $H$ and $A_{k}^{1 / 2}$ on $H_{k}$ ) the situations where $\inf _{\mathcal{C G}} J$ is achieved are equivalent to the geometric situations. This follows exactly what happens for direct Brascamp-Lieb inequalities, see [15].

Proof of Theorem 4.5. Consider the function $\Phi: \Lambda \rightarrow \mathbb{R}$,

$$
\Phi\left(M_{1}, \ldots, M_{m}\right)=\log \operatorname{det}\left(Q+\sum_{k=1}^{m} c_{k} B_{k}^{*} M_{k} B_{k}\right)-\sum_{k=1}^{m} c_{k} \log \operatorname{det} M_{k}
$$

Note $\Phi$ is smooth and $\sup _{\Lambda} \Phi=\log D$. 
Fix any self-adjoint operators $X_{k}: H_{k} \rightarrow H_{k}$ (for $k=1, \ldots, m$ ). Using Formula (4.4) the directional derivative of $\Phi$ at $\left(A_{1}, \ldots, A_{m}\right)$ in the direction of $\left(X_{1}, \ldots, X_{m}\right)$ is

$$
\begin{aligned}
& \partial_{\left(X_{1}, \ldots, X_{m}\right)} \Phi\left(A_{1}, \ldots, A_{m}\right) \\
& =\lim _{t \rightarrow 0} \frac{1}{t}\left(\Phi\left(A_{1}+t X_{1}, \ldots, A_{m}+t X_{m}\right)-\Phi\left(A_{1}, \ldots, A_{m}\right)\right) \\
& =\operatorname{tr}\left(A^{-1}\left(\sum_{k=1}^{m} c_{k} B_{k}^{*} X_{k} B_{k}\right)\right)-\sum_{k=1}^{m} c_{k} \operatorname{tr}\left(A_{k}^{-1} X_{k}\right) \\
& =\sum_{k=1}^{m} c_{k} \operatorname{tr}\left(A^{-1} B_{k}^{*} X_{k} B_{k}\right)-\sum_{k=1}^{m} c_{k} \operatorname{tr}\left(A_{k}^{-1} X_{k}\right)=\sum_{k=1}^{m} c_{k} \operatorname{tr}\left(\left(B_{k} A^{-1} B_{k}^{*}-A_{k}^{-1}\right) X_{k}\right) .
\end{aligned}
$$

The condition (4.9) implies that the derivative must be 0 . Using the fact that a self-adjoint operator $Y$ is zero if and only if $\operatorname{tr}(Y X)=0$ for all self-adjoint operators $X$, (4.10) follows by considering $X_{1}, \ldots, X_{k-1}, X_{k+1}, \ldots, X_{m}$ being zero and $X_{k}$ being arbitrary for each $k=1, \ldots, m$ such that $c_{k} \neq 0$.

For the converse implication assume that (4.10) holds. Then the above calculation shows that the derivative of $\Phi$ at $\left(A_{1}, \ldots, A_{m}\right)$ is zero. In order to conclude that $\Phi$ has a global maximum at this point, we prove below that $\Phi$ enjoys a concavity type property along well chosen curves.

Fix any self-adjoint operators $Y_{k}: H_{k} \rightarrow H_{k}$ (for $k=1, \ldots, m$ ) and for any real $t$ put

$$
\mathbf{A}(t)=\left(A_{1}^{1 / 2} \exp \left(t Y_{1}\right) A_{1}^{1 / 2}, \ldots, A_{m}^{1 / 2} \exp \left(t Y_{m}\right) A_{m}^{1 / 2}\right) .
$$

For $t \in \mathbb{R}$ for which $\mathbf{A}(t) \in \Lambda$ consider the function

$$
\varphi(t)=\Phi(\mathbf{A}(t)) .
$$

Since $\Lambda$ is an open set and the function $t \mapsto \mathbf{A}(t)$ is continuous, the domain of $\varphi$ is an open subset of $\mathbb{R}$. The domain contains 0 and, as $\varphi$ is smooth, $\varphi^{\prime}(0)$ vanishes.

For each $k=1, \ldots, m$ take an orthogonal transformation $U_{k} \in O\left(H_{k}\right)$ such that $U_{k}^{*} Y_{k} U_{k}$, when identified with its matrix in the standard basis $\left(e_{l}^{H_{k}}\right)_{l}$ in $H_{k}$, is a diagonal matrix. Denote the diagonal entries by $y_{k 1}, \ldots, y_{k n_{k}}$, where $n_{k}=\operatorname{dim} H_{k}$. Then

$$
B_{k}^{*} A_{k}^{1 / 2} \exp \left(t Y_{k}\right) A_{k}^{1 / 2} B_{k}=\left(U_{k} A_{k}^{1 / 2} B_{k}\right)^{*} \operatorname{diag}\left(\left(e^{t y_{k l}}\right)_{l \leq n_{k}}\right) U_{k} A_{k}^{1 / 2} B_{k} .
$$

Thanks for the non-degeneracy condition (1.4) we can use the decomposition of the Gaussian kernel $\exp (-\mathcal{Q})$ as asserted by Lemma 3.1. Beside the maps $B_{0}: H \rightarrow H_{0}$ and $B_{m+1}: H \rightarrow H_{m+1}$ consider also $A_{0}>0$ on $H_{0}$ and $A_{m+1}>0$ on $H_{m+1}$ such that

$$
Q=\sum_{k \in\{0, m+1\}} c_{k} B_{k}^{*} A_{k} B_{k}
$$

with $c_{0}=1$ and $c_{m+1}=-1$. For the sake of consistency with (4.12), for $k \in\{0, m+1\}$ put $Y_{k}=0$ (a zero map on $H_{k}$ ), $U_{k}=\operatorname{Id}_{H_{k}}$ and $y_{k l}=0$ for all $l \leq n_{k}=\operatorname{dim} H_{k}$.

Let

$$
\begin{aligned}
U & =\left(\sqrt{c_{i}} U_{i} A_{i}^{1 / 2} B_{i}\right)_{0 \leq i \leq m^{+}}: H \rightarrow H_{0} \times \cdots \times H_{m^{+}} \\
V & =\left(\sqrt{-c_{j}} U_{j} A_{j}^{1 / 2} B_{j}\right)_{m^{+}<j \leq m++1}: H \rightarrow H_{m^{+}+1} \times \cdots \times H_{m+1} .
\end{aligned}
$$

Considering the diagonal matrix $D_{+}(t)=\operatorname{diag}\left(\left(e^{t y_{i l}}\right)_{0 \leq i \leq m^{+}, l \leq n_{i}}\right)$ as an operator acting on $H_{0} \times \cdots \times H_{m^{+}}$and the diagonal matrix $D_{-}(t)=\operatorname{diag}\left(\left(e^{t y_{j l}}\right)_{m^{+}<j \leq m+1, l \leq n_{j}}\right)$ as an operator acting on $H_{m^{+}+1} \times \cdots \times H_{m+1}$ we can write

$$
\varphi(t)=\log \operatorname{det}\left(U^{*} D_{+}(t) U-V^{*} D_{-}(t) V\right)-\sum_{k=1}^{m} c_{k} \log \operatorname{det} A_{k}-t \sum_{k=1}^{m} c_{k} \operatorname{tr} Y_{k},
$$


where used the formula $\log \operatorname{det}(\exp (Y))=\operatorname{tr} Y$ for a self-adjoint $Y$.

It follows from Assertion (3.1) that $U$ is a linear isomorphism. Therefore we can apply Proposition 4.4, which tells us that the domain of $\varphi$ must be an open interval and that $\varphi$ is concave. Since $\varphi^{\prime}(0)=0, \varphi$ attains its global maximum at $t=0$.

Since for any $\left(X_{1}, \ldots, X_{m}\right) \in \Lambda$ there exist $t$ and self adjoint operators $Y_{k}$ on $H_{k}$ (for $k=1, \ldots, m)$ such that $\left(X_{1}, \ldots, X_{m}\right)$ is of the form (4.11) (e.g. take $t=1$ and $Y_{k}=\log \left(A_{k}^{-1 / 2} X_{k} A_{k}^{-1 / 2}\right)$, we actually showed that $\left(A_{1}, \ldots, A_{m}\right)$ is a global maximum of $\Phi$ and thus (4.9) holds.

\subsection{Geometric version of Inverse Brascamp-Lieb inequalities.}

Theorem 4.7. For $k=1, \ldots, m$, let $c_{k} \in \mathbb{R}$ and let $B_{k}: H \rightarrow H_{k}$ be linear surjective maps such that $B_{k} B_{k}^{*}=\operatorname{Id} H_{k}$. Let $Q: H \rightarrow H$ be a symmetric operator. Assume that

$$
Q+\sum_{k=1}^{m} c_{k} B_{k}^{*} B_{k}=\operatorname{Id}_{H} \quad \text { and } \quad \operatorname{dim} H \geq s^{+}(Q)+\sum_{k: c_{k}>0} \operatorname{dim} H_{k} .
$$

Then for all non-negative integrable functions $h_{k}: H_{k} \rightarrow[0,+\infty]$ with $\int h_{k}>0$, it holds

$$
\int_{H} \exp (-\pi\langle x, Q x\rangle) \prod_{k=1}^{m} h_{k}^{c_{k}}\left(B_{k} x\right) d x \geq \prod_{k=1}^{m}\left(\int_{H_{k}} h_{k}\right)^{c_{k}} .
$$

There is equality when for all $k$ and all $y \in H_{k}, f_{k}(y)=\exp \left(-\pi|y|^{2}\right)$.

Proof. We may assume without loss of generality that $c_{1}, \ldots, c_{m^{+}}>0>c_{1+m^{+}}, \ldots, c_{m}$. The above decomposition of the identity implies that

$$
Q+\sum_{i=1}^{m^{+}} c_{i} B_{i}^{*} B_{i}=\operatorname{Id}_{H}+\sum_{j>m^{+}}\left|c_{j}\right| B_{j}^{*} B_{j}>0 .
$$

Hence the restriction of $Q$ to $\operatorname{ker} B_{+}=\bigcap_{i=1}^{m^{+}} \operatorname{ker} B_{i}$ is positive definite. The non-degeneracy conditions (1.4) are verified and we may apply Theorem 2.9 to conclude inf $J=\inf _{\mathcal{C G}} J$. Then Theorem 4.5 ensures that $\inf _{\mathcal{C G}} J=D^{-\frac{1}{2}}=1$.

4.3. Relation with the results of Chen, Dafnis and Paouris. The reverse Gaussian correlation inequality by Chen, Dafnis and Paouris, presented here in Theorem 1.3, turns out to be the geometric version of our main result. To see this, let us consider a slight reformulation of the second inequality from Theorem [1.3, which appears explicitly in [25]:

Theorem 4.8 ([25, Theorem 3(ii)]). Let $\gamma_{E}$ stand for the standard Gaussian measure on a Euclidean space $E$. Let $B_{k}: H \rightarrow H_{k}$ (for $k=1, \ldots, m$ ) be linear maps satisfying $B_{k} B_{k}^{*}=\operatorname{Id}_{H_{k}}$. Denote

$$
B=\left(B_{1}, \ldots, B_{m}\right): H \rightarrow H_{1} \times \cdots \times H_{m}
$$

and let $C: H_{1} \times \cdots \times H_{m} \rightarrow H_{1} \times \cdots \times H_{m}$ be the block diagonal operator defined as

$$
C=\operatorname{diag}\left(c_{1} \operatorname{Id}_{H_{1}}, \ldots, c_{m} \operatorname{Id}_{H_{m}}\right) .
$$

If

$$
B B^{*} \geq C^{-1}
$$

then for any non-negative functions $f_{k} \in L^{1}\left(H_{k}, \gamma_{H_{k}}\right)(k=1, \ldots, m)$,

$$
\int_{H} \prod_{k=1}^{m} f_{k}^{c_{k}}\left(B_{k} x\right) d \gamma_{H}(x) \geq \prod_{k=1}^{m}\left(\int_{H_{k}} f_{k} d \gamma_{H_{k}}\right)^{c_{k}},
$$


By setting $h_{k}(x)=f_{k}(\sqrt{2 \pi} x) e^{-\pi|x|^{2}}$ we can rewrite the above inequality in terms of integrals with respect to the Lebesgue measure:

$$
\int_{H} \exp (-\pi\langle x, Q x\rangle) \prod_{k=1}^{m} h_{k}^{c_{k}}\left(B_{k} x\right) d x \geq \prod_{k=1}^{m}\left(\int_{H_{k}} h_{k}\right)^{c_{k}},
$$

where $Q=\operatorname{Id}_{H}-\sum_{k=1}^{m} c_{k} B_{k}^{*} B_{k}$. Hence the geometric condition (4.2) is obviously satisfied. In order to deduce Theorem 4.8 from Theorem 4.7, we need to establish the dimension condition $\operatorname{dim} H \geq s^{+}(Q)+\sum_{k: c_{k}>0} \operatorname{dim} H_{k}$. This is what we do next.

Assume as usual that $c_{1}, \ldots, c_{m^{+}}>0$ and $c_{m^{+}+1}, \ldots, c_{m}<0$. Recall that $B_{+}=$ $\left(B_{1}, \ldots, B_{m^{+}}\right)$and set $B_{-}=\left(B_{m^{+}+1}, \ldots, B_{m}\right)$ and $H_{+}=H_{1} \times \cdots \times H_{m^{+}}, H_{-}=H_{m^{+}+1} \times$ $\cdots \times H_{m}$. The condition (4.13) is equivalent to

$$
|C|^{1 / 2} B B^{*}|C|^{1 / 2} \geq\left(\begin{array}{ll}
\operatorname{Id}_{H_{+}} & \\
& -\operatorname{Id}_{H_{-}}
\end{array}\right) .
$$

Introducing $\tilde{B}_{k}=\left|c_{k}\right|^{1 / 2} B_{k}$ for $k=1, \ldots, m$ and defining $\tilde{B}_{+}$and $\tilde{B}_{-}$correspondingly, the above condition can be rewritten as

$$
\left(\begin{array}{cc}
-\operatorname{Id}_{H_{+}}+\tilde{B}_{+} \tilde{B}_{+}^{*} & \tilde{B}_{+} \tilde{B}_{-}^{*} \\
\tilde{B}_{-} \tilde{B}_{+}^{*} & \operatorname{Id}_{H_{-}}+\tilde{B}_{-} \tilde{B}_{-}^{*}
\end{array}\right) \geq 0 .
$$

Since the upper-left corner of the above matrix is semi-definite positive, we know that $\tilde{B}_{+} \tilde{B}_{+}^{*}$ is positive definite and hence $\tilde{B}_{+}$is surjective, so does $B_{+}$. Moreover, from Lemma 4.3(i) we get that (4.14) is equivalent to

$$
\tilde{B}_{+}\left(\operatorname{Id}_{H}+\tilde{B}_{-}^{*} \tilde{B}_{-}\right)^{-1} \tilde{B}_{+}^{*} \geq \operatorname{Id}_{H_{+}},
$$

which in view of the identity $Q=\operatorname{Id}_{H}-\tilde{B}_{+}^{*} \tilde{B}_{+}+\tilde{B}_{-}^{*} \tilde{B}_{-}$implies

$$
\tilde{B}_{+}^{*} \tilde{B}_{+}\left(\tilde{B}_{+}^{*} \tilde{B}_{+}+Q\right)^{-1} \tilde{B}_{+}^{*} \tilde{B}_{+} \geq \tilde{B}_{+}^{*} \tilde{B}_{+} .
$$

Thanks to the lemma below we conclude that $s^{+}(Q) \leq \operatorname{dim}$ ker $\tilde{B}_{+}$, which coincides with the dimension condition (2.5) since $\operatorname{dim} \operatorname{ker} \tilde{B}_{+}=\operatorname{dim} H-\operatorname{dim} H_{+}$due to surjectivity of $\tilde{B}_{+}$.

Lemma 4.9. Let $A, B$ be real symmetric matrices of size $d$ such that $A \geq 0$ and $A+B>0$. If $A(A+B)^{-1} A \geq A$ then $s^{+}(B) \leq \operatorname{dim} \operatorname{ker} A$.

Proof. Observe that the statement of this lemma is invariant under congruency (i.e. under replacing $A$ with $C^{*} A C$ and $B$ with $C^{*} B C$ ). Since $A+B>0$ there exists an invertible matrix such that $C^{*}(A+B) C$ and $C^{*} A C$ are both diagonal. By subtraction we get that $C^{*} B C$ is diagonal too. Hence, we may assume without loss of generality that $A=$ $\operatorname{diag}\left(\left(a_{i}\right)_{i=1}^{d}\right)$ and $B=\operatorname{diag}\left(\left(b_{i}\right)_{i=1}^{d}\right)$ with for all $i, a_{i} \geq 0$ and $a_{i}+b_{i}>0$. The hypothesis $A(A+B)^{-1} A \geq A$ reads as $a_{i}^{2} /\left(a_{i}+b_{i}\right) \geq a_{i}$, which is equivalent to $0 \geq a_{i} b_{i}$, for all $i$. Since $a_{i} \geq 0$, we may deduce that for all $i$

$$
b_{i}>0 \Longrightarrow a_{i}=0 .
$$

The matrices being diagonal, this implication means that $s^{+}(B) \leq \operatorname{dim} \operatorname{ker} A$.

Let us also comment on the Lebesgue version of the inverse Brascamp-Lieb inequalities presented in [25] as Theorem 2(ii). Applying suitable linear transformation in the Euclidean spaces $H$ and $H_{1}, \ldots, H_{m}$ one can formulate that result as follows: 
Theorem 4.10 ([25, Theorem 2(ii)]). In the settings of Theorem 4.8, if

$$
\operatorname{dim} H=\sum_{k=1}^{m} c_{k} \operatorname{dim} H_{k}
$$

and $B B^{*} \geq C^{-1}$ then for any non-negative integrable functions $f_{k} \in H_{k} \rightarrow[0, \infty)$,

$$
\int_{H} \prod_{k=1}^{m} f_{k}^{c_{k}}\left(B_{k} x\right) d x \geq \prod_{k=1}^{m}\left(\int_{H_{k}} f_{k}\right)^{c_{k}} .
$$

Let us explain how to reprove this results from what we already did, and settle a question on existence of cases of equalities that was left open in [25]. Recall that $B B^{*} \geq C^{-1}$ is equivalent to (4.14) and implies that $B_{+}$(equivalently $\tilde{B}_{+}$) is surjective. There are two possible cases:

Case 1: $B_{+}$(equivalently $\tilde{B_{+}}$) is injective. In this case we can apply Lemma 4.3(ii) to get that (4.14) is equivalent to $\tilde{B}_{+}^{*} \tilde{B}_{+}-\tilde{B}_{-}^{*} \tilde{B}_{-} \geq \operatorname{Id}_{H}$ or simply

$$
\sum_{k=1}^{m} c_{k} B_{k}^{*} B_{k} \geq \operatorname{Id}_{H}
$$

Using the $B_{k} B_{k}^{*}=\operatorname{Id}_{H_{k}}$ and (4.15) we see the maps on both sides of the above inequality have the same trace. Hence there must be equality in (4.17), i.e. the geometric condition (4.2) holds. In particular for the functions $f_{k}(x)=\exp \left(-\pi|x|^{2}\right)$ we get equality in (4.16). The decomposition of the identity also allows to deduce (4.16) from Theorem 4.13 applied to the functions $f_{k}(\cdot) \exp \left(|\cdot|^{2} / 2\right)$.

Case 2: $B_{+}$has a non-trivial kernel. Since $B_{+}$is surjective and $Q=0$, we are in the degenerate case 0.1 of the case analysis made in Subsection 2.5, in which the lefthand side of (4.16) is always infinite and thus (4.16) does not admit extremizers.

\section{Dual Form of inVerse Brascamp-Lieb inequalities}

The transportation technique that we have used in Section 3 in order to prove inverse Brascamp-Lieb inequalities follows the one used by the first named author in [11]. In this reference, the method is actually proved to establish two inequalities:

- The classical multilinear Brascamp-Lieb inequality, of the form

$$
\int_{H} \prod_{i=0}^{m} f_{i}\left(B_{i} x\right)^{c_{i}} d x \leq C_{B L} \prod_{i=0}^{m}\left(\int_{H_{i}} f_{i}\right)^{c_{i}},
$$

- The "dual" Brascamp-Lieb inequality,

$$
\int_{H}^{*} \sup _{\sum_{i} c_{i} B_{i}^{*} x_{i}=x} \prod_{i=0}^{m} f_{i}\left(x_{i}\right)^{c_{i}} d x \geq C_{D B L} \prod_{i=0}^{m}\left(\int_{H_{i}} f_{i}\right)^{c_{i}} .
$$

For both inequalities, the optimal constant is obtained by inspecting centered Gaussian functions. Also the only relevant indices are $c_{i} \in(0,1]$. Moreover it is possible to introduce a kernel by fixing $c_{0}=1$ and $f_{0}$ to be a specific Gaussian functions, and then to consider the best constant for arbitrary non-negative integrable functions $f_{1}, \ldots, f_{m}$.

Let us reproduce the proof of Theorem 2.9 of the inverse Brascamp Lieb inequality

$$
\int_{H} \prod_{i=0}^{m+1} f_{i}\left(B_{i} x\right)^{c_{i}} d x \geq C_{I B L} \prod_{i=0}^{m+1}\left(\int_{H_{i}} f_{i}\right)^{c_{i}}
$$

but choosing the functions $g_{1}, \ldots, g_{m}$ to be arbitrary (we omit here to repeat the regularity and support assumptions, that can be achieved by approximation. Recall that $c_{0}=1=$ 
$-c_{m+1}$ and that $f_{0}, g_{0}, f_{m+1}, g_{m+1}$ are specific Gaussian functions, which model Gaussian kernels). With our notation,

$$
J\left(f_{1}, \ldots, f_{m}\right)=\frac{\int_{H} e^{-\pi\left\langle Q_{+} B_{0} x, B_{0} x\right\rangle+\pi\left\langle Q_{-} B_{m+1} x, B_{m+1} x\right\rangle} \prod_{k=1}^{m} f_{k}\left(B_{k} x\right)^{c_{k}} d x}{\prod_{k=1}^{m}\left(\int_{H_{k}} f_{k}\right)^{c_{k}}} .
$$

In view of (3.30) we also set (* standing for inner integral):

$$
K\left(g_{1}, \ldots, g_{m}\right)=\frac{\int_{*, H} \inf _{\sum c_{k} B_{k}^{*} y_{k}=y} e^{-\pi\left\langle Q_{+}^{-1} y_{0}, y_{0}\right\rangle+\pi\left\langle Q_{-}^{-1} y_{m+1}, y_{m+1}\right\rangle} \prod_{k=1}^{m} g_{k}\left(y_{k}\right)^{c_{k}} d y}{\prod_{k=1}^{m}\left(\int_{H_{k}} g_{k}\right)^{c_{k}}}
$$

The above transportation argument, up to (3.30) yields $J\left(f_{1}, \ldots, f_{m}\right) \geq D^{-1} K\left(g_{1}, \ldots, g_{m}\right)$ for all functions, hence inf $J \geq D^{-1} \sup K$. However, we have seen in (2.3) that $\inf _{\mathcal{C G}} J=$ $D^{-\frac{1}{2}}$. The conclusion of the argument after (3.30) can be rephrased as $\sup _{\mathcal{C G}} K=D^{\frac{1}{2}}$. Therefore

$$
\sqrt{D}=D \inf _{\mathcal{C G}} J \geq D \inf J \geq \sup K \geq \sup _{\mathcal{C G}} K=\sqrt{D}
$$

In particular $\sup K=\sup _{\mathcal{C G}} K$. This means that under the non-degeneracy hypothesis of Theorem 2.9, the best constant in the following inequality (which can be called dual inverse Brascamp-Lieb) is obtained by inspecting centered Gaussian functions only: for all $g_{1}, \ldots, g_{m}$,

$$
\int_{*, H} \inf _{c_{k} B_{k}^{*} y_{k}=y} \prod_{k=0}^{m+1} g_{k}\left(y_{k}\right)^{c_{k}} d y \leq C_{D I B L} \prod_{k=1}^{m}\left(\int_{H_{k}} g_{k}\right)^{c_{k}} .
$$

Let us state the simplest examples of these four inequalities: no kernel, two functions, all maps being the identity: for all $f, g: \mathbb{R}^{n} \rightarrow \mathbb{R}^{+}$with $\int f \in(0,+\infty)$ :

If $\lambda \in(0,1)$,

$$
\int f(x)^{\lambda} g(x)^{1-\lambda} d x \leq\left(\int f\right)^{\lambda}\left(\int g\right)^{1-\lambda} \leq \int_{\lambda a+(1-\lambda) b=x}^{*} \sup _{\lambda(a)^{\lambda} g(b)^{1-\lambda}} d x
$$

If $\lambda \in \mathbb{R} \backslash[0,1]$,

$$
\int f(x)^{\lambda} g(x)^{1-\lambda} d x \geq\left(\int f\right)^{\lambda}\left(\int g\right)^{1-\lambda} \geq \int_{*} \inf _{\lambda a+(1-\lambda) b=x} f(a)^{\lambda} g(b)^{1-\lambda} d x
$$

The reader has recognized the inequalities of Hölder, Prékopa-Leindler and the inverse Hölder inequality. The fourth inequality seems novel. In this very simple situation, the inequalities for $\lambda \in \mathbb{R} \backslash[0,1]$ can be deduced from the ones for $\lambda \in(0,1)$ by rearranging the terms.

\section{INTERPOLATION}

We have proved that the best constant in inverse Brascamp-Lieb inequalities can be computed using centered Gaussian functions, apart from some degenerate situations. In the rest of the paper, we address the question of positivity of this optimal constant. More precisely, given a quadratic form $\mathcal{Q}$ and the geometric data $B=\left(B_{k}\right)_{k=1}^{m}$, our aim is to characterize exponents $c=(c)_{k=1}^{m}$ for which a non-trivial inverse Brascamp-Lieb inequality holds, meaning $\inf J_{\mathcal{Q}, B, c}>0$ where

$$
J_{\mathcal{Q}, B, c}\left(f_{1}, \ldots, f_{m}\right)=\frac{\int_{H} e^{-\mathcal{Q}(x)} \prod_{i=k}^{m} f_{k}^{c_{k}}\left(B_{k} x\right) d x}{\prod_{k=1}^{m}\left(\int_{H_{k}} f_{k}\right)^{c_{k}}} .
$$


The analogous question for direct Brascamp-Lieb inequality was solved in full generality by Bennett, Carbery, Christ and Tao [15, 16]. They gave a description of the set $\mathcal{F}$ of exponents $c$ for which $\sup J_{\mathcal{Q}, B, c}<+\infty$. It turns out that this set $\mathcal{F}$ is convex, which is a simple instance of interpolation of Lebesgue spaces. Actually, this may be proved by mere application of the Cauchy-Schwarz inequality: if $t \in[0,1]$,

$$
\begin{aligned}
\int e^{-\mathcal{Q}} \prod_{k=1}^{m} f_{k}^{t c_{k}+(1-t) d_{k}} \circ B_{k} & \leq\left(\int e^{-\mathcal{Q}} \prod_{k=1}^{m} f_{k}^{c_{k}} \circ B_{k}\right)^{t}\left(\int e^{-\mathcal{Q}} \prod_{k=1}^{m} f_{k}^{d_{k}} \circ B_{k}\right)^{1-t} \\
& \leq\left(\sup J_{\mathcal{Q}, B, c}\right)^{t}\left(\sup J_{\mathcal{Q}, B, d}\right)^{1-t} \prod_{k=1}^{m}\left(\int_{H_{k}} f_{k}\right)^{t c_{k}+(1-t) d_{k}} .
\end{aligned}
$$

In the setting of inverse inequalities, we did not find a simple interpolation argument as above. Nevertheless the convexity of the set of non-trivial exponents is still valid, provided one prescribes their signs.

Proposition 6.1. Let $0 \leq m^{+} \leq m$, linear surjective maps $B_{k}: H \rightarrow H_{k}, 1 \leq k \leq m$ and a quadratic form $\mathcal{Q}: H \rightarrow \mathbb{R}$. Assume that $\mathcal{Q}$ is positive definite on ker $B_{+}$and

$$
\operatorname{dim} H \geq s^{+}(\mathcal{Q})+\sum_{i=1}^{m^{+}} \operatorname{dim} H_{i}
$$

Let $c, d \in(0,+\infty)^{m^{+}} \times(-\infty, 0]^{m-m^{+}}$satisfy $\inf J_{\mathcal{Q}, B, c}>0$ and $\inf J_{\mathcal{Q}, B, d}>0$. Then for any $t \in[0,1]$,

$$
\inf J_{\mathcal{Q}, B, t c+(1-t) d}>0
$$

Proof. We use Theorem 2.9 (the infimum of $J$ can be computed on centered Gaussians) and the explicit calculations on centered Gaussian functions of Subsection 2.2. Let $Q$ be a self-adjoint linear map such that for all $x \in H, \mathcal{Q}(x)=\pi\langle x, Q x\rangle$. Let

$$
\mathcal{P}=\mathcal{P}_{\mathcal{Q}, B, m^{+}}=\left\{x \in(0,+\infty)^{m^{+}} \times(-\infty, 0]^{m-m^{+}} ; \inf J_{\mathcal{Q}, B, x}>0\right\}
$$

denote the set of exponents with prescribed signs, for which a non-trivial inequality holds. Given $c \in(0,+\infty)^{m^{+}} \times(-\infty, 0]^{m-m^{+}}$, Equation (2.3) ensures that $c \in \mathcal{P}$ if and only if

$$
\sup _{A_{k}>0} \frac{\operatorname{det}\left(\left(Q+\sum_{k} c_{k} B_{k}^{*} A_{k} B_{k}\right)_{+}\right)}{\prod_{k}\left(\operatorname{det} A_{k}\right)^{c_{k}}}<+\infty .
$$

where the supremum is on $k$-tuples of definite positive self-adjoint operators $A_{k}$ on $H_{k}$ and for a self-adjoint operator $A$ we denote

$$
(A)_{+}= \begin{cases}A & \text { if } A \text { is positive semi-definite } \\ 0 & \text { otherwise }\end{cases}
$$

When $c_{k} \neq 0$ (which is true at least for $k \leq m^{+}$), we make a change of variables $M_{k}=\left|c_{k}\right| A_{k}$ (which is still positive definite). Hence $c \in \mathcal{P}$ is equivalent to

$$
\sup _{M_{k}>0} \frac{\operatorname{det}\left(\left(Q+\sum_{i \leq m^{+}} B_{i}^{*} M_{i} B_{i}-\sum_{j>m^{+} ; c_{j} \neq 0} B_{j}^{*} M_{j} B_{j}\right)_{+}\right)}{\prod_{k}\left(\operatorname{det} M_{k}\right)^{c_{k}}}<+\infty .
$$

We claim that the latter is equivalent to

$$
\sup _{M_{k}>0} \frac{\operatorname{det}\left(\left(Q+\sum_{i \leq m^{+}} B_{i}^{*} M_{i} B_{i}-\sum_{j>m^{+}} B_{j}^{*} M_{j} B_{j}\right)_{+}\right)}{\prod_{k}\left(\operatorname{det} M_{k}\right)^{c_{k}}}<+\infty .
$$


The fact that (6.2) implies (6.3) is easy: $A \mapsto(A)_{+}$is a non-decreasing map on self-adjoint operators and the operator in the determinant of (6.3) differs from the one of (6.2) by additional negative definite terms.

To show that (6.3) implies (6.2), it is sufficient to let $M_{j}>0$ tend to 0 for all indices $j>n$ such that $c_{j}=0$ (hence $\left(\operatorname{det} M_{j}\right)^{c_{j}}=1$ ). This requires continuity properties of the numerator. Observe that $A \mapsto(A)_{+}$is not continuous (if $A$ is not positive definite but is semi-definite positive, then $(A)_{+}=A$ but for $\varepsilon>0, A-\varepsilon \mathrm{Id}$ is not positive semi-definite, so that $\left.\lim _{\varepsilon \rightarrow 0^{+}}(A-\varepsilon \mathrm{Id})_{+}=0\right)$. Fortunately, we may conclude by using the continuity of $A \mapsto \operatorname{det}\left(A_{+}\right)$(which is easy to verify: let $\left(A_{n}\right)$ be self-adjoint operators tending to $A$. If $A$ is positive definite, then so is $A_{n}$ for $n$ large enough, and continuity of the determinant allows to conclude. If there is $v \neq 0$ with $\langle A v, v\rangle<0$ then this is eventually true for $A_{n}$ and $(A)_{+}=\left(A_{n}\right)_{+}=0$. If $A$ is semi-definite positive but not definite positive, then $\operatorname{det} A=0$. If $A_{n}$ is not positive definite then $\operatorname{det}\left(A_{n}\right)_{+}=0$, while if $A_{n}$ is positive definite then $\operatorname{det}\left(A_{n}\right)_{+}=\operatorname{det} A_{n}$ tends to $\operatorname{det} A=0$ when $n$ increases).

We are ready to show the convexity of $\mathcal{P}$. Let $c, d \in \mathcal{P}$. Using that (6.3) characterizes membership to $\mathcal{P}$, we know that there exists $K_{c}$ and $K_{d}$ in $\mathbb{R}^{+}$such that for all $M_{k}>0$,

$$
\operatorname{det}\left(\left(Q+\sum_{i \leq m^{+}} B_{i}^{*} M_{i} B_{i}-\sum_{j>m^{+}} B_{j}^{*} M_{j} B_{j}\right)_{+}\right)
$$

is upper bounded by $K_{c} \prod_{k}\left(\operatorname{det} M_{k}\right)^{c_{k}}$ and by $K_{d} \prod_{k}\left(\operatorname{det} M_{k}\right)^{d_{k}}$. Therefore, for any $t \in$ $[0,1]$,

$$
\operatorname{det}\left(\left(Q+\sum_{i \leq m^{+}} B_{i}^{*} M_{i} B_{i}-\sum_{j>m^{+}} B_{j}^{*} M_{j} B_{j}\right)_{+}\right) \leq K_{c}^{t} K_{d}^{1-t} \prod_{k}\left(\operatorname{det} M_{k}\right)^{t c_{k}+(1-t) d_{k}} .
$$

The above arguments show that this implies that $t c+(1-t) d \in \mathcal{P}$. Hence $\mathcal{P}$ is convex.

\section{Positivity in The RANK ONE CASE}

We study the positivity of the optimal constant in inverse Brascamp-Lieb inequalities, when for all $k=1, \ldots, m$, $\operatorname{dim} H_{k}=1$, in terms of the coefficients $\left(c_{k}\right)_{k=1}^{m}$. In this case a very complete solution can be given, based on a rather straightforward argument. For concreteness, we may identify each $H_{k}$ with $\mathbb{R}$, and we may find non-zero vectors $u_{k} \in H$ such that $B_{k} x=\left\langle x, u_{k}\right\rangle$ for all $x \in H$. As before, we consider exponents with prescribed signs: given $m^{+} \leq m$ the first $m^{+}$coefficients are positive, while the other ones are non-positive. In addition, we work under the hypotheses of Theorem 2.9 .

For two integers $k, l$ let $\llbracket k, l \rrbracket=\{k, k+1, \ldots, l\}$ and $\rrbracket k, l \rrbracket=\{k+1, k+2, \ldots, l\}$.

7.1. No kernel. We start with the case when $\mathcal{Q}=0$. In the present setting, the hypotheses of Theorem 2.9 simply mean that the map $B_{+}$is one-to-one, that is $\left(u_{1}, \ldots, u_{m^{+}}\right)$is a basis of $H$ (which can be identified to $\mathbb{R}^{m^{+}}$). For every family $\left(f_{k}\right)_{k=1}^{m}$ of non-negative integrable functions on $\mathbb{R}$ with positive integrals, the functional of interest is

$$
J_{u, c}\left(f_{1}, \ldots, f_{m}\right)=\frac{\int_{H} \prod_{k=1}^{m} f_{k}\left(\left\langle x, u_{k}\right\rangle\right)^{c_{k}} d x}{\prod_{k=1}^{m}\left(\int_{\mathbb{R}} f_{k}\right)^{c_{k}}} .
$$

Theorem 7.1. Let $m \geq m^{+} \geq 1$ and $u_{1}, \ldots u_{m}$ be non-zero vectors in $H$. Assume also that $\left(u_{1}, \ldots, u_{m^{+}}\right)$is a basis of $H$. For $i \leq m^{+}<j$, we write that $i \sim j$ if $u_{j}$ has a non-zero $i$-th coordinate in the latter basis. Consider the positivity domain

$$
\mathcal{P}_{m^{+}}\left(\left(u_{k}\right)_{k=1}^{m}\right)=\left\{c \in(0,+\infty)^{m^{+}} \times(-\infty, 0]^{m-m^{+}} ; \inf J_{u, c}>0\right\} .
$$


For any set $S \subseteq \llbracket 1, m \rrbracket$, denote by $\mathbf{1}_{S}$ the vector in $\{0,1\}^{m}$ with $i$-th coordinate equal to 1 if and only if $i \in S$. Then

$$
\begin{aligned}
& \mathcal{P}_{m^{+}}\left(\left(u_{k}\right)_{k=1}^{m}\right)= \mathbf{1}_{\llbracket 1, m^{+} \rrbracket}+\operatorname{Pos}\left(\left\{\mathbf{1}_{\{i\}}-\mathbf{1}_{\{j\}} ; i \sim j\right\}\right) \\
&=\left\{c \in[1,+\infty)^{m^{+}} \times(-\infty, 0]^{m-m^{+}} ; \quad \sum_{k} c_{k}=m^{+}\right. \text {and } \\
&\left.\quad \text { for all } S \subset \llbracket 1, m^{+} \rrbracket, \quad \sum_{i \in S}\left(c_{i}-1\right) \leq \sum_{j ; S \sim j}\left|c_{j}\right|\right\} \\
&=\left\{c \in[1,+\infty)^{m^{+}} \times(-\infty, 0]^{m-m^{+}} ; \quad \sum_{k} c_{k}=m^{+}\right. \text {and } \\
&\left.\quad \text { for all } T \subset \rrbracket m^{+}, m \rrbracket, \quad \sum_{j \in T}\left|c_{j}\right| \leq \sum_{i ; i \sim T}\left(c_{i}-1\right)\right\},
\end{aligned}
$$

where $S \sim j$ means that there exists $i \in S$ with $i \sim j$, and $i \sim T$ means that there exists $j \in T$ with $i \sim j$, and $\operatorname{Pos}(A)$ is the positive hull of $A$.

The above description of $\mathcal{P}_{m^{+}}\left(\left(u_{k}\right)\right)$ as a positive hull can be phrased in terms of mass transportation. The following interpretation will be justified and applied in the course of the proof: consider a bipartite graph $G$ on the sets $I=\llbracket 1, m^{+} \rrbracket$ and $J=\rrbracket m^{+}, m \rrbracket$, with an edge between $i \in I$ and $j \in J$ if $i \sim j$ (i.e. $u_{j}$ has a non-zero coordinate on $u_{i}$, when decomposed in the basis $\left.\left(u_{1}, \ldots, u_{m^{+}}\right)\right)$. Then $c \in \mathcal{P}_{m^{+}}\left(\left(u_{k}\right)\right)$ if and only if one can transport the measure $\sum_{i=1}^{m^{+}}\left(c_{i}-1\right) \delta_{i}$ onto $\sum_{j=1+m^{+}}^{m}\left|c_{j}\right| \delta_{j}$ by moving the mass along the graph $G$.

Proof. For shortness, we write $\mathcal{P}$ for the positivity domain $\mathcal{P}_{m^{+}}\left(\left(u_{k}\right)_{k=1}^{m}\right)$.

First part: Let us start with $c \in \mathcal{P}$ and draw consequences of this fact. Since $\inf J_{\left(\left(u_{k}\right),\left(c_{k}\right)\right)}>0$, in particular the infimum on centered Gaussian functions is positive. Using the calculations of the previous section, this can be stated as follows: there exists $D<+\infty$ such that for all $\lambda_{1}, \ldots, \lambda_{m}>0$, it holds

$$
D \prod_{k} \lambda_{k}^{c_{k}} \geq \operatorname{det}\left(\left(\sum_{k} c_{k} \lambda_{k} u_{k} \otimes u_{k}\right)_{+}\right),
$$

where $(\cdot)_{+}$is defined as in (6.1). Recall that $(u \otimes u)(x)=\langle x, u\rangle u$. Our goal is to extract information on $c$ from (7.1). Since it is pointless when the map inside the determinant is non positive definite, we first look for values $\left(\lambda_{k}\right)$ for which the inequality has a non-trivial content.

It is convenient to work with $\left(\lambda_{k}\right)$ satisfying the following inequality

$$
\sum_{i \leq m^{+}} c_{i} \lambda_{i} u_{i} \otimes u_{i} \geq 2 \sum_{j>m^{+}}\left|c_{j}\right| \lambda_{j} u_{j} \otimes u_{j}
$$

Indeed, when the above is satisfied and since $A \geq 2 B$ implies $A-B \geq A / 2$, it follows that

$$
\sum_{k} c_{k} \lambda_{k} u_{k} \otimes u_{k} \geq \frac{1}{2} \sum_{i \leq m^{+}} c_{i} \lambda_{i} u_{i} \otimes u_{i}
$$

The map on the right-hand side is positive definite, therefore we may deduce from (7.1) that

$$
2^{m^{+}} D \prod_{k} \lambda_{k}^{c_{k}} \geq \operatorname{det}\left(\sum_{i \leq m^{+}} c_{i} \lambda_{i} u_{i} \otimes u_{i}\right)=\operatorname{det}\left(u_{1}, \ldots, u_{m^{+}}\right)^{2} \prod_{i \leq m^{+}} c_{i} \lambda_{i}
$$


Keeping in mind that (17.2) $\Longrightarrow(7.3)$, let us provide numbers $\left(\lambda_{k}\right)$ for which (17.2) is verified. For $j>m^{+}$, we denote by $\alpha_{i}(j)$ the $i$-th coordinate of $u_{j}$ in the basis $\left(u_{1}, \ldots, u_{n}\right)$. Observe that by definition $i \sim j$ means $\alpha_{i}(j) \neq 0$. Hence for all $j>m^{+}$,

$$
u_{j}=\sum_{i ; i \sim j} \alpha_{i}(j) u_{i}
$$

For any vector $v \in H$, by Cauchy-Schwarz,

$$
\left\langle v, u_{j}\right\rangle^{2} \leq\left(\sum_{i ; i \sim j} \alpha_{i}(j)^{2}\right)\left(\sum_{i ; i \sim j}\left\langle v, u_{i}\right\rangle^{2}\right) .
$$

Set $K:=\max _{j} \sum_{i ; i \sim j} \alpha_{i}(j)^{2}$. We have proved that for $j>m^{+}$,

$$
u_{j} \otimes u_{j} \leq K \sum_{i ; i \sim j} u_{i} \otimes u_{i}
$$

Summing upon $j>m^{+}$and interchanging summations in $j>m^{+}$and in $i \leq m^{+}$yield

$$
2 \sum_{j>m^{+}}\left|c_{j}\right| \lambda_{j} u_{j} \otimes u_{j} \leq 2 K \sum_{i \leq m^{+}}\left(\sum_{j ; i \sim j}\left|c_{j}\right| \lambda_{j}\right) u_{i} \otimes u_{i} .
$$

Let $q \in \mathbb{R}^{+}$and let $a \in \mathbb{R}^{m}$ satisfy

$$
i \sim j \Longrightarrow a_{i} \geq a_{j} .
$$

Set $K^{\prime}:=2 K \max _{i}\left(\sum_{j ; i \sim j}\left|c_{j}\right|\right) / c_{i}$, and

$$
\lambda_{j}:=e^{q a_{j}} \quad \text { for } \quad j>m^{+} \quad \text { and } \quad \lambda_{i}:=K^{\prime} e^{q a_{i}} \quad \text { for } \quad i \leq m^{+} .
$$

Then Inequality (7.4) readily implies that for this choice of $\lambda$, (7.2) is verified. As we have already seen, this implies that (7.3) applies and gives

$$
2^{m^{+}} D\left(\prod_{i \leq m^{+}}\left(K^{\prime}\right)^{c_{i}}\right) e^{q \sum_{k} a_{k} c_{k}} \geq \operatorname{det}\left(u_{1}, \ldots, u_{m^{+}}\right)^{2}\left(\prod_{i \leq m^{+}}\left(c_{i} K^{\prime}\right)\right) e^{q \sum_{i \leq m^{+}} a_{i}} .
$$

For $q$ tending to $+\infty$ the last inequality implies that $\sum_{k} a_{k} c_{k} \geq \sum_{i \leq m^{+}} a_{i}$. Recall that this was proved assuming (7.5) (and that $c$ is in the positivity domain of the functional $J)$. Summarizing, for $c$ in the positivity domain, and all $a \in \mathbb{R}^{m}$,

$$
\left(i \sim j \Longrightarrow a_{i} \geq a_{j}\right) \Longrightarrow \sum_{i \leq m^{+}}\left(c_{i}-1\right) a_{i} \geq \sum_{j>m^{+}}\left|c_{j}\right| a_{j} .
$$

Let us draw some consequences of this property of coefficients $c$ in the positivity domain, by making appropriate choices for $a$ :

- Choosing vectors $a$ with all equal coordinates gives $\sum_{k} c_{k}=m^{+}$, known as the homogeneity condition.

- For any $i \in \llbracket 1, m^{+} \rrbracket$, we may choose $a=\mathbf{1}_{\{i\}}$ and get $c_{i} \geq 1$.

- For $T \subset \rrbracket m^{+}, m \rrbracket$, we may define a vector $a$ as follows: $a_{j}=1$ for $j \in T, a_{j}=0$ for $j \in \rrbracket m^{+}, m \rrbracket \backslash T$, and for $i \in \llbracket 1, m^{+} \rrbracket$, set $a_{i}=1$ if $i \sim T$ and $a_{i}=0$ otherwise. It is plain that $i \sim j \Longrightarrow a_{i} \geq a_{j}$, so this vector is admissible and we can deduce that $\sum_{j \in T}\left|c_{j}\right| \leq \sum_{i ; i \sim T}\left(c_{i}-1\right)$.

- In a symmetric way, for $S \subset \llbracket 1, m^{+} \rrbracket$, we may define an admissible vector $a$ as follows: for $i \leq m^{+}, a_{i}=-1$ if $i \in S$ and $a_{i}=0$ otherwise; for $j>m^{+}$, $a_{j}=-1$ if $S \sim j$ and $a_{j}=0$ otherwise. Plugging this vector in (7.6) yields $\sum_{i \in S}\left(c_{i}-1\right) \leq \sum_{j ; S \sim j}\left|c_{j}\right|$. 
Next give a dual interpretation of (7.6) (where the right-hand side inequality is taken in the form $\left.\sum_{k} c_{k} a_{k} \geq \sum_{i \leq m^{+}} a_{i}\right)$ : for every vector $a \in \mathbb{R}^{m}$, if for all $i \leq m^{+}<j$ such that $i \sim j$, it holds $\left\langle a, \mathbf{1}_{\{i\}}-\mathbf{1}_{\{j\}}\right\rangle \geq 0$, then $\left\langle a, c-\mathbf{1}_{\llbracket 1, m^{+} \rrbracket}\right\rangle \geq 0$. Equivalently, no linear hyperplane can separate the vector $c-\mathbf{1}_{\llbracket 1, m^{+} \rrbracket}$ from the family of vectors $\left(\mathbf{1}_{\{i\}}-\mathbf{1}_{\{j\}}\right)_{i \sim j}$. By the Hahn-Banach theorem, this implies that $c-\mathbf{1}_{\llbracket 1, m^{+} \rrbracket}$ belongs to the convex cone generated by the vectors $\left(\mathbf{1}_{\{i\}}-\mathbf{1}_{\{j\}}\right)_{i \sim j}$. This concludes the first part of the proof.

Second part: let us show that $\mathbf{1}_{\llbracket 1, m^{+} \rrbracket}+\operatorname{Pos}\left(\left(\mathbf{1}_{\{i\}}-\mathbf{1}_{\{j\}}\right)_{i \sim j}\right)$ is included in the positivity domain $\mathcal{P}$ of the functional $J$. Since Proposition 6.1 ensures that $\mathcal{P}$ is convex, it is enough to show that for all $i \leq m^{+}<j$ such that $i \sim j$, it contains the half-line $\mathbf{1}_{\llbracket 1, m^{+} \rrbracket}+\mathbb{R}^{+}\left(\mathbf{1}_{\{i\}}-\mathbf{1}_{\{j\}}\right)$.

Let us start with observing that $\mathbf{1}_{\llbracket 1, m^{+} \rrbracket} \in \mathcal{P}$. Indeed, for all measurable $f_{k}: \mathbb{R} \rightarrow \mathbb{R}^{+}$, by the change of variables $y_{i}:=\left\langle x, u_{i}\right\rangle, i \in \llbracket 1, m^{+} \rrbracket$ and Fubini's theorem

$$
\int_{H} \prod_{i=1}^{m^{+}} f_{i}\left(\left\langle x, u_{i}\right\rangle\right) d x=\left|\operatorname{det}\left(\left(u_{i}\right)_{i=1}^{m^{+}}\right)\right|^{-1} \int_{\mathbb{R}^{m^{+}}} \prod_{i=1}^{m^{+}} f_{i}\left(y_{i}\right) d y=\left|\operatorname{det}\left(\left(u_{i}\right)_{i=1}^{m^{+}}\right)\right|^{-1} \prod_{i=1}^{m^{+}} \int_{\mathbb{R}} f_{i} .
$$

Another basic ingredient, which is actually the simplest instance of the reverse inequalities we are investigating, is the reverse Hölder inequality: for $\varepsilon \geq 0$ and $f, g$ non-negative measurable functions on $\mathbb{R}$,

$$
\int f^{1+\varepsilon} g^{-\varepsilon} \geq\left(\int f\right)^{1+\varepsilon}\left(\int g\right)^{-\varepsilon}
$$

We are ready to show that for $i_{0} \sim j, \mathbf{1}_{\llbracket 1, m^{+} \rrbracket}+\mathbb{R}^{+}\left(\mathbf{1}_{\left\{i_{0}\right\}}-\mathbf{1}_{\{j\}}\right) \subset \mathcal{P}$. Let $\varepsilon \geq 0$. Then, using that $u_{j}=\sum_{i} \alpha_{i}(j) u_{i}$ with $\alpha_{i_{0}}(j) \neq 0$, changing variables by $y_{i}:=\left\langle x, u_{i}\right\rangle, i \in \llbracket 1, m^{+} \rrbracket$ as above

$$
\begin{aligned}
& \int_{H}\left(\prod_{i \in \llbracket 1, m^{+} \rrbracket \backslash\left\{i_{0}\right\}} f_{i}\left(\left\langle x, u_{i}\right\rangle\right)\right) f_{i_{0}}\left(\left\langle x, u_{i_{0}}\right\rangle\right)^{1+\varepsilon} f_{j}\left(\left\langle x, u_{j}\right\rangle\right)^{-\varepsilon} d x \\
= & \int_{H}\left(\prod_{i \in \llbracket 1, m^{+} \rrbracket \backslash\left\{i_{0}\right\}} f_{i}\left(\left\langle x, u_{i}\right\rangle\right)\right) f_{i_{0}}\left(\left\langle x, u_{i_{0}}\right\rangle\right)^{1+\varepsilon} f_{j}\left(\sum_{i \leq m^{+}} \alpha_{i}(j)\left\langle x, u_{i}\right\rangle\right)^{-\varepsilon} d x \\
= & \left|\operatorname{det}\left(\left(u_{i}\right)_{i=1}^{m^{+}}\right)\right|^{-1} \int_{\mathbb{R}^{m^{+}}}\left(\prod_{i \in \llbracket 1, m^{+} \rrbracket \backslash\left\{i_{0}\right\}} f_{i}\left(y_{i}\right)\right) f_{i_{0}}\left(y_{i_{0}}\right)^{1+\varepsilon} f_{j}\left(\sum_{i \leq m^{+}} \alpha_{i}(j) y_{i}\right)^{-\varepsilon} d y .
\end{aligned}
$$

Applying inverse Hölder in the variable $y_{i_{0}}$ and using $\alpha_{i_{0}}(j) \neq 0$, we deduce that for any $\left(y_{i}\right)_{i \in \llbracket 1, m^{+} \rrbracket \backslash\left\{i_{0}\right\}}$

$$
\int_{\mathbb{R}} f_{i_{0}}\left(y_{i_{0}}\right)^{1+\varepsilon} f_{j}\left(\sum_{i \leq m^{+}} \alpha_{i}(j) y_{i}\right)^{-\varepsilon} d y_{i_{0}} \geq\left(\int f_{i_{0}}\right)^{1+\varepsilon}\left(\frac{1}{\alpha_{i_{0}}(j)} \int f_{j}\right)^{-\varepsilon}
$$

Plugging this estimate in the latter integral over $\mathbb{R}^{n}$ we arrive at

$$
\begin{aligned}
& \int_{H}\left(\prod_{i \in \llbracket 1, m^{+} \rrbracket \backslash\left\{i_{0}\right\}} f_{i}\left(\left\langle x, u_{i}\right\rangle\right)\right) f_{i_{0}}\left(\left\langle x, u_{i_{0}}\right\rangle\right)^{1+\varepsilon} f_{j}\left(\left\langle x, u_{j}\right\rangle\right)^{-\varepsilon} d x \\
\geq & \left|\operatorname{det}\left(\left(u_{i}\right)_{i=1}^{m^{+}}\right)\right|^{-1} \alpha_{i_{0}}(j)^{-\varepsilon} \prod_{\left.i \in \llbracket 1, m^{+} \rrbracket \backslash i_{0}\right\}}\left(\int f_{i}\right) \times\left(\int f_{i_{0}}\right)^{1+\varepsilon}\left(\int f_{j}\right)^{-\varepsilon} .
\end{aligned}
$$

This inequality proves that $\mathbf{1}_{\llbracket 1, m^{+} \rrbracket}+\varepsilon\left(\mathbf{1}_{\left\{i_{0}\right\}}-\mathbf{1}_{\{j\}}\right) \in \mathcal{P}$.

Third part: Our final task is to show that the descriptions of $\mathcal{P}$ is terms of inequalities coincides with the one in terms of positive hull. This can be done "by hands," but we 
present a neat argument in terms of transportation plans. We have shown that $c \in \mathcal{P}$ is equivalent to $c-\mathbf{1}_{\llbracket 1, m^{+} \rrbracket} \in \operatorname{Pos}\left(\left(\mathbf{1}_{\{i\}}-\mathbf{1}_{\{j\}}\right)_{i \sim j}\right)$. The latter is equivalent to the existence of non-negative coefficients $\left(\gamma_{i, j}\right)_{i \sim j}$ such that $c-\mathbf{1}_{\llbracket 1, m^{+} \rrbracket}=\sum_{i \sim j} \gamma_{i, j}\left(\mathbf{1}_{\{i\}}-\mathbf{1}_{\{j\}}\right)$, or in coordinates:

$$
\begin{aligned}
& \text { for } i \leq m^{+}, \quad c_{i}-1=\sum_{j ; i \sim j} \gamma_{i, j}, \\
& \text { for } j>m^{+}, \quad\left|c_{j}\right|=\sum_{i ; i \sim j} \gamma_{i, j} .
\end{aligned}
$$

This can be interpreted as a coupling, or transportation plan between measures: $\gamma_{i, j}$ represents the amount of mass which is transported from $i$ to $j$, and such a shipping is allowed only if $i \sim j$. Therefore $c$ belongs to $\mathcal{P}$ if and only if it is possible to transport the measure $\sum_{i \leq m^{+}}\left(c_{i}-1\right) \delta_{i}$ to the measure $\sum_{j>m^{+}}\left|c_{j}\right| \delta_{j}$ (or vice-versa), while carrying mass only between points which are in relation for $\sim$. This questions of existence of transport with constraints is well know. Its solution is given in the following classical lemma and it allows to complete the proof. Observe that the indices $i \leq m^{+}$and $j>m^{+}$play symmetric role for the transportation problem, which leads to two different description of $\mathcal{P}$ in terms of inequalities.

Lemma 7.2. Let $I$ and $J$ be disjoint finite sets. Let $E \subset I \times J$ and consider the bipartite graph $(I, J ; E)$. Let $\left(\alpha_{i}\right)_{i \in I}$ and $\left(\beta_{j}\right)_{j \in J}$ be non-negative numbers. Then there exists a transportation plan along the graph between $\sum_{i \in I} \alpha_{i} \delta_{i}$ and $\sum_{j \in J} \beta_{i} \delta_{i}$ if and only if:

$$
\sum_{i \in I} \alpha_{i}=\sum_{j \in J} \beta_{j} \text { and for all } S \subset I ; \sum_{i \in S} \alpha_{i} \leq \sum_{j ; S \sim j} \beta_{j}
$$

Proof. The condition means that the origin and target measures have same total mass, and that the mass of any subset of the origin set is not larger than the mass for the target measure of the set of its neighbors.

Showing that the existence of a transport plan implies the above inequalities is straightforward, and actually not the direction we need for the previous theorem, so we omit it.

Assume that (7.7) is verified. Let us build a weighted graph $G$ by enriching $(I, J ; E)$ as follows: we assign to every existing edge $(i \sim j)$ a weight $w:=1+\sum_{i \in I} \alpha_{i}$; we also add a vertex $A$ and connect it to each $i \in I$ with a weight $\alpha_{i}$ on the edge; eventually we add another vertex $B$ and connect to each $j \in J$ with a weight $\beta_{i}$.

Our goal is to show that the maximal flow between $A$ and $B$ is equal to $\sum_{i \in I} \alpha_{i}$ (which means that all the mass from $I$ can be transported to $B$ along the graph, and since $\sum_{i} \alpha_{i}=\sum_{j} \beta_{j}$ all the target mass is reached). By the Max flow-Min cut theorem (see e.g. [40]), it is enough to show that the minimal weight of a cut separating $A$ and $B$ is equal to $\sum_{i \in I} \alpha_{i}$ (with corresponds to cutting all the edges incident to $A$ ).

Let us study a minimal cut. First, since the edges between $I$ and $J$ have weight $w>\sum_{i \in I} \alpha_{i}$, they are not in a minimal cut. Such a cut is thus as follows: there are subsets $S \subset I$ and $T \subset J$ such that $S^{c}=I \backslash S$ and $T^{c}=J \backslash T$ are not connected, and one cuts the edges between $A$ and $I$ and the ones between $B$ and $J$. The weight of this cut is

$$
\sum_{i \in S} \alpha_{i}+\sum_{j \in T} \beta_{j}
$$

Our goal is to bound this weight from below as follows,

$$
\sum_{i \in S} \alpha_{i}+\sum_{j \in T} \beta_{j} \geq \sum_{i \in I} \alpha_{i}
$$


This is equivalent, after canceling the terms appearing twice, to $\sum_{i \in S^{c}} \alpha_{i} \leq \sum_{j \in T} \beta_{j}$. This is indeed true: by hypothesis $\sum_{i \in S^{c}} \alpha_{i} \leq \sum_{j ; S^{c} \sim j} \beta_{j}$. But since $S^{c}$ and $T^{c}$ are not connected, $S^{c} \sim j$ implies that $j \in T$, and the latter sum is at most $\sum_{j \in T} \beta_{j}$, as claimed.

7.2. With a kernel. Here we consider in addition a kernel $e^{-\mathcal{Q}}$, with the restriction that $s^{+}(\mathcal{Q}), s^{-}(\mathcal{Q}) \leq 1$. As above we work under the assumptions $\mathcal{Q}_{\mid \text {ker } B_{+}}$positive definite and $\operatorname{dim} H \geq s^{+}(\mathcal{Q})+\sum_{i=1}^{m^{+}} \operatorname{dim} H_{i}$, for which a convenient equivalent form is given in Lemma 3.1. In our setting, they can be rephrased as follows (we introduce a small twist with respect to the decomposition in the lemma, namely a dilation which allows for a more concrete decomposition): there are vectors $u_{0}, u_{m+1} \in H$ such that for all $x \in H$,

$$
\mathcal{Q}(x)=\pi\left\langle x, u_{0}\right\rangle^{2}-\pi\left\langle x, u_{m+1}\right\rangle^{2} .
$$

Note that these two vectors may be equal to zero (e.g. if $Q$ is non-positive, $u_{0}=0$ ). Moreover setting $B_{0} x=\left\langle x, u_{0}\right\rangle$ and $B_{m+1} x=\left\langle x, u_{m+1}\right\rangle$, we know that ker $B_{+} \subset \operatorname{ker} B_{m+1}$ and that $B_{0+}: H \rightarrow B_{0} H \times B_{1} H \times \cdots \times B_{m^{+}} H$ is one to one. The former is equivalent to $\bigcap_{i=1}^{m^{+}} u_{i}^{\perp} \subset u_{m+1}^{\perp}$, that is

$$
u_{m+1} \in \operatorname{vect}\left\{u_{1}, \ldots, u_{m^{+}}\right\}
$$

while the latter means that:

- either $u_{0}=0$ and $\left(u_{1}, \ldots, u_{m^{+}}\right)$is a basis of $H$,

- or $\left(u_{0}, u_{1}, \ldots, u_{m^{+}}\right)$is a basis of $H$.

In any of the above cases, we denote by $\mathbb{U}$ the corresponding basis of $H$. Given $i \in I=$ $\llbracket 0, m^{+} \rrbracket$ and $j \in J=\rrbracket m^{+}, m+1 \rrbracket$, we write $i \sim j$ if $u_{j}$, once decomposed in the basis $\mathbb{U}$, has a positive coordinate on the vector $u_{i}$ of the basis. This relation creates a bipartite graph $G$ on $I$ and $J$. Note that $m+1$ is an isolated vertex of the graph when $u_{m+1}=0$, and so is 0 when $u_{0}=0$. The functional of interest is

$$
J_{\mathcal{Q},\left(u_{k}\right)_{k=1}^{m}, c}\left(f_{1}, \ldots, f_{m}\right)=\frac{\int_{H} e^{-\pi\left\langle x, u_{0}\right\rangle^{2}+\pi\left\langle x, u_{m+1}\right\rangle^{2}} \prod_{k=1}^{m} f_{k}\left(\left\langle x, u_{k}\right\rangle\right)^{c_{k}} d x}{\prod_{k=1}^{m}\left(\int_{\mathbb{R}} f_{k}\right)^{c_{k}}} .
$$

Now comes a description of its positivity domain

$$
\mathcal{P}_{m^{+}}\left(\mathcal{Q},\left(u_{k}\right)_{k=1}^{m}\right)=\left\{c \in(0,+\infty)^{m^{+}} \times(-\infty, 0]^{m-m^{+}} ; \text {inf } J_{\mathcal{Q},\left(u_{k}\right)_{k=1}^{m}, c}>0\right\} .
$$

Theorem 7.3. With the above notation and hypotheses,

$$
\begin{aligned}
& \mathcal{P}_{m^{+}}\left(\mathcal{Q},\left(u_{k}\right)_{k=1}^{m}\right)= \mathbf{1}_{\llbracket 1, m^{+} \rrbracket}+\operatorname{Pos}\left(\left\{\mathbf{1}_{\{i\}} ; i \sim m+1\right\} \cup\left\{-\mathbf{1}_{\{j\}} ; 0 \sim j\right\}\right. \\
&\left.\cup\left\{\mathbf{1}_{\{i\}}-\mathbf{1}_{\{j\}} ; 1 \leq i \sim j \leq m\right\}\right)\left\{c \in[1,+\infty)^{m^{+}} \times(-\infty, 0]^{m-m^{+}} ;\right. \\
& \quad \text { for all } S \subset \llbracket 1, m^{+} \rrbracket \text { with } S \nsim m+1, \quad \sum_{i \in S}\left(c_{i}-1\right) \leq \sum_{j ; S \sim j}\left|c_{j}\right|, \\
&\left.\quad \text { and for all } T \subset \rrbracket m^{+}, m \rrbracket \text { with } 0 \nsim T, \quad \sum_{j \in T}\left|c_{j}\right| \leq \sum_{i ; i \sim T}\left(c_{i}-1\right)\right\}, \\
&=\operatorname{Proj}_{\mathbb{R}^{\llbracket 1, m \rrbracket}}\left(\mathcal{P}_{1+m^{+}}\left(u_{0}, u_{1}, \ldots, u_{m}, u_{m+1}\right)\right) .
\end{aligned}
$$

Let us comment on this statement before proving it. The notation of the last line, involving a projection and an extended use of the notation of the positivity domain in the case of no kernel (if $u_{0}$ or $u_{m+1}$ is zero, just discard it), means the following: inf $J_{\mathcal{Q},\left(u_{k}\right)_{k=1}^{m}, c}>0$ 
if and only if there exists $c_{0} \geq 1$ and $c_{m+1} \leq 0$ and $\varepsilon>0$ such that for all $f_{k}: \mathbb{R} \rightarrow \mathbb{R}^{+}$ $(k=0,1, \ldots, m+1)$ integrable and with positive integral:

$$
\int_{H} f_{0}\left(\left\langle x, u_{0}\right\rangle\right)^{c_{0}} f_{m+1}\left(\left\langle x, u_{m+1}\right\rangle\right)^{c_{m+1}} \prod_{k=1}^{m} f_{k}\left(\left\langle x, u_{k}\right\rangle\right)^{c_{k}} d x \geq \varepsilon \prod_{k=0}^{m+1}\left(\int_{H_{k}} f_{k}\right)^{c_{k}} .
$$

Here $H_{k}$ is the image of $H$ by $x \mapsto\left\langle x, u_{k}\right\rangle$. If for instance $u_{0}=0$ then $H_{0}=\{0\}$ and the term $f_{0}(0)=\int_{H_{0}} f_{0}>0$ appears on both sides, and can be discarded. In other words, the positivity of the constant in the inequality with kernel can be deduced from an inequality without kernel, by specifying one or two functions to be Gaussian.

The description of the positivity domain as a positive convex hull can also be interpreted in terms of a transportation problem: $c$ is in the positivity domain if and only if one can transport the measure $\sum_{i=1}^{m^{+}}\left(c_{i}-1\right) \delta_{i}$ to $\sum_{j=1+m^{+}}^{m}\left|c_{j}\right| \delta_{j}$ along the bipartite graph $G$ defined above, with the help of a source at 0 and of a sink at $m+1$.

Proof of Theorem 7.3. The strategy is the same as for Theorem 7.1, so we only explain the changes. We simply write $\mathcal{P}$ for the positivity domain. Let us denote by $\mathcal{P}_{1}, \mathcal{P}_{2}$ and $\mathcal{P}_{3}$ the three sets appearing in the claim (in the same order).

Let $c \in \mathcal{P}$. By Theorem 2.9 and Gaussian calculations, there exists $D>0$ such that for all $\lambda_{1}, \ldots, \lambda_{m}$,

$$
D \prod_{k} \lambda_{k}^{c_{k}} \geq \operatorname{det}\left(\left(\sum_{k=0}^{m+1} c_{k} \lambda_{k} u_{k} \otimes u_{k}\right)_{+}\right),
$$

where we have set $c_{0}=1, c_{m+1}=-1, \lambda_{0}=\lambda_{m+1}=1$ in order to include the terms coming from the kernel. Our first task is to infer that for every $a \in \mathbb{R}^{\llbracket 0, m+1 \rrbracket}$ satisfying $a_{0}=a_{m+1}=0$,

$$
\left(i \sim j \Longrightarrow a_{i} \geq a_{j}\right) \Longrightarrow \sum_{i \leq m^{+}}\left(c_{i}-1\right) a_{i} \geq \sum_{j>m^{+}}\left|c_{j}\right| a_{j}
$$

To do this we look for numbers $b_{k}$ such that for all $q \geq 0$, Inequality (7.2) is verified for $\lambda_{k}=\lambda_{k}(q)=b_{k} e^{q a_{k}}$. Letting $q$ tend to infinity in the determinant inequality then yields (7.10). The main changes in the argument come from the "boundary" conditions $\lambda_{0}(q)=\lambda_{m+1}(q)=1$ which force $a_{0}=a_{m+1}=0$ and $b_{0}=b_{m+1}=1$. The strategy is again to choose the $\lambda_{k}$ such that (17.4) holds. Observe that (17.4) is verified when for all $i \leq m^{+}, 2 K \sum_{j ; i \sim j}\left|c_{j}\right| \lambda_{j} \leq c_{i} \lambda_{i}$. Setting $M:=\max (1,2 K(m+1))$, we get that a sufficient condition to ensure the latter is to have:

$$
i \sim j \Longrightarrow M\left|c_{j}\right| \lambda_{j} \leq c_{i} \lambda_{i} .
$$

As already mentioned, we look for $\lambda_{k}=b_{k} e^{q a_{k}}$ and $a$ verifies $i \sim j \Longrightarrow a_{i} \geq a_{j}$. Hence it is enough to choose $b$ such that

$$
i \sim j \Longrightarrow M\left|c_{j}\right| b_{j} \leq c_{i} b_{i}
$$

Recall that $c_{0} b_{0}=\left|c_{m+1}\right| b_{m+1}=1$ so the latter inequality may fail, but thanks to (7.8) $0 \nsim m+1$. Eventually, if we choose $\left(b_{k}\right)$ such that $b_{i}=M / c_{i}$ for $i \in \llbracket 1, m^{+} \rrbracket$ and $\left|c_{j}\right| b_{j} \leq 1 / M$ for $j \in \rrbracket m^{+}, m \rrbracket$, then (7.11) is verified. Thus $c \in \mathcal{P}$ implies (17.10). Using the Hahn-Banach Theorem, (7.10) means $c \in \mathcal{P}_{1}$. So we have proved that $\mathcal{P} \subset \mathcal{P}_{1}$.

Next we show that $\mathcal{P}_{1} \subset \mathcal{P}_{2}$ by drawing consequences of (17.10);

- For any $i \in \llbracket 1, m^{+} \rrbracket$, we may choose $a=\mathbf{1}_{\{i\}}$ and get $c_{i} \geq 1$.

- For $T \subset \rrbracket m^{+}, m \rrbracket$ with $0 \nsim T$ we may define a vector $a$ as follows: $a_{j}=1$ for $j \in T$, $a_{i}=1$ if $i \sim T$ and $a_{k}=0$ otherwise. It readily verifies the hypothesis of (17.10), so we can deduce that $\sum_{j \in T}\left|c_{j}\right| \leq \sum_{i ; i \sim T}\left(c_{i}-1\right)$. 
- In a symmetric way, for $S \subset \llbracket 1, m^{+} \rrbracket$ with $S \nsim m+1$ we may define an admissible vector $a$ as follows: $a_{i}=-1$ is $i \in S ; a_{j}=-1$ if $S \sim j$ and $a_{k}=0$ otherwise. This implies $\sum_{i \in S}\left(c_{i}-1\right) \leq \sum_{j ; S \sim j}\left|c_{j}\right|$.

Now we prove that $\mathcal{P}_{2} \subset \mathcal{P}_{3}$. Let $c \in \mathcal{P}_{2}$, and set $\alpha_{i}=c_{i}-1$ for $1 \leq i \leq m^{+}$and $\beta_{j}=\left|c_{j}\right|$ for $m^{+}<j \leq m$. Let us consider the bipartite graph $\tilde{G}$ on $I=\llbracket 0, m^{+} \rrbracket$ and $J=\rrbracket m^{+}, m+1 \rrbracket$ obtained by adding to $G$ an edge between 0 and $m+1$. Let us choose two numbers $\alpha_{0}$ and $\beta_{m+1}$ such that $\sum_{i \in I} \alpha_{i}=\sum_{j \in J} \beta_{j}$ and $\alpha_{0}, \beta_{m+1}>\sum_{i \in \llbracket 1, m^{+} \rrbracket} \alpha_{i}+\sum_{j \in \rrbracket m^{+}, m \rrbracket} \beta_{j}$.

Let us show that it is possible to transport along $\tilde{G}$ the measure $\sum_{i \in I} \alpha_{i} \delta_{i}$ to $\sum_{j \in J} \beta_{j} \delta_{j}$, by application of Lemma 7.2. The equality of masses holds by construction. It remains to prove that for every $S \subset I, \sum_{i \in S} \alpha_{i} \leq \sum_{j \in N(S)} \beta_{j}$, where $N(S)$ denotes the set of vertices which are connected to $S$ in $\tilde{G}$. Let us consider several cases

- If $0 \notin S$ and $S \nsim m+1$ then the inequality comes from the hypothesis that $c \in \mathcal{P}_{2}$.

- If $0 \notin S$ and $S \sim m+1$, the inequality holds simply because the term $\beta_{m+1}$ is larger than $\sum_{i=1}^{m^{+}} \alpha_{i} \geq \sum_{i \in S} \alpha_{i}$.

- If $0 \in S$, then by construction $m+1 \in N(S)$. Our aim is to show that $\sum_{i \in S} \alpha_{i} \leq$ $\sum_{j \in N(S)} \beta_{j}$. Subtracting from the equality of masses condition shows that the inequality is equivalent to $\sum_{i \in I \backslash S} \alpha_{i} \geq \sum_{j \in J \backslash N(S)} \beta_{j}$. Define $T:=J \backslash N(S)$. Observe that $T \subset \rrbracket m^{+}, m \rrbracket$ since $m+1 \in N(S)$. Moreover by construction $N(T) \subset$ $I \backslash S$ does not contain 0 as $S$ does. So the fact that $c \in \mathcal{P}_{2}$ ensures that $\sum_{j \in T} \beta_{j} \leq$ $\sum_{i \in N(T)} \alpha_{i}$. Since $N(T) \subset I \backslash S$ we obtain $\sum_{i \in I \backslash S} \alpha_{i} \geq \sum_{j \in J \backslash N(S)} \beta_{j}$ as needed.

By Lemma 7.2 there is a transport along $\tilde{G}$. It may ship an amount $\gamma$ of mass between the vertices 0 and $m+1$. If we remove this amount from the initial mass at these two points, we get two distributions which admit a transport which does not use the edge between 0 and $m+1$. In other words if we set $c_{0}=1+\alpha_{0}-\gamma \geq 1$ and $c_{m+1}=-\left(\beta_{m+1}-\gamma\right) \leq$ 0 , we have shown that there is a transportation plan along $G$ from $\sum_{i=0}^{m^{+}}\left(c_{i}-1\right) \delta_{i}$ to $\sum_{j=1+m^{+}}^{m+1}\left|c_{j}\right| \delta_{j}$. According to Theorem 7.1 and its interpretation in terms of transport, this means that $\left(c_{0}, c_{1}, \ldots, c_{m+1}\right)$ is in the positivity domain $\mathcal{P}_{1+m^{+}}\left(u_{0}, u_{1}, \ldots, u_{m+1}\right)$ of an inverse Brascamp-Lieb inequality without kernel (but with more functions).

So starting from $c=\left(c_{1}, \ldots, c_{m}\right) \in \mathcal{P}_{2}$ we have expressed it as the projection of a vector $\left(c_{0}, c_{1}, \ldots, c_{m+1}\right)$ in $\mathcal{P}_{1+m^{+}}\left(u_{0}, u_{1}, \ldots, u_{m+1}\right)$. This concludes the proof of $\mathcal{P}_{2} \subset \mathcal{P}_{3}$. The particular cases when $u_{0}$ (or $u_{m+1}$ ) is zero is also treated by this argument because in this case 0 is isolated in $G$ and thus it is not involved in the transport.

The inclusion $\mathcal{P}_{3} \subset \mathcal{P}$ is immediate. It $c \in \mathcal{P}_{3}$, then by Theorem 7.1 there exists $c_{0}>0$, $c_{m+1} \leq 0$ and $\varepsilon>0$ such that for all non-negative integrable functions $f_{k}, k=0, \ldots, m+1$,

$$
\int_{H} f_{0}\left(\left\langle x, u_{0}\right\rangle\right)^{c_{0}} f_{m+1}\left(\left\langle x, u_{m+1}\right\rangle\right)^{c_{m+1}} \prod_{k=1}^{m} f_{k}\left(\left\langle x, u_{k}\right\rangle\right)^{c_{k}} d x \geq \varepsilon \prod_{k=0}^{m+1}\left(\int_{H_{k}} f_{k}\right)^{c_{k}} .
$$

It remains to choose adequate Gaussian functions $f_{0}$ and $f_{m+1}$ so that

$$
f_{0}\left(\left\langle x, u_{0}\right\rangle\right)^{c_{0}} f_{m+1}\left(\left\langle x, u_{m+1}\right\rangle\right)^{c_{m+1}} \leq e^{-\pi\left\langle x, u_{0}\right\rangle^{2}+\pi\left\langle x, u_{m+1}\right\rangle^{2}}=e^{-\mathcal{Q}(x)}
$$

to get a non-trivial inequality for the initial functional. It is possible to achieve equality when $c_{m+1}<0$; we use an inequality in case $c_{m+1}=0$.

\section{Positivity CONDition in the General CASE}

We turn to a positivity condition in the general case. Let $0 \leq m^{+} \leq m$ and for $k=0, \ldots, m+1$, let $B_{k}: H \rightarrow H_{k}$ be a surjective linear map. Recall that $B_{+}$denotes the map $\left(B_{1}, \ldots, B_{m^{+}}\right): H \rightarrow H_{1} \times \cdots \times H_{m^{+}}$. With this notation, $\operatorname{ker} B_{+}=\bigcap_{k=1}^{m^{+}} \operatorname{ker} B_{k}$. 
Similarly we define $B_{0+}=\left(B_{0}, B_{1}, \ldots B_{m^{+}}\right): H \rightarrow H_{0} \times \cdots \times H_{m^{+}}$. Recall also the non-degeneracy conditions (2.4) and (2.5), which we assume from now on.

8.1. Recursive structure of the problem. Any linear subspace $V \subseteq H$, together with the quotient space $H / V$, yields a split of $H$, i.e. the following sequence is exact

$$
0 \longrightarrow \mathrm{O} \stackrel{i}{\longrightarrow} H \stackrel{\pi}{\longrightarrow} H / V \longrightarrow 0,
$$

where $i: V \rightarrow H$ is the natural embedding and $\pi: H \rightarrow H / V$ is the natural quotient map.

Next, for each $k=0, \ldots, m+1$ denote

$$
V_{k}=B_{k} V \text {. }
$$

We consider a split of $H_{k}$ induced from the split of $H$ by the map $B_{k}$, namely

$$
0 \longrightarrow V_{k} \stackrel{i_{k}}{\longrightarrow} H_{k} \stackrel{\pi_{k}}{\longrightarrow} H_{k} / V_{k} \longrightarrow 0,
$$

together with surjective linear maps $b_{k}: V \rightarrow V_{k}$ and $\beta_{k}:{ }^{H} / V \rightarrow{ }_{k}{ }_{k} / V_{k}$ defined such that the diagram

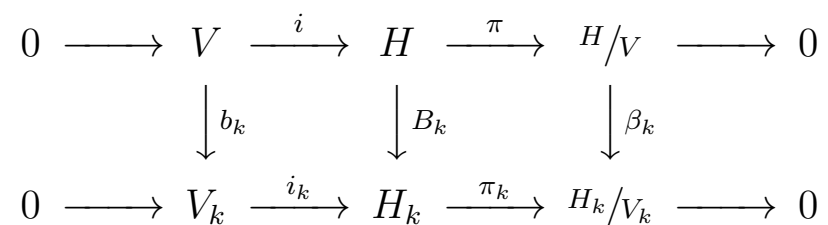

commutes. In other words, $b_{k}$ is the restriction of $B_{k}$ to $V$, while $\beta_{k}$ is the quotient of $B_{k}$ by $V$, which can be defined explicitly by

$$
\beta_{k}(x+V)=B_{k} x+V_{k} .
$$

Similarly as for the maps $B_{k}$ we consider the maps

$$
\begin{aligned}
b_{+} & =\left(b_{1}, \ldots, b_{m^{+}}\right): V \rightarrow V_{1} \times \cdots \times V_{m^{+}}, \\
b_{0+} & =\left(b_{0}, b_{1}, \ldots, b_{m^{+}}\right): V \rightarrow V_{0} \times V_{1} \times \cdots \times V_{m^{+}}, \\
\beta_{+} & =\left(\beta_{1}, \ldots, \beta_{m^{+}}\right): H / V \rightarrow H_{1} / V_{1} \times \cdots \times H_{m^{+}} / V_{m^{+}}, \\
\beta_{0+} & =\left(\beta_{0}, \beta_{1}, \ldots, \beta_{m^{+}}\right): H / V \rightarrow H_{0} / V_{0} \times H_{1} / V_{1} \times \cdots \times H_{m^{+}} / V_{m^{+}} .
\end{aligned}
$$

In the sequel, the above construction of restriction and quotient of maps will be applied recursively to $V$ and the maps $b_{k}: V \rightarrow V_{k}$ as well as to $H / V$ and the maps $\beta_{k}:{ }^{H} / V \rightarrow H_{k} / V_{k}$.

Note a simple fact concerning the kernel of a quotient map.

Lemma 8.1. Let $B: H \rightarrow H^{\prime}$ be a linear map (not necessarily surjective), $V \subseteq H$ be a subspace and denote $V^{\prime}=B V$. Consider the map $\beta:{ }^{H} / V \rightarrow H^{\prime} / V^{\prime}$ defined as a quotient of $B$, i.e. $\beta(x+V)=B x+V^{\prime}$. Then its kernel verifies

$$
\pi^{-1}(\operatorname{ker} \beta)=V+\operatorname{ker} B,
$$

where $\pi: H \rightarrow H / V$ is the canonical projection.

Proof. The inclusions $V \subseteq \pi^{-1}(\operatorname{ker} \beta)$ and $\operatorname{ker} B \subseteq \pi^{-1}(\operatorname{ker} \beta)$ are obvious. For the other inclusion, if $x \in \pi^{-1}(\operatorname{ker} \beta)$, i.e. $B x \in V^{\prime}=B V$, then there exists $v \in V$ such that $B x=B v$ and hence $x=v+(x-v) \in V+\operatorname{ker} B$.

The following notion will be crucial:

Definition 8.2. Given the maps $B_{k}: H \rightarrow H_{k}$ for $k=0,1, \ldots, m^{+}$and a linear subspace $V \subset H$, we call the split $0 \longrightarrow V \longrightarrow H \longrightarrow H / V \longrightarrow 0$ admissible for $(H, B)$ if the map $b_{0+}$ is a linear isomorphism. For shortness we also say that $V$ is an admissible subspace, and omit to mention $(H, B)$ when there is no ambiguity. 
The next lemma tells that Condition (3.1) is inherited by subspaces and quotients induced by admissible splits.

Lemma 8.3. Suppose here that the map $B_{0+}$ is a linear isomorphism and consider a split $0 \longrightarrow \mathrm{V} \longrightarrow \mathrm{H} \longrightarrow \mathrm{H} / \mathrm{V} \longrightarrow 0$. Then the map $b_{0+}$ is injective and the map $\beta_{0+}$ is surjective. Moreover, the following assertions are equivalent:

(i) the split is admissible (i.e. the map $b_{0+}$ is a linear isomorphism),

(i') the map $b_{0+}$ is surjective,

(ii) $\operatorname{dim} V=\sum_{i=0}^{m^{+}} \operatorname{dim} B_{i} V$,

(iii) the map $\beta_{0+}$ is a linear isomorphism,

(iii') the map $\beta_{0+}$ is injective,

(iv) $\bigcap_{i=0}^{m^{+}}\left(V+\operatorname{ker} B_{i}\right)=V$.

Proof. Since $B_{0+}: H \rightarrow H_{0} \times \cdots \times H_{m^{+}}$is a linear isomorphism, it is clear that its restriction $b_{0+}: V \rightarrow V_{0} \times \cdots \times V_{m^{+}}$is injective (recall the notation $V_{k}=B_{k} V$ ). Hence (i) $\Longleftrightarrow$ (i'). The fact that $B_{0+}$ is onto also ensures that its quotient $\beta_{0+}:{ }^{H} / V \rightarrow$ $H_{0} / V_{0} \times \cdots \times H_{m^{+}} / V_{m^{+}}$is surjective. Hence (iii) $\Longleftrightarrow$ (iii').

Next we use the basic fact that a linear map between finite dimensional vector spaces $L: X \rightarrow Y$ is bijective if and only if $\operatorname{dim} X=\operatorname{dim} Y$ and $L$ is injective (which is also equivalent to $\operatorname{dim} X=\operatorname{dim} Y$ and $L$ is surjective). This directly yields (i) $\Longleftrightarrow$ (ii).

Since $B_{0+}$ is an isomorphism, it holds $\operatorname{dim} H=\sum_{i=0}^{m^{+}} \operatorname{dim} H_{i}$. Therefore, by subtraction, (ii) is equivalent to

$$
\operatorname{dim} H / V=\sum_{i=0}^{m^{+}} \operatorname{dim} H_{i} / V_{i} .
$$

Since $\beta_{0+}$ is automatically surjective, we deduce that (ii) $\Longleftrightarrow$ (iii).

It remains to show that (iii') $\Longleftrightarrow$ (iv). To do this, we start with observing that

$$
\pi^{-1}\left(\operatorname{ker} \beta_{0+}\right)=\pi^{-1}\left(\bigcap_{i=0}^{m^{+}} \operatorname{ker} \beta_{i}\right)=\bigcap_{i=0}^{m^{+}} \pi^{-1}\left(\operatorname{ker} \beta_{i}\right)=\bigcap_{i=0}^{m^{+}}\left(V+\operatorname{ker} B_{i}\right),
$$

where the last equality comes from (8.1). Taking the preimage w.r.t. the surjective map $\pi$ gives that $\operatorname{ker} \beta_{0+}=\{0\}$ is equivalent to $\pi^{-1}\left(\operatorname{ker} \beta_{0+}\right)=V$. The equivalence (iii') $\Longleftrightarrow$ (iv) follows from the above formula.

Eventually, we show that Condition (3.2) is inherited by the maps induced by admissible splits.

Lemma 8.4. Consider a linear subspace $V \subset H$ and the corresponding split. Suppose ker $B_{+} \subseteq$ ker $B_{m+1}$. Then

(i) $\operatorname{ker} b_{+} \subseteq \operatorname{ker} b_{m+1}$,

(ii) if $b_{+}$is surjective then $\operatorname{ker} \beta_{+} \subseteq \operatorname{ker} \beta_{m+1}$.

Proof. (i) This part is obvious since $\operatorname{ker} b_{+}=V \cap \operatorname{ker} B_{+}$and similarly for ker $b_{m+1}$.

(ii) By Lemma 8.1

$$
\pi^{-1}\left(\operatorname{ker} \beta_{m+1}\right)=V+\operatorname{ker} B_{m+1} .
$$

Applying Lemma 8.1 once again, this time to the map $B:=B_{+}$and the subspaces $V \subseteq H$ and $V^{\prime}=B_{+} V \subseteq H^{\prime}:=H_{1} \times \cdots \times H_{m^{+}}$we obtain that the map $\beta: H / V \rightarrow H^{\prime} / V^{\prime}$ being the quotient of $B$ satisfies

$$
\pi^{-1}(\operatorname{ker} \beta)=V+\operatorname{ker} B \text {. }
$$


Since $b_{+}$is surjective, i.e. $V^{\prime}=B_{1} V \times \cdots \times B_{m^{+}} V$, the map $\beta_{+}$coincides with $\varphi \circ \beta$, where $\varphi: H^{\prime} / V^{\prime}=H_{1} \times \cdots \times H_{m^{+}} / B_{1} V \times \cdots \times B_{m^{+}} V \rightarrow H_{1} / B_{1} V \times \cdots \times H_{m^{+}} / B_{m^{+}} V$ is the natural isomorphism, hence $\operatorname{ker} \beta$ and $\operatorname{ker} \beta_{+}$coincide. Therefore,

$$
\pi^{-1}\left(\operatorname{ker} \beta_{+}\right)=V+\operatorname{ker} B_{+}
$$

and using the hypothesis ker $B_{+} \subseteq$ ker $B_{m+1}$ we obtain

$$
\pi^{-1}\left(\operatorname{ker} \beta_{+}\right) \subseteq V+\operatorname{ker} B_{m+1}=\pi^{-1}\left(\operatorname{ker} \beta_{m+1}\right) .
$$

To conclude, it remains to apply the surjective map $\pi$.

Corollary 8.5. Suppose the map $B_{0+}$ is a linear isomorphism and ker $B_{+} \subseteq$ ker $B_{m+1}$. If a split $0 \longrightarrow \mathrm{V} \longrightarrow \mathrm{H} \longrightarrow \mathrm{H} / \mathrm{V} \longrightarrow 0$ is admissible then

(i) $b_{0+}$ is a linear isomorphism and $\operatorname{ker} b_{+} \subseteq \operatorname{ker} b_{m+1}$,

(ii) $\beta_{0+}$ is a linear isomorphism and $\operatorname{ker} \beta_{+} \subseteq \operatorname{ker} \beta_{m+1}$.

Proof. This is a direct consequence of Lemma 8.4 and of the equivalent forms of the admissibility property given in Lemma 8.3 .

8.2. Formulation of the characterization result. Recall $0 \leq m^{+} \leq m$. In addition to the linear surjective maps $B_{k}: H \rightarrow H_{k}, k=0, \ldots, m+1$, we consider real numbers $c_{0}=1, c_{1}, \ldots, c_{m^{+}}>0, c_{m^{+}+1}, \ldots, c_{m} \leq 0$ and $c_{m+1}=-1$. In this context, for any positive definite quadratic forms $\mathcal{Q}_{+}: H_{0} \rightarrow \mathbb{R}$ and $\mathcal{Q}_{-}: H_{m+1} \rightarrow \mathbb{R}$, define a functional $J_{\mathcal{Q}_{+}, \mathcal{Q}_{-}}$acting on non-negative integrable functions $f_{k}: H_{k} \rightarrow \mathbb{R}(k=1, \ldots, m)$ satisfying $\int_{H_{k}} f_{k}>0$ :

$$
J_{\mathcal{Q}_{+}, \mathcal{Q}_{-}}\left(f_{1}, \ldots, f_{m}\right)=\frac{\int_{H} \prod_{k=0}^{m+1} f_{k}^{c_{k}}\left(B_{k} x\right) d x}{\prod_{k=1}^{m}\left(\int_{H_{k}} f_{k}\right)^{c_{k}}},
$$

where

$$
f_{0}=e^{-\mathcal{Q}_{+}} \quad \text { and } \quad f_{m+1}=e^{-\mathcal{Q}_{-}} .
$$

Assuming (3.1) and (3.2), the condition defined below turns out to be equivalent to the positivity of the infimum of $J_{\mathcal{Q}_{+}, \mathcal{Q}_{-}}$over all functions $f_{1}, \ldots, f_{m}$.

Definition 8.6. We say that $H$ together with the maps $B_{k}$ and the exponents $c_{k}(k=$ $0, \ldots, m+1)$ satisfies Condition $(C)$ if for every admissible split

$$
\mathrm{O} \longrightarrow \mathrm{V} \longrightarrow \mathrm{H} \longrightarrow \mathrm{H} / \mathrm{V} \longrightarrow 0,
$$

the following two conditions are satisfied:

(i) if $b_{m+1}$ is a trivial map (i.e. $V \subseteq \operatorname{ker} B_{m+1}$ thus $V_{m+1}=\{0\}$ ) then $V$ is a supercritical subspace of $H$, i.e.

$$
\operatorname{dim} V \geq \sum_{k=1}^{m} c_{k} \operatorname{dim} V_{k}
$$

(ii) if $\beta_{0}$ is a trivial map (i.e. $B_{0} V=B_{0} H$ thus $H_{0} / V_{0}=\{0\}$ ) then $H / V$ is a subcritical quotient of $H$, i.e.

$$
\operatorname{dim} H / V \leq \sum_{k=1}^{m} c_{k} \operatorname{dim} H_{k} / V_{k} .
$$

Later on we will also use a similar notion which we call criticality: 
Definition 8.7. Suppose $V \subset H$ induces an admissible split. We say that $V$ is a critical subspace of $H$ if

$$
b_{m+1} \text { is trivial and } \operatorname{dim} V=\sum_{k=1}^{m} c_{k} \operatorname{dim} V_{k} .
$$

Similarly, we say that $H / V$ is a critical quotient of $H$ if

$$
\beta_{0} \text { is trivial and } \operatorname{dim} H / V=\sum_{k=1}^{m} c_{k} \operatorname{dim} H_{k} / V_{k} .
$$

Theorem 8.8. In the setting described above, suppose that (3.1) and (3.2) hold.

(i) If for some positive definite quadratic forms $\mathcal{Q}_{+}: H_{0} \rightarrow \mathbb{R}$ and $\mathcal{Q}_{-}: H_{m+1} \rightarrow \mathbb{R}$,

$$
\inf _{f_{1}, \ldots, f_{m}} J_{\mathcal{Q}_{+}, \mathcal{Q}_{-}}\left(f_{1}, \ldots, f_{m}\right)>0
$$

then $(H, B, c)$ satisfies Condition $(C)$.

(ii) If $(H, B, c)$ satisfies Condition $(C)$ then for all positive definite quadratic forms $\mathcal{Q}_{+}$and $\mathcal{Q}_{-}$,

$$
\inf _{f_{1}, \ldots, f_{m}} J_{\mathcal{Q}_{+}, \mathcal{Q}_{-}}\left(f_{1}, \ldots, f_{m}\right)>0 .
$$

The above theorem easily implies the characterization of positivity of the functional $J$, for which we present now an intrinsic formulation (in terms of $\mathcal{Q}$ only, and not of its decomposition involving $\left.B_{0}, B_{m+1}\right)$.

Theorem 8.9. Consider the functional $J$ as defined in (1.3) along with a quadratic form $\mathcal{Q}: H \rightarrow \mathbb{R}$, surjective linear maps $B_{k}: H \rightarrow H_{k}(k=1, \ldots, m)$ and exponents $c_{1}, \ldots, c_{m^{+}}>0, c_{m^{+}+1}, \ldots, c_{m} \leq 0$. Suppose the non-degeneracy condition (2.4) and (2.5) hold. Then inf $J>0$ if and only if for every subspace $V \subseteq H$ such that

$$
\operatorname{dim}\left(V \cap\left(\operatorname{ker} B_{+}\right)^{\perp_{\mathcal{Q}}}\right)=\sum_{i=1}^{m^{+}} \operatorname{dim} B_{i} V,
$$

the following two implications hold true:

(i) if $V \subseteq \operatorname{rad} \mathcal{Q}+\operatorname{ker} B_{+}$then

$$
\operatorname{dim} V \geq \sum_{k=1}^{m} c_{k} \operatorname{dim} B_{k} V
$$

(ii) if $V+\left(\operatorname{ker} B_{+}\right)^{\perp_{\mathcal{Q}}}=H$ then

$$
\operatorname{dim} H-\operatorname{dim} V \leq \sum_{k=1}^{m} c_{k}\left(\operatorname{dim} H_{k}-\operatorname{dim} B_{k} V\right) .
$$

Remark 8.10. When no kernel is involved (i.e. $\mathcal{Q}=0$ ) we recover, in a slightly different form, the condition of Theorem 1.5 from the introduction. To see the connection, observe that $(i)$ for $V=H$ and $(i i)$ for $V=\{0\}$ yield $\operatorname{dim} H=\sum_{k=1}^{m} c_{k} \operatorname{dim} H_{k}$. Then, it is clear that the inequalities in $(i)$ and $(i i)$ are equivalent.

Proof of Theorem 8.9. First we construct the maps $B_{0}: H \rightarrow H_{0}$ and $B_{m+1}: H \rightarrow H_{m+1}$ as in the proof of the implication $(1) \Longrightarrow(2)$ from Lemma 3.1. Recall from that proof that

$$
\operatorname{ker} B_{0}=H_{0}^{\perp_{\mathcal{Q}}}=\left(\operatorname{ker} B_{+}\right)^{\perp_{\mathcal{Q}}}
$$

and

$$
\operatorname{ker} B_{m+1}=\operatorname{rad} \mathcal{Q}+\operatorname{ker} B_{+} \text {. }
$$


Next, we apply Theorem 8.8 , and reformulate it in terms of the quadratic form $\mathcal{Q}$ as follows. By (3.1) and Lemma 8.3, a subspace $V \subset H$ is admissible if and only if

$$
\operatorname{dim} V=\sum_{i=0}^{m^{+}} \operatorname{dim} B_{i} V
$$

The last equation is equivalent to (8.4), thanks to the following relation

$$
\operatorname{dim} B_{0} V=\operatorname{dim} V-\operatorname{dim}\left(V \cap \operatorname{ker} B_{0}\right)=\operatorname{dim} V-\operatorname{dim}\left(V \cap\left(\operatorname{ker} B_{+}\right)^{\perp_{\mathcal{Q}}}\right) .
$$

Finally note that the following equivalences hold true:

$$
\begin{aligned}
& V \subseteq \operatorname{ker} B_{m+1} \Longleftrightarrow V \subseteq \operatorname{rad} \mathcal{Q}+\operatorname{ker} B_{+}, \\
& B_{0} V=B_{0} H \quad \Longleftrightarrow \quad V+\operatorname{ker} B_{0}=H \quad \Longleftrightarrow \quad V+\left(\operatorname{ker} B_{+}\right)^{\perp_{\mathcal{Q}}}=H .
\end{aligned}
$$

Eventually, let us note that Lemma 8.3 (i) $\Longleftrightarrow$ (iv) shows that (8.4) is equivalent to

$$
\left(V+\left(\operatorname{ker} B_{+}\right)^{\perp \mathcal{Q}}\right) \cap \bigcap_{i=1}^{m^{+}}\left(V+\operatorname{ker} B_{i}\right)=V .
$$

8.3. Useful notation for the proof of Theorem 8.8. Consider any split

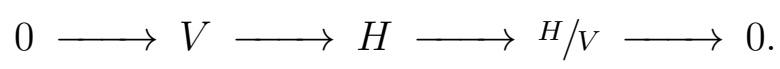

We fix any linear injective maps

$$
\begin{aligned}
& j: H / V \rightarrow H, \\
& j_{k}: H_{k} / V_{k} \rightarrow H_{k} \quad \text { for } k=0, \ldots, m+1
\end{aligned}
$$

such that $j$ (respectively $j_{k}$ ) composed with the canonical quotient map $H \rightarrow{ }^{H} / V$ (resp. $H_{k} \rightarrow H_{k} / V_{k}$ ) is the identity on $H / V$ (resp. $H_{k} / V_{k}$ ). For example, $j$ can be chosen so that its range is the orthogonal complement of $V$ in $H$ (and similarly $j_{k}$ ).

For any $x \in V$ and $y \in H / V$ we write

$$
B_{k}(x+j(y))=B_{k} x+B_{k} j(y)=b_{k} x+\rho_{k} y+j_{k}\left(\beta_{k} y\right),
$$

where

$$
\rho_{k} y=B_{k} j(y)-j_{k}\left(\beta_{k} y\right):{ }^{H} / V \rightarrow V_{k} .
$$

To see that $\rho_{k}$ has range in $V_{k}$, compose it with the quotient map $\pi_{k}: H_{k} \rightarrow H_{k} / V_{k}$ to see that $\pi_{k} \rho_{k}(y)=\pi_{k} B_{k} j(y)-\beta_{k} \pi j(y)=\left(\pi_{k} B_{k}-\beta_{k} \pi\right)(j(y))=0$, by definition of $\beta_{k}$.

Fix any positive definite quadratic forms $\mathcal{Q}_{+}$on $H_{0}$ and $\mathcal{Q}_{-}$on $H_{m+1}$ and thus fix $f_{0}$ and $f_{m+1}$ as in (8.3). Next, let $f_{k}: H_{k} \rightarrow \mathbb{R}(k=1,2, \ldots, m)$ be non-negative, integrable functions with $\int_{H_{k}} f_{k}>0$. By identifying each function $f_{k}: H_{k} \rightarrow \mathbb{R}$ (for $k=0,1, \ldots, m+$ 1) with a function $f_{k}: V_{k} \times H_{k} / V_{k} \rightarrow \mathbb{R}$ and using Fubini theorem we rewrite (8.2) as

$$
J_{\mathcal{Q}_{+}, \mathcal{Q}_{-}}\left(f_{1}, \ldots, f_{m}\right)=C \frac{\int_{H / V} \int_{V} \prod_{k=0}^{m+1} f_{k}^{c_{k}}\left(b_{k} x+\rho_{k} y, \beta_{k} y\right) d x d y}{\prod_{k=1}^{m}\left(\int_{H_{k} / V_{k}} \int_{V_{k}} f_{k}(x, y) d x d y\right)^{c_{k}}},
$$

where $C \in(0,+\infty)$ is a constant resulting from changes of variables $V \times H / V \ni(x, y) \mapsto$ $x+j(y) \in H$ and $V_{k} \times H_{k} / V_{k} \ni(x, y) \mapsto x+j_{k}(y) \in H_{k}$ (for $k=1, \ldots, m$ ). (If $j$ and $j_{k}$ are chosen according to Euclidean structures of $H$ and $H_{k}$ as in the example mentioned above, then $C=1$. However, in what follows the exact value of $C$ has no importance). 
8.4. Necessity of Condition (C). Here we prove the first assertion of Theorem 8.8,

Proof of Theorem 8.8, part (i). Recall the discussion from Section 8.3. Assume that the subspace $V$ is admissible and that we choose the functions $f_{1}, \ldots, f_{m^{+}}$so that they are bounded of compact support and the functions $f_{m^{+}+1}, \ldots, f_{m}$ which are strictly positive with polynomial decay at infinity.

First consider the case $V \subseteq \operatorname{ker} B_{m+1}$ (i.e. $V_{m+1}=\{0\}$ and thus $b_{m+1}$ and $\rho_{m+1}$ are trivial). We aim at showing that $V$ is a supercritical subspace of $H$. To this end, for any $R \in[1, \infty)$, set

$$
f_{k}^{(R)}(x, y)=f_{k}(x / R, y) \quad \text { for } k=1, \ldots, m .
$$

By the hypothesis, for all $R \geq 1, J_{\mathcal{Q}_{+}, \mathcal{Q}_{-}}\left(f_{1}^{(R)}, \ldots, f_{m}^{(R)}\right)$ is uniformly bounded from below by a positive constant. On the other hand, using (8.5) for $\left(f_{1}^{(R)}, \ldots, f_{m}^{(R)}\right)$ and rescaling the variables of integration $x$ in the numerator and the denominator (i.e. replacing $x$ with $R x)$ gives

$$
\begin{aligned}
& J_{\mathcal{Q}_{+}, \mathcal{Q}_{-}}\left(f_{1}^{(R)}, \ldots, f_{m}^{(R)}\right)=C \times R^{\operatorname{dim} V-\sum_{k=1}^{m} c_{k} \operatorname{dim} V_{k}} \\
& \times \frac{\int_{H / V} \int_{V} f_{0}\left(R b_{0} x+\rho_{0} y, \beta_{0} y\right) f_{m+1}^{-1}\left(0, \beta_{m+1} y\right) \prod_{k=1}^{m} f_{k}^{c_{k}}\left(b_{k} x+\frac{1}{R} \rho_{k} y, \beta_{k} y\right) d x d y}{\prod_{k=1}^{m}\left(\int_{H_{k} / V_{k}} \int_{V_{k}} f_{k}(x, y) d x d y\right)^{c_{k}}} .
\end{aligned}
$$

Now it is enough to show that the double integral in the numerator is uniformly bounded from above as $R \rightarrow \infty$. Doing so, the positive lower bound on the l.h.s. of (8.6) implies that $R^{\operatorname{dim} V-\sum_{k=1}^{m} c_{k} \operatorname{dim} V_{k}}$ is bounded away from 0 as $R \rightarrow \infty$ and thus $\operatorname{dim} V-$ $\sum_{k=1}^{m} c_{k} \operatorname{dim} V_{k} \geq 0$.

Due to our choice of the functions $f_{k}$, for $k=1, \ldots, m^{+}$,

$$
\operatorname{supp} f_{k} \subseteq F_{k} \times G_{k}
$$

for some compact, star-shaped sets $F_{k} \subseteq V_{k}$ and $G_{k} \subseteq H_{k} / V_{k}$ (by star-shaped we mean that if $x$ is in a set then so does $\lambda x$ for any $\lambda \in[0,1])$.

Thanks to the assumption (3.1) and the admissibility of the split of $H$, the maps $b_{0+}$ and $\beta_{0+}$ are linear isomorphisms (see Lemma 8.3).

Observe that we can restrict the domain of the outer integral in the numerator of (8.6) to the set

$$
G:=\beta_{+}^{-1}\left(G_{1} \times \cdots \times G_{m^{+}}\right) \subseteq H / V
$$

because outside $G$ the terms $f_{k}^{c_{k}}$ with $k \in\left\{1, \ldots, m^{+}\right\}$make the integrand vanish. Although $G$ is not necessarily compact, this allows us to bound the exponentially large term $f_{m+1}^{-1}$ in (8.6). Indeed, the first assertion of Corollary 8.5)(ii) is

$$
\operatorname{ker} \beta_{+} \subseteq \operatorname{ker} \beta_{m+1},
$$

hence by Lemma 3.3, $\beta_{m+1}(G)$ is a compact subset of $H_{m+1} / V_{m+1}$ and thus we can bound from above the integrand by replacing $f_{m+1}^{-1}$ with

$$
\sigma:=\sup _{\{0\} \times \beta_{m+1}(G)} f_{m+1}^{-1}<\infty .
$$

In order to deal with the terms $f_{k}^{c_{k}}$ for $k \in\left\{m^{+}+1, \ldots, m\right\}$ that grow (at most) polynomially at infinity, we take advantage of the exponential decay of $f_{0}$. In order to use a compactness argument we decompose $f_{0}$ into slices. Namely, note that for some compact, star-shaped sets $F_{0} \subseteq V_{0}, G_{0} \subseteq H_{0} / V_{0}$, which depend on $\mathcal{Q}_{+}$and the map $j_{0}$ only, 
we have

$$
\begin{aligned}
f_{0}\left(x_{0}, y_{0}\right) & =\int_{0}^{1} \mathbf{1}_{\left\{(x, y) \in V_{0} \times H_{0} / V_{0}: \exp \left(-\mathcal{Q}_{+}\left(x+j_{0} y\right)\right) \geq u\right\}}\left(x_{0}, y_{0}\right) d u \\
& =\int_{0}^{\infty} t e^{-t^{2} / 2} \mathbf{1}_{\left\{(x, y) \in V_{0} \times H_{0} / V_{0}: \exp \left(-\mathcal{Q}_{+}\left(x+j_{0} y\right)\right) \geq \exp \left(-t^{2} / 2\right)\right\}}\left(x_{0}, y_{0}\right) d t \\
& =\int_{0}^{\infty} t e^{-t^{2} / 2} \mathbf{1}_{\left\{(x, y) \in V_{0} \times H_{0} / V_{0}: \mathcal{Q}_{+}\left(x+j_{0} y\right) \leq t^{2} / 2\right\}}\left(x_{0}, y_{0}\right) d t \\
& \leq \int_{0}^{\infty} t e^{-t^{2} / 2} \mathbf{1}_{t F_{0}}\left(x_{0}\right) \mathbf{1}_{t G_{0}}\left(y_{0}\right) d t
\end{aligned}
$$

for all $\left(x_{0}, y_{0}\right) \in V_{0} \times H_{0} / V_{0}$. Using Fubini, we can thus bound the numerator of (8.6) by

$$
\sigma \int_{0}^{\infty} t e^{-t^{2} / 2} \int_{H / V} \int_{V} \mathbf{1}_{t F_{0}}\left(R b_{0} x+\rho_{0} y\right) \mathbf{1}_{t G_{0}}\left(\beta_{0} y\right) \prod_{k=1}^{m} f_{k}^{c_{k}}\left(b_{k} x+\frac{1}{R} \rho_{k} y, \beta_{k} y\right) d x d y d t
$$

Now we argue that for some polynomials $p$ and $q$, for any $t>0$ and all $R \geq 1$, the integrand of the double integral w.r.t $x$ and $y$ in (8.7) is bounded from above by $q(t)$ and is supported in a compact set of measure at most $p(t)$.

To this end, fix any $R \geq 1$ and $t>0$. The integrand in question vanishes if $y$ is outside the set $\beta_{0+}^{-1}\left(t G_{0} \times G_{1} \times \cdots \times G_{m^{+}}\right)$. Clearly we have

$$
\beta_{0+}^{-1}\left(t G_{0} \times G_{1} \times \cdots \times G_{m^{+}}\right) \subseteq(t+1) \beta_{0+}^{-1}\left(G_{0} \times G_{1} \times \cdots \times G_{m^{+}}\right) .
$$

Since $\beta_{0+}$ is an isomorphism, the set

$$
\mathbf{G}=\beta_{0+}^{-1}\left(G_{0} \times G_{1} \times \cdots \times G_{m^{+}}\right)
$$

is a compact (and star-shaped) subset of $H / V$. Thus we can restrict the domain of integration w.r.t. $y$ to $(t+1) \mathbf{G}$.

Next, fix $y \in(t+1) \mathbf{G}$ and $R \geq 1$. Take any $x \in V$ such that

$$
\begin{aligned}
& R b_{0} x+\rho_{0} y \in t F_{0}, \\
& b_{k} x+\frac{1}{R} \rho_{k} y \in F_{k} \quad \text { for all } k=1, \ldots, m^{+}
\end{aligned}
$$

(otherwise the integrand is zero). Then we have

$$
\begin{aligned}
& b_{0} x \in \frac{t}{R} F_{0}+\left(-\frac{1}{R} \rho_{0}((t+1) \mathbf{G})\right) \subseteq(t+1)\left(F_{0}+\rho_{0}(-\mathbf{G})\right), \\
& b_{k} x \in F_{k}+\left(-\frac{1}{R} \rho_{k}((t+1) \mathbf{G})\right) \subseteq(t+1)\left(F_{k}+\rho_{k}(-\mathbf{G})\right) \text { for } k=1, \ldots, m^{+},
\end{aligned}
$$

where the inclusion follows from the fact that $F_{0}, F_{1}, \ldots, F_{m^{+}}$and $-\mathbf{G}$ are star-shaped. Consider compact sets

$$
\tilde{F}_{k}=F_{k}+\rho_{k}(-\mathbf{G}) \subseteq V_{k}, \quad \text { for } k=0,1, \ldots, m^{+} .
$$

Put

$$
\mathbf{F}=b_{0+}^{-1}\left(\tilde{F}_{0} \times \tilde{F}_{1} \times \cdots \times \tilde{F}_{m^{+}}\right) .
$$

Clearly $x \in(t+1) \mathbf{F}$ for all $y \in(t+1) \mathbf{G}$ and all $R \geq 1$ and hence one can restrict the integral w.r.t. $x$ to the domain $(t+1) \mathbf{F}$ which is compact, because $b_{0+}$ is an isomorphism. Therefore we have shown that for all $R \geq 1$, the domain of the double integral in (8.7) can be restricted to the compact set $(t+1)(\mathbf{F} \times \mathbf{G})$. Moreover, the measure of this set is a polynomial function of $t$. 
Now we proceed with bounding the integrand inside $(t+1)(\mathbf{F} \times \mathbf{G})$. The functions $f_{1}, \ldots, f_{m^{+}}$are bounded, so we may focus on the terms involving $f_{k}$ for $k \in\left\{m^{+}+1, \ldots m\right\}$. Set

$$
\begin{aligned}
F_{k} & =b_{k}(\mathbf{F})+\rho_{k}(\mathbf{G}), \\
G_{k} & =\beta_{k}(\mathbf{G})
\end{aligned}
$$

for $k=m^{+}+1, \ldots, m$. Then for all $(x, y) \in(t+1)(\mathbf{F} \times \mathbf{G})$, all $R \geq 1$ and $k=$ $m^{+}+1, \ldots, m$

$$
b_{k} x+\frac{1}{R} \rho_{k} y \in b_{k}((t+1) \mathbf{F})+\frac{1}{R} \rho_{k}((t+1) \mathbf{G}) \subseteq(t+1) F_{k}
$$

since $\mathbf{G}$ is star-shaped, and, of course,

$$
\beta_{k} y \in(t+1) G_{k} .
$$

Therefore the integrand can be bounded from above by

$$
\prod_{k=1}^{m^{+}}\left(\sup _{H_{k}} f_{k}\right)^{c_{k}} \times \prod_{k=m^{+}+1}^{m}\left(\sup _{(t+1)\left(F_{k} \times G_{k}\right)} f_{k}^{-1}\right)^{-c_{k}},
$$

where the first product is finite by boundedness of the functions $f_{1}, \ldots, f_{m^{+}}$and the second product is bounded by a polynomial in $t$ due to polynomial decay of the functions $f_{m^{+}+1}, \ldots, f_{m}$. Consequently, (8.6) is upper bounded independently of $R$, as claimed.

Now we pass to the case when $B_{0} V=B_{0} H$ (i.e. $H_{0} / V_{0}=\{0\}$ and thus $\beta_{0}$ is trivial). Using a similar reasoning to the one used in the first case, we will show that $H / V$ is a subcritical quotient of $H$. For any $r \in(0,1]$ set

$$
f_{k}^{(r)}(x, y)=f_{k}(x, y / r) \quad \text { for } k=1, \ldots, m .
$$

We apply (8.5) for $\left(f_{1}^{(r)}, \ldots, f_{m}^{(r)}\right)$ and then we rescale the variables $y$ in the numerator and the denominator (i.e. we replace $y$ with $r y$ in all integrals with respect to $y$ ). We get

$$
\begin{aligned}
& J_{\mathcal{Q}_{+}, \mathcal{Q}_{-}}\left(f_{1}^{(r)}, \ldots, f_{m}^{(r)}\right)=C \times r^{\operatorname{dim} H / V-\sum_{k=1}^{m} c_{k} \operatorname{dim} H_{k} / V_{k}} \\
& \times \frac{\int_{H / V} \int_{V} f_{0}\left(b_{0} x+r \rho_{0} y, 0\right) f_{m+1}^{-1}\left(b_{m+1} x+r \rho_{m+1} y, r \beta_{m+1} y\right) \prod_{k=1}^{m} f_{k}^{c_{k}}\left(b_{k} x+r \rho_{k} y, \beta_{k} y\right) d x d y}{\prod_{k=1}^{m}\left(\int_{H_{k} / V_{k}} \int_{V_{k}} f_{k}(x, y) d x d y\right)^{c_{k}}} .
\end{aligned}
$$

As before, it is enough to show that the double integral in the numerator is uniformly bounded from above as $r \rightarrow 0$.

First we deal with the term $f_{m+1}^{-1}$. The map $\beta_{0+}$ is a linear isomorphism, and since the map $\beta_{0}$ is trivial, also the map $\beta_{+}$is an isomorphism. Therefore, the set

$$
\mathbf{G}:=\beta_{+}^{-1}\left(G_{1} \times \cdots \times G_{m^{+}}\right) \subseteq H / V
$$

to which we can restrict the integral w.r.t. $y$ in (8.9) is compact (and star-shaped). Now, fix any $y \in \mathbf{G}$ and take any $x \in V$ such that $b_{k} x+r \rho_{k} y \in F_{k}$ for all $k=1, \ldots, m^{+}$. Then we have

$$
b_{k} x \in F_{k}+\left(-r \rho_{k}(\mathbf{G})\right) \subseteq F_{k}+\rho_{k}(-\mathbf{G}):=\tilde{F}_{k}
$$

and the sets $\tilde{F}_{k} \subseteq V_{k}$ are compact and star-shaped. Put

$$
F=b_{+}^{-1}\left(\tilde{F}_{1} \times \cdots \times \tilde{F}_{m^{+}}\right) \subseteq V .
$$


The set $F$ is not necessarily compact, but the first assertion of Corollary 8.5 says that ker $b_{+} \subseteq \operatorname{ker} b_{m+1}$ and hence by Lemma 3.3, $b_{m+1}(F)$ is a compact subset of $V_{m+1}$. Therefore for all $r \in(0,1]$ we have the bound

$$
f_{m+1}^{-1}\left(b_{m+1} x+r \rho_{m+1} y, r \beta_{m+1} y\right) \leq \sup _{\left(b_{m+1}(F)+\rho_{m+1}(\mathbf{G})\right) \times \mathbf{G}} f_{m+1}^{-1}<\infty .
$$

In order to deal with the terms $f_{k}^{c_{k}}$ for $k \in\left\{m^{+}+1, \ldots, m\right\}$ we decompose $f_{0}(\cdot, 0)$ into slices. Namely, defining the compact, star-shaped set $F_{0}=\left\{x \in V_{0}: \mathcal{Q}_{+}(x) \leq 1 / 2\right\}$, we have that for all $x_{0} \in V_{0}=H_{0}$,

$$
\begin{aligned}
f_{0}\left(x_{0}, 0\right) & =\int_{0}^{1} \mathbf{1}_{\left\{x \in V_{0}: \exp \left(-\mathcal{Q}_{+}(x)\right) \geq u\right\}}\left(x_{0}\right) d u \\
& =\int_{0}^{\infty} t e^{-t^{2} / 2} \mathbf{1}_{\left\{x \in V_{0}: \exp \left(-\mathcal{Q}_{+}(x)\right) \geq \exp \left(-t^{2} / 2\right)\right\}}\left(x_{0}\right) d t \\
& =\int_{0}^{\infty} t e^{-t^{2} / 2} \mathbf{1}_{t F_{0}}\left(x_{0}\right) d t .
\end{aligned}
$$

Using Fubini, we can thus bound the numerator of (8.9) by a constant (our bound on the terms involving $\left.f_{m+1}^{-1}\right)$ times

$$
\int_{0}^{\infty} t e^{-t^{2} / 2} \int_{H / V} \int_{V} \mathbf{1}_{t F_{0}}\left(b_{0} x+r \rho_{0} y\right) \prod_{k=1}^{m} f_{k}^{c_{k}}\left(b_{k} x+r \rho_{k} y, \beta_{k} y\right) d x d y d t
$$

As discussed above, the domain of the integration w.r.t. $y$ can be restricted to the compact set $\mathbf{G} \subseteq H / V$. Now fix $t>0, y \in \mathbf{G}$ and $r \in(0,1]$. Suppose that $x \in V$ is such that the integrand in (8.10) does not vanish. Then we must have

$$
\begin{aligned}
& b_{0} x+r \rho_{0} y \in t F_{0}, \\
& b_{k} x+r \rho_{k} y \in F_{k} \quad \text { for } k=1, \ldots, m^{+},
\end{aligned}
$$

which implies

$$
\begin{aligned}
& b_{0} x \in t F_{0}+\left(-r \rho_{0}(\mathbf{G})\right) \subseteq(t+1)\left(F_{0}+\rho_{0}(-\mathbf{G})\right)=:(t+1) \tilde{F}_{0}, \\
& b_{k} x \in F_{k}+\left(-r \rho_{k}(\mathbf{G})\right) \subseteq F_{k}+\rho_{k}(-\mathbf{G})=: \tilde{F}_{k} \quad \text { for } k=1, \ldots, m^{+} .
\end{aligned}
$$

Set

$$
\mathbf{F}=b_{0+}^{-1}\left(\tilde{F}_{0} \times \tilde{F}_{1} \times \cdots \times \tilde{F}_{m^{+}}\right) .
$$

Clearly $\mathbf{F}$ is a compact ( $b_{0+}$ is an isomorphism), star-shaped subset of $V$ and $x \in(t+1) \mathbf{F}$.

Finally define the sets $F_{k}$ and $G_{k}$ for $k=m^{+}+1, \ldots, m$ as in (8.8). Then for all $(x, y) \in$ $((t+1) \mathbf{F}) \times \mathbf{G}$ and all $r \in(0,1]$, the arguments of the functions $f_{k}$ for $k=m^{+}+1, \ldots, m$ are in $\left((t+1) F_{k}\right) \times G_{k}$ and therefore the integrand can be bounded from above by

$$
\prod_{k=1}^{m^{+}}\left(\sup _{H_{k}} f_{k}\right)^{c_{k}} \times \prod_{k=m^{+}+1}^{m}\left(\sup _{\left((t+1) F_{k}\right) \times G_{k}} f_{k}^{-1}\right)^{-c_{k}} .
$$

We conclude as in the first case.

8.5. Sufficiency of Condition (C). The inductive proof of the second part of Theorem 8.8 relies on the following lemma. It shows that under (3.1), if one of the components of an admissible split (the subspace or the quotient) is critical, then Condition (C) is inherited by both components of the split. 
Lemma 8.11 (Inheritance of Condition (C) through a critical split). Suppose $H$ together with the maps $B_{k}$ and the exponents $c_{k}$ satisfy Condition $(C)$ and

$$
\mathrm{O} \longrightarrow \mathrm{V} \longrightarrow \mathrm{H} \longrightarrow \mathrm{H} / \mathrm{V} \longrightarrow 0
$$

is an admissible split. If $V$ is a critical subspace of $H$ or $H / V$ is a critical quotient of $H$ then $V$ with the maps $b_{k}$ and the exponents $c_{k}$, as well as $H / V$ with the maps $\beta_{k}$ and the exponents $c_{k}$ satisfy Condition $(C)$.

Proof. First we present the scheme of the proof:

Part $I$. We suppose that $V$ is a critical subspace of $H$.

- Condition (C) for $V$ :

- Subspace of $V$ : supercriticality of a subspace $U$ of $V$ is inherited directly from supercriticality of $U$ as a subspace of $H$.

- Quotient of $V$ : subcriticality of the quotient $V / U$ of $V$ follows from criticality of $V$ in $H$ and supercriticality of $U$ in $V$ just proved.

- Condition (C) for $H / V$ :

- Subspace of $H / V$ : supercriticality of a subspace $U$ of $H / V$ follows from supercriticality of a subspace $\tilde{U}=\pi^{-1}(U)$ (where $\pi: H \rightarrow H / V$ is a natural quotient map) in $H$ and criticality of $V$ in $H$.

- Quotient of $H / V$ : subcriticality of the quotient ${ }^{H / V} / U$ of $H / V$ follows from subcriticality of the quotient $H / \tilde{U}$ of $H$.

Part II. We suppose that $H / V$ is a critical quotient of $H$. After dualizing, i.e. interchanging subspaces with quotient and supercriticality with subcriticality, the arguments are analogous to the ones from Part I.

- Condition (C) for $H / V$ :

- Quotient of $H / V$ : subcriticality of a quotient ${ }^{H / V} / U$ of $H / V$ is inherited directly from subcriticality of $H / \tilde{U}$ as a quotient of $H$, where $\tilde{U}=\pi^{-1}(U)$.

- Subspace of $H / V$ : supercriticality of the subspace $U$ of $H / V$ follows from criticality of $H / V$ in $H$ and subcriticality of $H / V / U$ in $H / V$ just proved.

- Condition (C) for $V$ :

- Quotient of $V$ : subcriticality of a quotient $V / U$ of $V$ follows from subcriticality of a quotient $H / U$ in $H$ and criticality of $H / V$ in $H$.

- Subspace of $V$ : supercriticality of the subspace $U$ of $V$ follows from supercriticality of the subspace $U$ of $H$.

Next we give the arguments in details. In the first part we assume that $V$ is a critical subspace: $V \subseteq$ ker $B_{m+1}$, and thus the map $b_{m+1}$ is trivial, and the following equality holds

$$
\operatorname{dim} V=\sum_{k=1}^{m} c_{k} \operatorname{dim} B_{k} V .
$$

Let us check Condition (C) for $V$ equipped with the maps $\left(b_{k}\right)$ and the coefficients $\left(c_{k}\right)$ (for shortness, we will write $(V, b)$, since the coefficients $c$ are the same for all sub-structures).

Suppose that

$$
0 \longrightarrow U \longrightarrow V \longrightarrow V / U \longrightarrow 0
$$

is an admissible split of $(V, b)$. Since $U \subseteq V=\operatorname{ker} b_{m+1}$, we must check supercriticality of $U$ as a subspace of $(V, b)$. The admissible split of $(V, b)$ induced by $U$ obviously leads to an admissible split of $(H, B)$. Moreover, $U \subseteq V \subseteq$ ker $B_{m+1}$, so that Condition (C) for $(H, B)$ yields

$$
\operatorname{dim} U \geq \sum_{k=1}^{m} c_{k} \operatorname{dim} B_{k} U
$$


Using $B_{i} U=b_{i} U$, we conclude that $U$ is a supercritical subspace of $(V, b)$.

For the same $U, V / U$ is a subcritical quotient of $(V, b)$ (even regardless whether $b_{0}(U)=$ $b_{0}(V)$ or not) because $V$ is a critical subspace of $H$ and $U$ is a supercritical subspace of $V$ (subtract the inequality $\operatorname{dim} U \geq \sum_{k=1}^{m} c_{k} \operatorname{dim} b_{k} U$ from (8.11)).

Now we check Condition (C) for $(H / V, \beta)$. Suppose

$$
\mathrm{O} \longrightarrow \mathrm{U} \longrightarrow \mathrm{H} / \mathrm{V} \longrightarrow \mathrm{H} / \mathrm{V} / \mathrm{U} \longrightarrow 0
$$

is an admissible split of $(H / V, \beta)$, which by Lemma 8.3 (i) $\Longleftrightarrow$ (iv) means that

$$
\bigcap_{i=0}^{m^{+}}\left(U+\operatorname{ker} \beta_{i}\right)=U \text {. }
$$

Taking the preimage w.r.t. $\pi: H \rightarrow{ }^{H} / V$ we get

$$
\bigcap_{i=0}^{m^{+}}\left(\pi^{-1}(U)+V+\operatorname{ker} B_{i}\right)=\pi^{-1}(U),
$$

where we used (8.1) and the relation $\pi^{-1}(A+B)=\pi^{-1}(A)+\pi^{-1}(B)+\operatorname{ker} \pi$ which is valid for any linear surjective map. Denote $\tilde{U}=\pi^{-1}(U)$. Of course $\tilde{U}$ contains $V$, hence the above assertion means that

$$
0 \longrightarrow \tilde{U} \longrightarrow H \longrightarrow H / \tilde{U} \longrightarrow 0
$$

is an admissible split of $(H, B)$ (again use Lemma 8.3 (i) $\Longleftrightarrow$ (iv)).

We need to check supercriticality of $U$ as a subspace of $H / V$ whenever $U \subseteq \operatorname{ker} \beta_{m+1}$. By criticality of $V$ in $H$ we have $V \subseteq \operatorname{ker} B_{m+1}$, which combined with the assertion $U \subseteq \operatorname{ker} \beta_{m+1}$ and (8.1) yields

$$
\tilde{U}=\pi^{-1}(U) \subseteq \pi^{-1}\left(\operatorname{ker} \beta_{m+1}\right)=V+\operatorname{ker} B_{m+1}=\operatorname{ker} B_{m+1} .
$$

Applying Condition $(\mathrm{C})$ for $(H, B)$, we know that:

$$
\operatorname{dim} \tilde{U} \geq \sum_{k=1}^{m} c_{k} \operatorname{dim} B_{k} \tilde{U} .
$$

If we subtract from the last inequality the relationship (8.11) corresponding to criticality of $V$ in $(H, B)$, we get the supercriticality of $U$ in $(H / V, \beta)$. Indeed, up to isomorphism

$$
U \approx \tilde{U} / V \text { and } \beta_{k} U \approx B_{k} \tilde{U} / B_{k} V
$$

as one readily checks by considering the ranges and kernels of the maps $\pi: \tilde{U} \rightarrow U$ and $\phi: B_{k} \tilde{U} \rightarrow B_{k} H / B_{k} V$ defined by $\phi(x)=x+B_{k} V$.

Now we check subcriticality of the quotient ${ }^{H / V} / U$ of $H / V$ whenever $\beta_{0}(U)=\beta_{0}(H / V)$, i.e. $U+\operatorname{ker} \beta_{0}=H / V$. Notice that using (8.1), we have

$$
H=\pi^{-1}\left(U+\operatorname{ker} \beta_{0}\right)=\tilde{U}+V+\operatorname{ker} B_{0}=\tilde{U}+\operatorname{ker} B_{0}
$$

(the last equality follows from the fact that $V \subseteq \tilde{U})$, that is $B_{0}(\tilde{U})=B_{0}(H)$. Therefore, by Condition $(\mathrm{C})$ for $(H, B)$, the quotient $H / \tilde{U}$ must be subcritical in $(H, B)$, that is

$$
\operatorname{dim} H / \tilde{U} \leq \sum_{k=1}^{m} c_{k} \operatorname{dim} B_{k} H / B_{k} \tilde{U} .
$$

Using again (8.12) together with the relation $\beta_{k} H / V=B_{k} H / B_{k} V$, we may rewrite the above inequality as

$$
\operatorname{dim}{ }^{H / V} / U \leq \sum_{k=1}^{m} c_{k} \operatorname{dim} \beta_{k}{ }^{H} / V / \beta_{k} U
$$


In other words, ${ }^{H / V} / U$ is a subcritical quotient of $(H / V, \beta)$.

In the second part, suppose $H / V$ is a critical quotient of $(H, B)$. More specifically, $B_{0}(V)=B_{0}(H)$, i.e. $\beta_{0}$ is trivial, and

$$
\operatorname{dim} H / V=\sum_{k=1}^{m} c_{k} \operatorname{dim} B_{k} H / B_{k} V .
$$

The reasoning below is analogous to the first part after interchanging subspaces with quotient and supercriticality with subcriticality.

We check Condition (C) for $(H / V, \beta)$. Consider a split of $H / V$ via its subspace $U$ and suppose this split is admissible. We need to check subcriticality of ${ }^{H} / \mathrm{V} / \mathrm{U}$ in $H / V$ whatever $U$ is, because $\beta_{0}$ is trivial and so $\beta_{0}(U)=\beta_{0}(H / V)$ always holds. To this end, consider a split of $H$ via $\tilde{U}=\pi^{-1}(U)$, which is admissible, as we already showed. Moreover,

$$
B_{0}(\tilde{U}) \supseteq B_{0}(V)=B_{0}(H),
$$

so we can use subcriticality of $H / \tilde{U}$ in $(H, B)$. From the latter, subcriticality of ${ }^{H / V} / U$ in $(H / V, \beta)$ follows, as we have already explained.

For the same $U, U$ is a supercritical subspace of $H / V$ (regardless whether $U \subseteq$ ker $\beta_{m+1}$ or not), because $H / V$ is a critical quotient of $H$ and ${ }^{H / V} / U$ is a subcriticial quotient of $H / V$ (write the corresponding equality and inequality relations and subtract them).

Next, we check Condition (C) for $(V, b)$. Consider a split of $V$ via a subspace $U$ of $V$ which is admissible. We use an induced split of $H$ via $U$, which is also admissible.

We need to check subcriticality of $V / U$ in $V$ whenever $b_{0}(U)=b_{0}(V)$. Since we know that

$$
B_{0}(U)=b_{0}(U)=b_{0}(V)=B_{0}(V)=B_{0}(H)
$$

(the last equality is due to criticality of $H / V$ in $H$ ), we can use subcriticality of $H / U$ in $H$. Writing the corresponding inequality for dimensions and subtracting from it the equality (8.11) related to criticality of $H / V$ in $(H, B)$, we get subcriticality of $V / U$ in $(V, b)$.

Finally we check supercriticality of $U$ in $(V, b)$ whenever $U \subseteq \operatorname{ker} b_{m+1}$. Since the latter implies $U \subseteq \operatorname{ker} B_{m+1}$, we can invoke the fact that $U$ is a supercritical subspace of $H$ to conclude.

Our next result is about tensorization through a split. We say that $(H, B, c)=$ $\left(H,\left(B_{k}\right)_{k=0}^{m+1},\left(c_{k}\right)_{k=1}^{m}\right)$ admits the strong positivity property if for every positive definite quadratic forms $\mathcal{Q}_{+}$on $H_{0}$ and $\mathcal{Q}_{-}$on $H_{m+1}$,

$$
\inf _{\left(f_{1}, \ldots, f_{m}\right)} \frac{\int_{H} e^{-\mathcal{Q}_{+}\left(B_{0} x\right)+\mathcal{Q}_{-}\left(B_{m+1} x\right)} \prod_{k=1}^{m} f_{k}^{c_{k}}\left(B_{k} x\right) d x}{\prod_{k=1}^{m}\left(\int_{H_{k}} f_{k}\right)^{c_{k}}}>0 .
$$

Lemma 8.12 (Tensorization). Assume that (H,B) satisfies (3.1) and (3.2). Let $V$ be a linear subspace of $H$ which induces an admissible split.

If $(V, b, c)$ and $(H / V, \beta, c)$ have the strong positivity property, then $(H, B, c)$ has it too.

Proof. Fix any non-negative, integrable functions $f_{k}: H_{k} \rightarrow \mathbb{R}(k=1, \ldots, m)$ satisfying $\int_{H_{k}} f_{k}>0$. Set $f_{0}=e^{-\mathcal{Q}_{+}}, f_{m+1}=e^{-\mathcal{Q}_{-}}, c_{0}=1$ and $c_{m+1}=-1$. Our goal is to bound from below the quantity $J_{\mathcal{Q}_{+}, \mathcal{Q}_{-}}\left(f_{1}, \ldots, f_{m}\right)$ defined in (8.2) by a positive constant (not depending on $\left(f_{1}, \ldots, f_{m}\right)$ ). Recall the discussion from Section 8.3 and in particular Formula (8.5). Our aim is to bound from below the quantity

$$
I:=\int_{H / V} \int_{V} \prod_{k=0}^{m+1} f_{k}^{c_{k}}\left(b_{k} x+\rho_{k} y, \beta_{k} y\right) d x d y
$$


by application of an inequality of inverse Brascamp-Lieb type on $V$ and on $H / V$.

By Corollary [8.5, $b_{0+}=\left(b_{0}, b_{+}\right)$is surjective and ker $b_{+} \subseteq \operatorname{ker} b_{m+1}$. Hence, by Lemma 2.6

$$
V=\operatorname{ker} b_{0}+\operatorname{ker} b_{+} \subseteq \operatorname{ker} b_{0}+\operatorname{ker} b_{m+1} \subseteq V .
$$

Using Lemma 2.6 once again, we obtain that $\left(b_{0}, b_{m+1}\right)$ is surjective. This allows us to remove the cross-terms from the Gaussian kernel: indeed for every $y \in{ }^{H} / V$, there exists $v_{y} \in V$ such that $b_{0} v_{y}=\rho_{0} y$ and $b_{m+1} v_{y}=\rho_{m+1} y$. Using the translation invariance of the Lebesgue measure on $V$, we apply the change of variable $V \ni x \mapsto x-v_{y} \in V$ to the inner integral of (8.14), and get that it is equal to

$$
\int_{H / V} \int_{V} f_{0}\left(b_{0} x, \beta_{0} y\right) f_{m+1}^{-1}\left(b_{m+1} x, \beta_{m+1} y\right) \prod_{k=1}^{m} f_{k}^{c_{k}}\left(b_{k} x-b_{k} v_{y}+\rho_{k} y, \beta_{k} y\right) d x d y .
$$

Next, we bound from below the Gaussian kernel by a product kernel. Since $f_{0}=$ $\exp \left(-\mathcal{Q}_{+}\right)$where $\mathcal{Q}_{+}$is viewed as a quadratic form on $V_{0} \times H_{0} / V_{0}$, we can bound $f_{0}$ from below by

$$
f_{0}\left(x_{0}, y_{0}\right) \geq f_{0, V}\left(x_{0}\right) f_{0, H / V}\left(y_{0}\right),
$$

where $f_{0, V}=\exp \left(-\mathcal{Q}_{+, V}\right)$ and $f_{0, H / V}=\exp \left(-\mathcal{Q}_{+, H / V}\right)$ for some positive definite quadratic forms $\mathcal{Q}_{+, V}: V_{0} \rightarrow \mathbb{R}$ and $\mathcal{Q}_{+, H_{0} / V_{0}}: H / V \rightarrow \mathbb{R}$.

For $f_{m+1}$ we use a reverse bound, namely for some positive definite quadratic forms $\mathcal{Q}_{-, V}: V_{m+1} \rightarrow \mathbb{R}$ and $\mathcal{Q}_{-, H_{m+1} / V_{m+1}}: H / V \rightarrow \mathbb{R}$ we have

$$
f_{m+1}^{-1}\left(x_{0}, y_{0}\right) \geq f_{m+1, V}^{-1}\left(x_{0}\right) f_{m+1, H / V}^{-1}\left(y_{0}\right),
$$

where $f_{m+1, V}=\exp \left(-\mathcal{Q}_{-, V}\right)$ and $f_{m+1, H / V}=\exp \left(-\mathcal{Q}_{-, H / V}\right)$. Observe that we have used here the fact that $\mathcal{Q}_{-}$is positive definite. We get that $I$ from (8.14) is at least

$\int_{H / V} f_{0, H / V}\left(\beta_{0} y\right) f_{m+1, H / V}^{-1}\left(\beta_{m+1} y\right) \int_{V} f_{0, V}\left(b_{0} x\right) f_{m+1, V}^{-1}\left(b_{m+1} x\right) \prod_{k=1}^{m} f_{k}^{c_{k}}\left(b_{k} x-b_{k} v_{y}+\rho_{k} y, \beta_{k} y\right) d x d y$.

By the strong positivity property for $(V, b, c)$ there exists a constant $C_{V}>0$ such that for all $y \in H / V$,

$$
\begin{aligned}
\int_{V} f_{0, V}\left(b_{0} x\right) & f_{m+1, V}^{-1}\left(b_{m+1} x\right) \prod_{k=1}^{m} f_{k}^{c_{k}}\left(b_{k} x-b_{k} v_{y}+\rho_{k} y, \beta_{k} y\right) d x \\
& \geq C_{V} \prod_{k=1}^{m}\left(\int_{V_{k}} f_{k}\left(\cdot-b_{k} v_{y}+\rho_{k} y, \beta_{k} y\right)\right)^{c_{k}} \\
& =C_{V} \prod_{k=1}^{m}\left(\int_{V_{k}} f_{k}\left(\cdot, \beta_{k} y\right)\right)^{c_{k}},
\end{aligned}
$$

where the equality follows from translation invariance of the Lebesgue measure on each $V_{k}$. Denoting $f_{k, H / V}(y):=\int_{V_{k}} f_{k}(\cdot, y)$ for $y \in H_{k} / V_{k}(k=1, \ldots, m)$, we obtain

$$
I \geq C_{V} \int_{H / V} f_{0, H / V}\left(\beta_{0} y\right) f_{m+1, H / V}^{-1}\left(\beta_{m+1} y\right) \prod_{k=1}^{m} f_{k, H / V}^{c_{k}}\left(\beta_{k} y\right) d y .
$$

Now it remains to apply the strong positivity property for $(H / V, \beta, c)$ and the functions $f_{k, H / V}$ in order to get

$$
I \geq C_{V} C_{H / V} \prod_{k=1}^{m}\left(\int_{H_{k} / V_{k}} f_{k, H / V}\right)^{c_{k}}=C_{V} C_{H / V} \prod_{k=1}^{m}\left(\int_{H_{k} / V_{k}} \int_{V_{k}} f_{k}(x, y) d x d y\right)^{c_{k}}
$$

for some constant $C_{H / V}>0$ (which depends on $\mathcal{Q}_{+, H / V}$ and $\mathcal{Q}_{-, H / V}$ ). 
Proposition 8.13. Let $0 \leq m^{+} \leq m$ be integers and consider surjective maps $B_{k}: H \rightarrow$ $H_{k}$ for $k=0,1, \ldots, m+1$ and real numbers $c_{k}$ such that $c_{k}>0$ for $k=1, \ldots, m^{+}$and $c_{k} \leq 0$ for $k=m^{+}+1, \ldots, m$. Assume that Conditions (3.1) and $(C)$ hold. Then

- for all $i=1, \ldots, m^{+}, \operatorname{dim} H_{i}>0 \Longrightarrow c_{i} \geq 1$

- if $H$ is a critical subspace, then $B_{0}=B_{m+1}=0$.

Proof. Fix $1 \leq i \leq m^{+}$such that $\operatorname{dim} H_{i}>0$, i.e. $\operatorname{ker} B_{i} \neq H$. Consider $V=\operatorname{ker} B_{i}$ and a related split of $H$ by $V$. Since clearly

$$
\bigcap_{k=0}^{m^{+}}\left(V+\operatorname{ker} B_{k}\right)=V
$$

by Lemma 8.3 , the split is admissible. Moreover, since $B_{0+}$ is surjective, the map $\left(B_{0}, B_{i}\right)$ is surjective too, hence Lemma 2.6 yields

$$
H=\operatorname{ker} B_{0}+\operatorname{ker} B_{i}=\operatorname{ker} B_{0}+V
$$

i.e. $B_{0} V=B_{0} H$. Therefore we can use the fact that the quotient $H / V$ of $H$ is subcritical, from which it follows that

$$
\operatorname{dim} H / V \leq \sum_{k=1}^{m} c_{k} \operatorname{dim} H_{k} / V_{k} \leq \sum_{k=1}^{m^{+}} c_{k} \operatorname{dim} H_{k} / V_{k} .
$$

For all $1 \leq k \leq m^{+}$with $k \neq i$, the map $\left(B_{i}, B_{k}\right)$ is surjective, hence again by Lemma 2.6.

$$
H=\operatorname{ker} B_{i}+\operatorname{ker} B_{k}=V+\operatorname{ker} B_{k},
$$

which means that $B_{k} V=B_{k} H$, i.e. $H_{k} / V_{k}=\{0\}$. Thus (8.17) boils down to

$$
\operatorname{dim} H / V \leq c_{i} \operatorname{dim} H_{i} / V_{i} .
$$

Recall that $V_{i}=B_{i} V$ is reduced to $\{0\}$ since by definition $V=\operatorname{ker} B_{i}$. Moreover

$$
\operatorname{dim} H / V=\operatorname{dim} H-\operatorname{dim} \operatorname{ker} B_{i}=\operatorname{dim} B_{i} H=\operatorname{dim} H_{i},
$$

so the last inequality can be rewritten as $\operatorname{dim} H_{i} \leq c_{i} \operatorname{dim} H_{i}$. Therefore $c_{i} \geq 1$ if $\operatorname{dim} H_{i}>$ 0

The proof of the second item follows the same lines. Firstly, $H$ is admissible by hypothesis. Since it is assumed to be a critical subspace, we know that $H \subset$ ker $B_{m+1}$ hence $B_{m+1}=0$, and that

$$
\operatorname{dim} H=\sum_{k=1}^{m} c_{k} \operatorname{dim} B_{k} H .
$$

We set $V=\operatorname{ker} B_{0}$. As above, we can check that $V$ is admissible. Since $V \subset H=$ ker $B_{m+1}$, it is a supercritical subspace thanks to Condition (C). Therefore, using the above dimension equality, we get after subtraction

$$
\operatorname{dim} H / V \leq \sum_{k=1}^{m} c_{k} \operatorname{dim} B_{k} H / B_{k} V \leq \sum_{k=1}^{m^{+}} c_{k} \operatorname{dim} B_{k} H / B_{k} V
$$

again. Since $B_{0+}$ is a bijection and $V$ is admissible, we know by Lemma 8.3 that $\operatorname{dim} H=\sum_{i=0}^{m^{+}} \operatorname{dim} B_{i} H$ and $\operatorname{dim} V=\sum_{i=0}^{m^{+}} \operatorname{dim} B_{i} V$. Hence $\operatorname{dim} H / V=\sum_{i=0}^{m^{+}} \operatorname{dim} B_{i} H / B_{i} V$. Plugging this equality into (8.18) yields after rearranging

$$
\operatorname{dim} B_{0} H / B_{0} V \leq \sum_{i=1}^{m^{+}}\left(c_{i}-1\right) \operatorname{dim} B_{i} H / B_{i} V .
$$


Since $B_{0+}$ is surjective, the map $\left(B_{0}, B_{i}\right)$ is surjective too for any $1 \leq i \leq m^{+}$. Hence Lemma 2.6 yields $H=\operatorname{ker} B_{0}+\operatorname{ker} B_{i}=V+\operatorname{ker} B_{i}$, which ensures that $B_{i} H=B_{i} V$. Therefore, (8.19) becomes $\operatorname{dim} B_{0} H / B_{0} V \leq 0$. Recall that by definition $B_{0} V=\{0\}$. We can conclude that $\operatorname{dim} B_{0} H=0$, that is $B_{0}=0$.

The next statements will help to initialize the inductive proof of Theorem 8.8 (ii).

Lemma 8.14. Assertion (ii) of Theorem 8.8 is true when $\operatorname{dim} H=1$.

Proof. The main tool here is the reverse Hölder inequality for several functions: Let $c_{1} \geq 0 \geq c_{2}, \ldots c_{m}$ with $\sum_{k} c_{k}=1$ then

$$
\int_{\mathbb{R}^{d}} \prod_{k} f_{k}^{c_{k}} \geq \prod_{k}\left(\int_{\mathbb{R}^{d}} f_{k}\right)^{c_{k}}
$$

holds for all integrable non-negative functions with $\int_{\mathbb{R}^{d}} f_{k} \in(0,+\infty)$. This inequality follows from its version for two functions applied with $\lambda=c_{1} \geq 1$ :

$$
\int_{\mathbb{R}^{d}} \prod_{k} f_{k}^{c_{k}} \geq\left(\int f_{1}\right)^{c_{1}}\left(\int \prod_{j=2}^{m} f_{j}^{\frac{c_{j}}{c_{2}+\cdots+c_{m}}}\right)^{c_{2}+\cdots+c_{m}},
$$

and from the classical Hölder inequality applied to the second integral (observe that the inner exponents sum up to 1 and are all non-negative, while the outer exponent $c_{2}+\cdots+c_{m}$ is non-positive).

Since $\operatorname{dim} H=1$ and for $1 \leq k \leq m, B_{k}: H \rightarrow H_{k}$ is surjective and $H_{k}$ is non-trivial, it follows that the maps $B_{k}, k \geq 1$ are bijections. Therefore we may reduce to the case $H=H_{k}=\mathbb{R}$ and $B_{k}=$ Id for $1 \leq k \leq m$. In this simple setting, the only possible subspaces $V$ are 0 and $\mathbb{R}$. The former is trivially admissible, while the latter is admissible by hypothesis. Hence Condition $(\mathrm{C})$ rewrites as:

- if $\mathbb{R} \subset \operatorname{ker} B_{m+1}$ (i.e. $B_{m+1}=0$ ), then $1 \geq \sum_{k=1}^{m} c_{k}$,

- if $B_{0}\{0\}=B_{0} \mathbb{R}$ (i.e. $B_{0}=0$ ) then $1 \leq \sum_{k=1}^{m} c_{k}$,

Also the hypothesis of bijectivity of $\left(B_{0}, B_{+}\right)$reduces to two cases: either $B_{0}=0, B_{+}=B_{1}$ and $c_{1} \geq 0 \geq c_{2}, \ldots, c_{m}$, or $B_{0} \neq 0, B_{+}=0$ and $0 \geq c_{1}, \ldots, c_{m}$.

In order to prove the lemma, we consider several cases:

Case 1: If $B_{0}=B_{m+1}=0$, then Condition (C) rewrites as $\sum_{k=1}^{m} c_{k}=1$. Moreover there is no kernel and, as explained above $c_{1} \geq 0 \geq c_{2}, \ldots, c_{m}$. The positivity of the Brascamp-Lieb functional is a direct consequence of the reverse Hölder inequality (8.20).

Case 2: if $B_{0} \neq 0$ and $B_{m+1}=0$, then $0 \geq c_{1}, \ldots, c_{m}$ and Condition (C) amounts to $1 \geq \sum_{k=1}^{m} c_{k}$. We define $c_{0}:=1-\sum_{k=1}^{m} c_{k} \geq 1$ and we are ready to apply the inverse Hölder inequality with $m+1$ functions:

$$
\int e^{-\mathcal{Q}_{+}\left(B_{0} x\right)} \prod_{j=1}^{m} f_{j}(x)^{c_{j}} d x \geq\left(\int e^{-\frac{1}{c_{0}} \mathcal{Q}_{+}\left(B_{0} x\right)}\right)^{c_{0}} \prod_{j=1}^{m}\left(\int f_{j}\right)^{c_{j}} .
$$

Case 3: if $B_{0}=0$ and $B_{m+1} \neq 0$, then $c_{1} \geq 0 \geq c_{2}, \ldots, c_{m}$ and Condition (C) reads as $1 \leq \sum_{k=1}^{m} c_{k}$ (actually the inequality is strict. If it where an equality then $H=\mathbb{R}$ would be a critical space, which is not compatible with $B_{m+1} \neq 0$ as explained by Proposition 8.13). We define $c_{m+1}:=1-\sum_{k=1}^{m} c_{k}<0$ and we apply the inverse Hölder inequality with $m+1$ functions:

$$
\int e^{\mathcal{Q}_{-}\left(B_{m+1} x\right)} \prod_{k=1}^{m} f_{k}(x)^{c_{k}} d x \geq\left(\int e^{\frac{1}{c_{m+1}} \mathcal{Q}_{-}\left(B_{0} x\right)}\right)^{c_{m+1}} \prod_{k=1}^{m}\left(\int f_{k}\right)^{c_{k}} .
$$


Since $c_{m+1}<0$ and $\mathcal{Q}_{-}$is positive definite, the first integral of the right-hand side term is finite.

Case 4: $B_{0} \neq 0$ and $B_{m+1} \neq 0$ does not happen. Indeed it implies that $B_{+}=0$ but then the condition $\operatorname{ker} B_{+} \subset \operatorname{ker} B_{m+1}$ is violated. A more conceptual explanation is that a quadratic form on $\mathbb{R}$ is either zero, definite positive or definite negative, so that the above three cases cover all possibilities.

Lemma 8.15. Assertion (ii) of Theorem 8.8 is true when $m=2$ and $B_{0}=B_{m+1}=0$.

Proof. Our goal is to prove the positivity of the Brascamp-Lieb functional for two functions and no kernel. Our hypothesis is that $B_{+}$is bijective and that Condition (C) holds. Since $1 \leq m^{+} \leq m=2$ we can consider two cases:

Case 1: $m=m^{+}=2$. Proposition 8.13 yields $c_{1}, c_{2} \geq 1$. Condition (C) ensures that $H$ is a critical space, hence

$$
\operatorname{dim} H=c_{1} \operatorname{dim} H_{1}+c_{2} \operatorname{dim} H_{2} \geq \operatorname{dim} H_{1}+\operatorname{dim} H_{2}=\operatorname{dim} H,
$$

where the latter inequality comes from the fact that $B_{+}=\left(B_{1}, B_{2}\right): H \rightarrow H_{1} \times H_{2}$ is a linear isomorphism. The intermediate inequality cannot be strict, therefore $c_{1}=c_{2}=1$. The inverse Brascamp-Lieb inequality in this case follows from Fubini theorem, after changing variables:

$$
\begin{aligned}
\int_{H} f_{1}\left(B_{1} x\right) f_{2}\left(B_{2} x\right) d x & =\left|\operatorname{det}\left(\left(B_{1}, B_{2}\right)\right)\right|^{-1} \int_{H_{1} \times H_{2}} f_{1}(y) f_{2}(z) d y d z \\
& =\left|\operatorname{det}\left(\left(B_{1}, B_{2}\right)\right)\right|^{-1} \int_{H_{1}} f_{1} \int_{H_{2}} f_{2} .
\end{aligned}
$$

Case 2: $m^{+}=1$ and therefore $B_{1}$ is bijective. Any linear subspace is admissible in this case $\left(\operatorname{dim} V=\operatorname{dim} B_{1} V\right)$. Thus, for any subspace $V$, Condition $(\mathrm{C})$ yields

$$
\operatorname{dim} V \geq c_{1} \operatorname{dim} B_{1} V+c_{2} \operatorname{dim} B_{2} V=c_{1} \operatorname{dim} V+c_{2} \operatorname{dim} B_{2} V
$$

and after rearranging the terms

$$
\left(c_{1}-1\right) \operatorname{dim} V \leq\left|c_{2}\right| \operatorname{dim} B_{2} V .
$$

Choosing $V=\operatorname{ker} B_{2}$, we get that $\left(c_{1}-1\right) \operatorname{dim}$ ker $B_{2}=0$.

Subcase 1: If ker $B_{2}=0$, then $B_{2}$ is an isomorphism. Since $B_{1}$ is also an isomorphism, the relation $\operatorname{dim} H=c_{1} \operatorname{dim} H_{1}+c_{2} \operatorname{dim} H_{2}$ implies $c_{1}+c_{2}=1$. Recall that $c_{1} \geq 0 \geq c_{2}$. We can conclude with the inverse Hölder inequality:

$$
\begin{aligned}
\int f_{1}\left(B_{1} x\right)^{c_{1}} f_{2}\left(B_{2} x\right)^{c_{2}} d x & \geq\left(\int f_{1}\left(B_{1} x\right) d x\right)^{c_{1}}\left(\int f_{2}\left(B_{2} x\right) d x\right)^{c_{2}} \\
& =\left(\left|\operatorname{det} B_{1}\right|^{-1} \int_{H_{1}} f_{1}\right)^{c_{1}}\left(\left|\operatorname{det} B_{2}\right|^{-1} \int_{H_{2}} f_{2}\right)^{c_{2}} .
\end{aligned}
$$

Subcase 2: $c_{1}=1$. Using also that $\operatorname{dim} H=\operatorname{dim} H_{1}$, the equality $\operatorname{dim} H=c_{1} \operatorname{dim} H_{1}+$ $c_{2} \operatorname{dim} H_{2}$ implies that $c_{2} \operatorname{dim} H_{2}=0$, hence $c_{2}=0$. The inverse Brascamp-Lieb inequality is trivial in this case: $\int f_{1}\left(B_{1} x\right) d x=\left|\operatorname{det} B_{1}\right|^{-1} \int f_{1}$.

Lemma 8.16. Assertion (ii) of Theorem 8.8 is true when $m=0$ and when $m=1$.

Proof. Since $0 \leq m^{+} \leq m \leq 1$, we consider three cases.

Case 0: when $m^{+}=m=0$, i.e. there are no functions $f_{k}$. By hypothesis $\mathcal{Q}$ is positive definite. Condition (C) is empty. The conclusion holds as $\int e^{-\mathcal{Q}}>0$. 
Case 1: when $m^{+}=0, c_{1} \leq 0, B_{+}=0$ and by hypothesis $B_{0}$ is a linear isomorphism. Moreover the condition $\operatorname{ker} B_{+} \subset \operatorname{ker} B_{m+1}$ implies that $B_{m+1}=0$. In this setting, Condition (C) is empty. Indeed the inequality $\operatorname{dim} V \geq c_{1} \operatorname{dim} B_{1} V$ is valid for every subspace since $c_{1} \leq 0$. In addition, $B_{0}$ being and isomorphism, the only subspace $V$ such that $B_{0} V=B_{0} H$ is $H$ (and the quotient dimension condition is empty). Consequently, our task is to show that

$$
\inf _{f_{1}} \frac{\int_{H} e^{-\mathcal{Q}_{+}\left(B_{0} x\right)} f_{1}\left(B_{1} x\right)^{c_{1}} d x}{\left(\int_{H_{1}} f_{1}\right)^{c_{1}}}>0 .
$$

The case $c_{1}=0$ is obvious since $\mathcal{Q}_{+} \circ B_{0}$ is positive definite. Next, we assume that $c_{1}<0$. By definition $B_{1}: H \rightarrow H_{1}$ is surjective. We complete it to a bijective map $\Phi: H \rightarrow H_{1} \times \tilde{H}$ of the form $\Phi(x)=\left(B_{1}(x), \tilde{B}(x)\right)$. Using the bijective change of variables $x=\Phi^{-1}(y, \tilde{y})$, there exits $\alpha \in(0,+\infty)$ such that for any $f_{1}$,

$$
\int_{H} e^{-\mathcal{Q}_{+}\left(B_{0} x\right)} f_{1}\left(B_{1} x\right)^{c_{1}} d x=\alpha \int_{H_{1} \times \tilde{H}} e^{-\mathcal{Q}_{+} \circ B_{0} \circ \Phi^{-1}(y, \tilde{y})} f_{1}(y)^{c_{1}} d y d \tilde{y} .
$$

There exists positive definite quadratic forms $\mathcal{Q}_{1}$ on $H_{1}$ and $\tilde{\mathcal{Q}}$ on $\tilde{H}$ such that for all $(y, \tilde{y})$,

$$
\mathcal{Q}_{+} \circ B_{0} \circ \Phi^{-1}(y, \tilde{y}) \leq \mathcal{Q}_{1}(y)+\tilde{\mathcal{Q}}(\tilde{y})
$$

Therefore the latter integral is at most

$\int_{\tilde{H}} e^{-\tilde{\mathcal{Q}}(\tilde{y})} d \tilde{y} \int_{H_{1}} e^{-\mathcal{Q}_{1}(y)} f^{c_{1}}(y) d y \geq\left(\int_{\tilde{H}} e^{-\tilde{\mathcal{Q}}(\tilde{y})} d \tilde{y}\right) \times\left(\int_{H_{1}} e^{-\frac{1}{1-c_{1}} \mathcal{Q}_{1}(y)} d y\right)^{1-c_{1}}\left(\int_{H_{1}} f_{1}\right)^{c_{1}}$, where the latter inequality is a consequence of the inverse Hölder inequality.

Case 2: $m^{+}=m=1$. In this case $c_{1} \geq 0,\left(B_{0}, B_{1}\right)$ is bijective and ker $B_{+}=\operatorname{ker} B_{1} \subset$ ker $B_{m+1}$. We may assume that ker $B_{1} \neq H$, otherwise $H_{1}=\{0\}$ and we can discard the function $f_{1}$ and we are back to Case 0 .

Condition (C) asserts that every admissible subspace $V$ verifies:

- if $V \subset \operatorname{ker} B_{m+1}$ then $\operatorname{dim} V \geq c_{1} \operatorname{dim} B_{1} V$

- if $B_{0} V=B_{0} H$ then $\operatorname{dim}^{H} / V \leq c_{1} \operatorname{dim}{ }^{B_{1} H} / B_{1} V$

Observe that the latter "quotient condition" boils down to $c_{1} \geq 1$ : Indeed by hypothesis $\operatorname{dim} H=\operatorname{dim} B_{0} H+\operatorname{dim} B_{1} H$, and $V$ is admissible if and only if $\operatorname{dim} V=\operatorname{dim} B_{0} V+$ $\operatorname{dim} B_{1} V$. Therefore, when $V$ also satisfies that $B_{0} V=B_{0} H$, taking the difference of the latter two dimension equalities yields $\operatorname{dim} H / V=\operatorname{dim} B_{1} H / B_{1} V$. Hence the condition $\operatorname{dim} H / V \leq c_{1} \operatorname{dim} B_{1} H / B_{1} V$ becomes $\left(c_{1}-1\right) \operatorname{dim} H / V \geq 0$ which can be an empty condition (when $H=V$ ) or equivalent to $c_{1} \geq 1 \mathrm{e}$.g. for $V=\operatorname{ker} B_{1}$ (see the argument of Proposition 8.13 ).

Given $\left(B_{0}, B_{1}, B_{m+1}=B_{2}\right)$, the set $\mathcal{C}_{1}$ of indices $c_{1} \geq 0$ satisfying Condition $(\mathrm{C})$ is clearly a closed convex subset of $[1,+\infty)$. Indeed, it is defined by the inequality $c_{1} \geq 1$ and conditions of the form $\operatorname{dim} V \geq c_{1} \operatorname{dim} B_{1} V$ (and there are finitely many of them since the dimensions are bounded). Obviously $1 \in \mathcal{C}_{1}$. For $c_{1}=1$, the corresponding Brascamp-Lieb inequality holds with a positive constant. Indeed for every non-negative function $f_{1}$,

$$
\begin{aligned}
\int_{H} e^{-\mathcal{Q}_{+}\left(B_{0} x\right)+\mathcal{Q}_{-}\left(B_{2} x\right)} f_{1}\left(B_{1} x\right) d x & \geq \int_{H} e^{-\mathcal{Q}_{+}\left(B_{0} x\right)} f_{1}\left(B_{1} x\right) d x \\
& =\left|\operatorname{det}\left(\left(B_{0}, B_{1}\right)\right)\right|^{-1} \int_{H_{0}} e^{-\mathcal{Q}_{+}} \int_{H_{1}} f_{1},
\end{aligned}
$$

where we have used the bijection $\left(B_{0}, B_{1}\right)$ in order to change variables. 
Consider the subspace $V=\operatorname{ker} B_{0} \cap \operatorname{ker} B_{m+1}$. Using our hypothesis ker $B_{1} \subset \operatorname{ker} B_{m+1}$, we get that

$$
V \subset\left(V+\operatorname{ker} B_{0}\right) \cap\left(V+\operatorname{ker} B_{1}\right) \subset \operatorname{ker} B_{0} \cap \operatorname{ker} B_{m+1}=V .
$$

Hence, by (8.3), $V \subset \operatorname{ker} B_{m+1}$ is admissible. Consequently $\operatorname{dim} V=\operatorname{dim} B_{0} V+\operatorname{dim} B_{1} V=$ $\operatorname{dim} B_{1} V$ and any $c_{1} \in \mathcal{C}_{1}$ verifies $\operatorname{dim} V \geq c_{1} \operatorname{dim} B_{1} V$ which can be rewritten as $0 \geq$ $\left(c_{1}-1\right) \operatorname{dim} B_{1} V$.

Subcase 1: if $B_{1} V \neq\{0\}$ then the latter inequality implies that $c_{1} \leq 1$. We have shown that $\mathcal{C}_{1}=\{1\}$ and we have established a non-trivial inverse Brascamp-Lieb inequality for $c_{1}=1$.

Subcase 2: if $B_{1} V=\{0\}$. This condition can be rephrased as $V \subset$ ker $B_{1}$. From this, we deduce that

$$
V=\operatorname{ker} B_{0} \cap \operatorname{ker} B_{m+1} \subset \operatorname{ker} B_{0} \cap \operatorname{ker} B_{1}=\{0\},
$$

where the last equality comes from the injectivity of $\left(B_{0}, B_{1}\right)$. The latter is actually bijective so that

$$
\text { ker } B_{0} \oplus \operatorname{ker} B_{1}=H \text {. }
$$

Since $\operatorname{ker} B_{1} \subset \operatorname{ker} B_{m+1}$, and $V=\operatorname{ker} B_{0} \cap \operatorname{ker} B_{m+1}=\{0\}$, we also have

$$
\operatorname{ker} B_{0} \oplus \operatorname{ker} B_{m+1}=H \text {. }
$$

The previous two decompositions of $H$ into direct sums, and the inclusion ker $B_{1} \subset$ ker $B_{m+1}$ imply that ker $B_{1}=\operatorname{ker} B_{m+1}$. Because of this equality, the subspace constraint in Condition (C) is empty: indeed, if $V \subset \operatorname{ker} B_{m+1}=\operatorname{ker} B_{1}$ then $B_{1} V=0$ and $\operatorname{dim} V \geq$ $c_{1} \operatorname{dim} B_{1} V=0$ is true. Therefore the set of numbers $c_{1}$ verifying Condition (C) is $[1,+\infty)$ and our task is to prove a non-trivial inverse Brascamp-Lieb inequality for all exponents $c_{1} \geq 1$. We have already dealt with $c_{1}=1$, so we may restrict our attention to $c_{1}>1$. Observe that the equality ker $B_{1}=\operatorname{ker} B_{m+1}$ ensures the existence of a linear isomorphism $\Psi: H_{1} \rightarrow H_{m+1}$ such that $B_{m+1}=\Psi \circ B_{1}$. Thus for every non-negative function $f_{1}$ :

$$
\begin{aligned}
& \int_{H} e^{-\mathcal{Q}_{+}\left(B_{0} x\right)-\mathcal{Q}_{-}\left(B_{m+1} x\right)} f_{1}^{c_{1}}\left(B_{1} x\right) d x \\
& \quad=\int_{H} e^{-\mathcal{Q}_{+}\left(B_{0} x\right)} e^{\mathcal{Q}_{-} \circ \Psi\left(B_{1} x\right)} f_{1}^{c_{1}}\left(B_{1} x\right) d x \\
& \quad=\left|\operatorname{det}\left(\left(B_{0}, B_{1}\right)\right)\right|^{-1} \int_{H_{0}} e^{-\mathcal{Q}_{+}(y)} \int_{H_{1}} e^{\mathcal{Q}_{-} \circ \Psi(z)} f_{1}^{c_{1}}(z) d z \\
& \geq\left|\operatorname{det}\left(\left(B_{0}, B_{1}\right)\right)\right|^{-1} \int_{H_{0}} e^{-\mathcal{Q}_{+}(y)}\left(\int_{H_{1}} e^{\frac{1}{1-c_{1}} \mathcal{Q}_{-} \circ \Psi(z)} d z\right)^{1-c_{1}}\left(\int_{H_{1}} f_{1}\right)^{c_{1}},
\end{aligned}
$$

where we have used the change of variables $(y, z)=\left(B_{0} x, B_{1} x\right)$ and the inverse Hölder inequality. The proof is complete.

Proof of Theorem 8.8 (ii). First of all, we can assume $\operatorname{dim} H_{k} \geq 1$ for all $k \in\{1, \ldots, m\}$, otherwise one can reduce the problem by discarding all functions $f_{k}$ for which $\operatorname{dim} H_{k}=0$ while Condition $(\mathrm{C})$ and the strong positivity property related to the reduced problem remain equivalent to those related to the original problem.

Let $D_{1}:=[1,+\infty)^{m^{+}} \times(-\infty, 0]^{m-m^{+}} \subseteq \mathbb{R}^{m}$. Our main interest is in the following set:

$$
\begin{aligned}
\mathcal{C}: & =\left\{c \in(0,+\infty)^{m^{+}} \times(-\infty, 0]^{m-m^{+}} ;(H, B, c) \text { satisfies Condition }(\mathrm{C})\right\} \\
& =\left\{c \in D_{1} ;(H, B, c) \text { satisfies Condition }(\mathrm{C})\right\},
\end{aligned}
$$

where the latter equality comes from Proposition 8.13, Since Condition (C) means that the vector $c$ verifies several closed linear inequalities, the second expression of $\mathcal{C}$ proves 
that it is closed and convex. More specifically, the triplet $(H, B, c)$ satisfies Condition $(\mathrm{C})$ if and only if $c$ belongs to all sets in the following two families:

- For any non-trivial subspace $V \subseteq H$ which induces an admissible split and satisfies $V \subseteq \operatorname{ker} B_{m+1}$ consider

$$
S_{V}=\left\{x \in \mathbb{R}^{m}: \sum_{k=1}^{m} x_{k} \operatorname{dim} B_{k} V \leq \operatorname{dim} V\right\} .
$$

Typically, $S_{V}$ is a closed half-space of $\mathbb{R}^{m}$, but it can happen that $S_{V}$ is the whole $\mathbb{R}^{m}$.

- Similarly, for any proper subspace $V \subsetneq H$ which induces an admissible split and satisfies $B_{0} V=B_{0} H$ consider

$$
S^{H / V}=\left\{x \in \mathbb{R}^{m}: \sum_{k=1}^{m} x_{k} \operatorname{dim} B_{k} H / B_{k} V \geq \operatorname{dim} H / V\right\} .
$$

The set $S^{H / V}$ is always a half-space of $\mathbb{R}^{m}$, since at least for some $1 \leq k \leq m^{+}$, $B_{k} V \neq B_{k} H$ (otherwise for each $k=0,1, \ldots, m^{+}, B_{k} V=B_{k} H$, i.e. $V+\operatorname{ker} B_{k}=$ $H$, which by Lemma 8.3 would contradict admissibility of the split).

Even though there are infinitely many subspaces $V$, the coefficients $\operatorname{dim} B_{k} V$ and $\operatorname{dim} B_{k} H / B_{k} V$ take finitely many values. Hence there are finitely many different half-spaces in the above families, and the set $\mathcal{C}$ is a closed convex polyhedron (which may be unbounded).

Since $\mathcal{C}$ is closed, it follows from its original definition that its boundary is covered by the union of the affine hyperplanes in the following three families:

$\mathcal{P}=\left\{\partial S_{V}:\{0\} \neq V \subsetneq H\right.$ induces an admissible split and $\left.V \subseteq \operatorname{ker} B_{m+1}\right\}$

$\cup\left\{\partial S^{H / V}:\{0\} \neq V \subsetneq H\right.$ induces an admissible split and $\left.B_{0} V=B_{0} H\right\}$, $\mathcal{P}_{0}=\left\{\partial S_{H}\right\}$ if $B_{m+1}=0$ or $B_{0}=0$ (note that $\partial S_{H}=\partial S^{H}$ ) otherwise $\mathcal{P}_{0}=\emptyset$ and

$$
\mathcal{B}=\left\{\left\{x \in \mathbb{R}^{m}: x_{k}=0\right\}: k=m^{+}+1, \ldots, m\right\} .
$$

Our aim is to show that $c \in \mathcal{C}$ (i.e. Condition $(\mathrm{C})$ ) implies the strong positivity property for $(H, B, c)$. We proceed by induction in $(\operatorname{dim} H, m)$ with a partial order on $(n, m) \in \mathbb{Z}_{+}^{2}$ given by $\left(n_{1}, m_{1}\right) \preceq\left(n_{2}, m_{2}\right)$ if and only if $n_{1} \leq n_{2}$ and $m_{1} \leq m_{2}$. The founding cases are $m \in\{0,1\}$ with any $\operatorname{dim} H$ (this is treated in Lemma 8.16) and $\operatorname{dim} H=1$ with any $m \geq 1$ (see Lemma 8.14).

The induction step, in which $\operatorname{dim} H \geq 2$ and $m \geq 2$, goes as follows. Take any vector $p=\left(p_{1}, \ldots, p_{m}\right) \in \mathbb{R}^{m}$ such that $p_{1}, \ldots, p_{m^{+}}>0$ and $p_{m^{+}+1}, \ldots, p_{m}<0$ and let $b \in \mathbb{R}$ be a constant such that the affine hyperplane

$$
P=\left\{x \in \mathbb{R}^{m}: \sum_{k=1}^{m} p_{k} x_{k}=b\right\}
$$

contains $c$. Then $D_{1} \cap P$ is a compact subset of $\mathbb{R}^{m}$ and thus $\mathcal{C}_{P}:=\mathcal{C} \cap P$ is a compact convex set (actually it is a compact convex polytope). In what follows, we restrict our considerations to the hyperplane $P$.

Due the convexity property established in Proposition 6.1, it is enough to prove the strong positivity property for the vectors of exponents being vertices of $\mathcal{C}_{P}$. Therefore assume $c$ is a vertex of $\mathcal{C}_{P}$. Consequently, $c$ belongs to an intersection of $P$ and some $m-1$ distinct affine hyperplanes from the family $\mathcal{B} \cup \mathcal{P} \cup \mathcal{P}_{0}$. Consider three cases:

Case 1. Among these $m-1$ affine hyperplanes there is at least one which belongs to $\mathcal{B}$. This means that for some $k \in\left\{m^{+}+1, \ldots, m\right\}, c_{k}=0$. Then we can discard the function $f_{k}$ and in this way reduce the number of functions considered from 
$m$ to $m-1$. Since neither Condition (C) nor the strong positivity property is affected by this reduction (both assertions remain equivalent for the original and the reduced problem), we are done by the induction hypothesis.

Case 2. Among these $m-1$ affine hyperplanes there is at least one which belongs to $\mathcal{P}$. Hence for some $\{0\} \neq V \subsetneq H, V$ is a critical subspace or $H / V$ is a critical quotient (this may happen only when $\operatorname{dim} H \geq 2$ ). Since both $V$ and $H / V$ have dimension strictly smaller than $\operatorname{dim} H$ and thanks to Lemma 8.11, Condition (C) is satisfied for $(V, b, c)$ and $(H / V, \beta, c)$, we can apply the induction hypothesis and get the strong positivity property for $(V, b, c)$ and $(H / V, \beta, c)$. Now the strong positivity property for $(H, B, c)$ follows from Lemma 8.12 .

Case 3. Neither Case 1 nor Case 2 holds, i.e. all $m-1$ distinct affine hyperplanes are in $\mathcal{P}_{0}$. This is possible only when $m=2$ and $\mathcal{P}_{0}=\left\{\partial S_{H}\right\}$, i.e. $B_{0}$ or $B_{m+1}$ is trivial. Then $c \in \partial S_{H} \cap P$. If $B_{m+1}$ is trivial then $H$ is a critical subspace and Proposition 8.13 implies that $B_{0}$ is also trivial and we can conclude using Lemma 8.15. If $B_{0}$ is trivial then ${ }^{H} /\{0\}$ is a critical quotient, i.e.

$$
\operatorname{dim} H=\sum_{k=1}^{m} c_{k} \operatorname{dim} B_{k} H .
$$

Condition (C) tells us that for every subspace $V \subseteq H$ which induces an admissible split, the quotient $H / V$ is subcritical. Subtracting the corresponding inequality from (8.21) gives that $V$ satisfies

$$
\operatorname{dim} V \geq \sum_{k=1}^{m} c_{k} \operatorname{dim} B_{k} V,
$$

regardless whether $V \subseteq$ ker $B_{m+1}$ or not. Therefore Condition (C) for our problem implies Condition $(\mathrm{C})$ which corresponds to the problem with the same vector of exponents $c$ and the same maps $B_{0}, \ldots, B_{m}$ and with $B_{m+1}=0$. Lemma 8.15 ensures the strong positivity property for the modified problem which in turn clearly implies the strong positivity property for the original problem.

\section{REFERENCES}

[1] S. Alesker, S. Dar, and V. Milman, A remarkable measure preserving diffeomorphism between two convex bodies in $\mathbf{R}^{n}$, Geom. Dedicata 74 (1999), no. 2, 201-212. MR 1674116 (2000a:52004) 24, 25

[2] R. D. Anderson and V. L. Klee, Jr., Convex functions and upper semi-continuous collections, Duke Math. J. 19 (1952), 349-357. MR 004734623

[3] Keith Ball, Volumes of sections of cubes and related problems, Geometric aspects of functional analysis (1987-88), Lecture Notes in Math., vol. 1376, Springer, Berlin, 1989, pp. 251-260. MR 1008726 (90i:52019) 2

[4] - Volume ratios and a reverse isoperimetric inequality, J. London Math. Soc. (2) 44 (1991), no. 2, 351-359. MR 1136445 (92j:52013) 2

[5] Convex geometry and functional analysis, Handbook of the geometry of Banach spaces, Vol. I, North-Holland, Amsterdam, 2001, pp. 161-194. MR 18636922

[6] F. Barthe, Inégalités fonctionnelles et géométriques obtenues par transport des mesures, $\mathrm{Ph} . \mathrm{D}$. thesis, Université de Marne-la-Vallée, 1997.4

[7] F. Barthe, Optimal Young's inequality and its converse: a simple proof, Geom. Funct. Anal. 8 (1998), no. 2, 234-242. MR 1616143 (99f:42021) [5

[8] F. Barthe and D. Cordero-Erausquin, Inverse Brascamp-Lieb inequalities along the heat equation, Geometric aspects of functional analysis, Lecture Notes in Math., vol. 1850, Springer, Berlin, 2004, pp. 65-71. MR 20871515

[9] Franck Barthe, Inégalités de Brascamp-Lieb et convexité, C. R. Acad. Sci. Paris Sér. I Math. 324 (1997), no. 8, 885-888. MR 1450443 (98a:26022) 4 
[10] _ An extremal property of the mean width of the simplex, Math. Ann. 310 (1998), no. 4, 685-693. MR 1619740 (99b:52006) 4

[11] _ On a reverse form of the Brascamp-Lieb inequality, Invent. Math. 134 (1998), no. 2, 335361. MR 1650312 (99i:26021) 3, 4, 5, 6, 21, 34, 40

[12] Franck Barthe and Paweł Wolff, Positivity improvement and Gaussian kernels, C. R. Math. Acad. Sci. Paris 352 (2014), no. 12, 1017-1021. MR 32768124 5

[13] William Beckner, Inequalities in Fourier analysis, Ann. of Math. (2) 102 (1975), no. 1, 159-182. MR 0385456 (52 \#6317) 2

[14] Jonathan Bennett and Neal Bez, Closure properties of solutions to heat inequalities, J. Geom. Anal. 19 (2009), no. 3, 584-600. MR 24965675

[15] Jonathan Bennett, Anthony Carbery, Michael Christ, and Terence Tao, The Brascamp-Lieb inequalities: finiteness, structure and extremals, Geom. Funct. Anal. 17 (2008), no. 5, 1343-1415. MR 2377493 (2009c:42052) 5, 6, 36, 42

[16] __ Finite bounds for Hölder-Brascamp-Lieb multilinear inequalities, Math. Res. Lett. 17 (2010), no. 4, 647-666. MR 2661170 6, 42

[17] Rajendra Bhatia, Matrix analysis, Graduate Texts in Mathematics, vol. 169, Springer-Verlag, New York, 1997. MR 1477662 (98i:15003) 34

[18] Christer Borell, Positivity improving operators and hypercontractivity, Math. Z. 180 (1982), no. 2, 225-234. MR 661699 (84b:47029) 3, 15

[19] _ Diffusion equations and geometric inequalities, Potential Anal. 12 (2000), no. 1, 49-71. MR 1745333 囵

[20] Herm Jan Brascamp and Elliott H. Lieb, Best constants in Young's inequality, its converse, and its generalization to more than three functions, Advances in Math. 20 (1976), no. 2, 151-173.

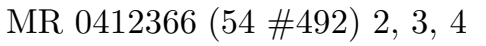

[21] Yann Brenier, Décomposition polaire et réarrangement monotone des champs de vecteurs, C. R. Acad. Sci. Paris Sér. I Math. 305 (1987), no. 19, 805-808. MR 92320322

[22] L. A. Caffarelli, A localization property of viscosity solutions to the Monge-Ampère equation and their strict convexity, Ann. of Math. (2) 131 (1990), no. 1, 129-134. MR 103835924

[23] Luis A. Caffarelli, The regularity of mappings with a convex potential, J. Amer. Math. Soc. 5 (1992), no. 1, 99-104. MR 1124980 (92j:35018) 24

[24] E. A. Carlen, E. H. Lieb, and M. Loss, A sharp analog of Young's inequality on $S^{N}$ and related entropy inequalities, J. Geom. Anal. 14 (2004), no. 3, 487-520. MR 2077162 5 , 6]

[25] Wei-Kuo Chen, Nikos Dafnis, and Grigoris Paouris, Improved Hölder and reverse Hölder inequalities for Gaussian random vectors, Adv. Math. 280 (2015), 643-689. MR 3350230 4, 5, 38, 39, 40

[26] Herbert Federer, Geometric measure theory, Die Grundlehren der mathematischen Wissenschaften, Band 153, Springer-Verlag New York Inc., New York, 1969. MR 0257325 28

[27] Helmut Finner, A generalization of Hölder's inequality and some probability inequalities, Ann. Probab. 20 (1992), no. 4, 1893-1901. MR 11880472

[28] Cristian E. Gutiérrez, The Monge-Ampère equation, Progress in Nonlinear Differential Equations and their Applications, vol. 44, Birkhäuser Boston, Inc., Boston, MA, 2001. MR 182916224

[29] Roger A. Horn and Charles R. Johnson, Matrix analysis, 2 ed., Cambridge University Press, 2012. 33, 36.

[30] Michel Ledoux, Remarks on Gaussian noise stability, Brascamp-Lieb and Slepian inequalities, Geometric aspects of functional analysis, Lecture Notes in Math., vol. 2116, Springer, Cham, 2014, pp. 309-333. MR 3364694 5

[31] Joseph Lehec, Short probabilistic proof of the Brascamp-Lieb and Barthe theorems, Canad. Math. Bull. 57 (2014), no. 3, 585-597. MR 3239122 [5

[32] L. Leindler, On a certain converse of Hölder's inequality. II, Acta Sci. Math. (Szeged) 33 (1972), no. 3-4, 217-223. MR 21993723

[33] Elliott H. Lieb, Gaussian kernels have only Gaussian maximizers, Invent. Math. 102 (1990), no. 1, 179-208. MR 1069246 (91i:42014) 1 2

[34] L. H. Loomis and H. Whitney, An inequality related to the isoperimetric inequality, Bull. Amer. Math. Soc 55 (1949), 961-962. MR 0031538 (11,166d) 2

[35] Robert J. McCann, Existence and uniqueness of monotone measure-preserving maps, Duke Math. J. 80 (1995), no. 2, 309-323. MR 136939522

[36] Joe Neeman, A multidimensional version of noise stability, Electron. Commun. Probab. 19 (2014), no. 72,10 . MR 3274518 囵 
[37] Edward Nelson, The free Markoff field, J. Functional Analysis 12 (1973), 211-227. MR MR0343816 (49 \#8556) 2

[38] András Prékopa, On logarithmic concave measures and functions, Acta Sci. Math. (Szeged) 34 (1973), 335-343. MR 04045573

[39] R. Tyrrell Rockafellar, Convex analysis, Princeton Mathematical Series, No. 28, Princeton University Press, Princeton, N.J., 1970. MR 0274683 22, 23, 24, 25, 30

[40] Alexander Schrijver, Combinatorial optimization. Polyhedra and efficiency. Vol. A, Algorithms and Combinatorics, vol. 24, Springer-Verlag, Berlin, 2003, Paths, flows, matchings, Chapters 1-38. MR 195692447

[41] Stefán Ingi Valdimarsson, On the Hessian of the optimal transport potential, Ann. Sc. Norm. Super.

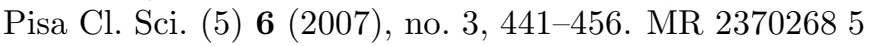

[42] Cédric Villani, Topics in optimal transportation, Graduate Studies in Mathematics, vol. 58, American Mathematical Society, Providence, RI, 2003. MR 1964483 (2004e:90003) 23, 24

(F. Barthe and P. Wolff) Institut de Mathématiques de Toulouse; UMR 5219, Université DE Toulouse; CNRS, France

E-mail address, F. Barthe: franck.barthe@math.univ-toulouse.fr

E-mail address, P. Wolff: pwolff@mimuw.edu.pl 